\title{
Total Synthesis of Papulacandin D
}

Scott E. Denmark, Christopher S. Regens and Tetsuya Kobasyashi Department of Chemistry, University of Illinois Urbana-Champaign, Urbana, Illinois 61801

\section{SUPPORTING INFORMATION}

Table of Contents

Page

General Experimental

Literature Preparations

Experimental Procedures

Preparation of Aromatic Iodide 4

Preparation of Spirocyclic Arylglycoside 20

Preparation of Acid 2

Acylation and Global Deprotection

${ }^{1} \mathrm{H}$ and ${ }^{13} \mathrm{C}$ NMR Spectra

\section{General Experimental}

All reactions were performed in oven $\left(120^{\circ} \mathrm{C}\right)$ and/or flame dried glassware under an atmosphere of dry nitrogen or argon, unless noted. Syringes and needles were dried $\left(120^{\circ} \mathrm{C}\right)$ for at least 12 hours. All reaction temperatures correspond to internal temperatures measured by Teflon-coated thermocouples unless otherwise noted. Reaction solvents including dichloromethane (Fisher, HPLC Grade), diethyl ether (Fisher, BHT stabilized HPLC Grade) and tetrahydrofuran (Fisher, HPLC Grade), toluene (Fisher, ACS Grade) were dried by percolation through a column packed with neutral alumina and a column packed with Q5 reactant, a supported copper catalyst for scavenging oxygen, under a positive pressure of argon. Reaction solvent acetonitrile (Fisher, HPLC grade) was distilled from sodium, $N, N$-dimethylformamide (Aldrich, ACS Grade) and dimethyl sulfoxide (Fisher, ACS Grade) were distilled from $\mathrm{CaH}_{2}$ and dried sequentially over two batches of activated $4 \AA$ molecular sieves. Benzene (Aldrich ACS Grade) was distilled from sodium and benzophenone, dichloroethane (Aldrich ACS Grade) was distilled from $\mathrm{CaH}_{2}$, methanol (Aldrich ACS Grade) was distilled from $\mathrm{MgOMe}$ and 
chloroform (Aldrich ACS Grade) was distilled from $\mathrm{P}_{2} \mathrm{O}_{5}$ and deacidfied by percolating through basic Bockmann Act I grade alumina and stored over freshly activated $3 \AA$ sieves. Pyridine (Aldrich ACS Grade) and 2,6-lutidine (Aldrich ACS Grade) were freshly distilled from $\mathrm{CaH}_{2}$ prior to use. Acetone (Fisher, ACS Grade) was used without further purification.

Solvents for chromatography were: hexanes (Fisher, ACS Grade), ethyl acetate (Aldrich, ACS Grade), diethyl ether (Fisher, ACS Grade), dichloromethane (Aldrich, ACS Grade), toluene (Aldrich, ACS Grade), methanol (Aldrich ACS Grade) and chloroform (Aldrich ACS Grade).

Analytical thin-layer chromatography was preformed on Merck silica or aluminum oxide, basic gel plates with QF-254 indicator. Visualization was accomplished with UV (254 nm), iodine, potassium permanganate $\left(\mathrm{KMnO}_{4}\right)$, vanillin solution, ceric ammonium molybdate (CAM), $p$-anisaldehyde staining solutions.

Column chromatography was performed using Silicycle Silaflash P60 (40-63 $\mu, 60 \AA$ pore size) silica gel. Geraniol (Aldrich) was enriched by spinning band distillation prior to use. 3,5-Dihydroxyrobenzoic acid (Aldrich) was recrystallized from $\mathrm{H}_{2} \mathrm{O}$. Tris(dibenzylideneacetone)-dipalladium chloroform $\left(\mathrm{Pd}_{2}(\mathrm{dba})_{3} \cdot \mathrm{CHCl}_{3}\right)$ was prepared by recrystallization of $\mathrm{Pd}_{2}(\mathrm{dba})_{3}$ from chloroform. ${ }^{1,2}$ Triethylsilyl chloride (TESCl, Gelest), 2(Trimethylsilyl)ethanol (TMS ethanol) (Gelest) were freshly distilled prior to use. 4Dimethylaminopyridine (DMAP) (Aldrich) was recrystallized from ethyl acetate prior to use. Diisopropylethylamine ( $i$ - $\left.\mathrm{Pr}_{2} \mathrm{NEt}\right)$ (Aldrich) and triethylamine $\left(\mathrm{Et}_{3} \mathrm{~N}\right)$ (Aldrich) were freshly distilled from $\mathrm{CaH}_{2}$ prior to use. 2,4,6-Trichlorobenzoyl chloride (Aldrich), acrolein (Fluka) were distilled prior to use

(1,3-Bis-(2,4,6-trimethylphenyl)-2-imidazolidinylidene)dichloro(phenylmethylene)(tricyclohexylphosphine)ruthenium (Grubb's $2^{\text {nd }}$ Generation Catalysts) (Materia), di-tertbutylchlorosilane (Gelest), pivalolyl chloride (Fluka, 99\%+), $N$-iodosuccinimide (NIS) (Aldrich, 95\%), dimethyl sulfide (DMS) (Aldrich, 99\%+), citric acid monohydrate (Fisher), lithium hydroxide monohydrate (Fisher), sodium thiosulfate pentahydrate (Aldrich, ACS Grade), pyridium- $p$-toluenesulfonate (PPTS) (Avocado, 98\%), $p$-toluenesulfonyl chloride (TsCl) $(99 \%+$, Acros), di-chlorobis[( $p$-cymene)chlororuthenium(II)] (Strem, 98\%), triphosgene (TCI, 98\%), ethanol (absolute, Aaper), potassium trimethylsilanoate (TMSOK) (Gelest, 95\%), sodium tertbutoxide ( $\mathrm{NaO} t-\mathrm{Bu}$ ) (Strem, 97\%), hydrofluoric acid (HF) (Fisher, 49\%), thionyl chloride $\left(\mathrm{SOCl}_{2}\right)$ (Aldrich, 97\%), and $(S)-(+)-$ and $(R)-(-)-\alpha$-methoxy- $\alpha$-trifluoromethylphenylacetyl 
chloride (MPTACl) (Fluka, ChiralSelect, $\geq 99.0 \%$ ) were used without further purification. The following were titrated according to the representative reference and by ${ }^{1} \mathrm{H}-\mathrm{NMR}$ titration according to Hoye et. al: ${ }^{3}$ tert-butyllithium $\left(t\right.$-BuLi) ${ }^{4} \quad 3$-chloroperbenzoic acid washed $(m$ CPBA) ${ }^{5}$ diisobutylaluminum hydride (DIBAL-H) ${ }^{6}$ Lithium triethylborohydride (super hydride), ${ }^{6,7}$ a solution of lithium aluminum hydride in tetrahydrofuran was prepared and titrated according Brown et al. ${ }^{7}$

${ }^{1} \mathrm{H}$ NMR, ${ }^{13} \mathrm{C}$ NMR, ${ }^{19} \mathrm{~F}$ NMR were recorded on Varian Unity $400\left(400 \mathrm{MHz},{ }^{1} \mathrm{H} ; 100\right.$ $\left.\mathrm{MHz},{ }^{13} \mathrm{C}\right)$, Varian Inova $500\left(500 \mathrm{MHz},{ }^{1} \mathrm{H}\right)$, and Varian VXR $500\left(499 \mathrm{MHz},{ }^{1} \mathrm{H} ; 125 \mathrm{MHz}\right.$ $\left.{ }^{13} \mathrm{C} ; 470 \mathrm{MHz},{ }^{19} \mathrm{~F}\right)$ spectrometer. Spectra were referenced to residual chloroform $\left(7.26 \mathrm{ppm},{ }^{1} \mathrm{H}\right.$; $\left.77.00 \mathrm{ppm},{ }^{13} \mathrm{C}\right)$, dimethyl sulfoxide $\left(2.50 \mathrm{ppm},{ }^{1} \mathrm{H} ; 39.51 \mathrm{ppm}{ }^{13} \mathrm{C}\right)$ and methanol (4.87 ppm, $\left.3.31 \mathrm{ppm}{ }^{1} \mathrm{H} ; 49.15 \mathrm{ppm}{ }^{13} \mathrm{C}\right)$. Chemical shifts are reported in ppm, multiplicities are indicated by s (singlet), d (doublet), t (triplet), q (quartet), sep (septet), m (multiplet), dd (doublet of doublets), ddd (doublet of doublet of doublets) dddd (doublet of doublet of doublet of doublets), tq (triplet of quartet), dt (doublet of triplet), td (triplet of doublets), nofoddd (non first order doublet of doublet of doublets), and br (broad). Coupling constants, $J$, are reported in Hertz. The University of Illinois Mass Spectrometer Center performed Mass spectroscopy. EI and CI mass spectra were performed on a 70-VSE spectrometer. ESI mass spectra were performed on a Micromass Quattro spectrometer. Data are reported in the form of $(\mathrm{m} / \mathrm{z})$. Infrared spectra (IR) were recorded on a Mattson Galaxy 5020 spectrophotometer in $\mathrm{NaCl}$ cells. Peaks are reported in $\mathrm{cm}^{-1}$ with indicated relative intensities: $\mathrm{s}$ (strong, 67-100\%); m (medium, 34-66\%); w (weak, 033\%). The University of Illinois Microanalytical Service Laboratory performed elemental analyses. Optical rotation data was obtained on a JASCO DIP-360 digital polarimeter and are reported as follows: concentration $(\mathrm{c}=\mathrm{g} / 100 \mathrm{~mL})$, and solvent. Analytical supercritical fluid chromatography (CSP-SFC) was performed on a Berger Instruments packed-column SFC with built-in photometric detector $(220 \mathrm{~nm})$ using Daicel Chiralpak OD, OJ, OB, AD and AS columns as well as a Regis Whelk-O1 column. Melting points (mp) were determined on a ThomasHoover capillary melting point apparatus in sealed tubes and are corrected. Ozonolyses were performed with a Welsbach Model T-816 Ozonator set at $55 \mathrm{~W} / 90 \mathrm{~V}$ with a $1 \mathrm{lpm}$ flow rate. Kugelrohr distillations were performed on a Büchi GKR-50 Kugelrohr and temperatures reported are air bath temperatures (ABT). 


\section{Literature Preparations}

Ethyl 4-(diethoxyphosphinyl) tiglate was prepared according to Kibayashi et. al. ${ }^{8}(S)-(-)-$ Citronellol was prepared following the procedure described by Noyori and co-workers. ${ }^{9}$ Allyltrichlorosilane was prepared following the procedure of Sakurai and co-workers. ${ }^{10} N, N^{\prime}-$ Dimethyl- $N, N$ '-bis-((3'a $R, 4$ 'a $R)$-7'-oxooctahydro-6'a,7'a-diaza-7'-phospha-cyclopenta[a]apentalene-7'-yl)-pentane-1,5-diamine $(R, R-26)$ was prepared according to Denmark and Fu. ${ }^{11}$ Di(tert-butyl)silyl ditriflate was prepared according to the method of Corey and Hopkins. ${ }^{12}$ Methyl (triphenylphosphoranylidene)acetate was prepared according to Hesse and Li. $^{13}$ Trimethylsilylethoxy chlorocarbonate (TEOC-Cl) was prepared following the procedure of Gioeli and co-workers. ${ }^{14}$ Tri- $O$-acetyl-D-glucal was prepared in accord with Roth and Pigman. ${ }^{15}$

\section{Scheme 1}

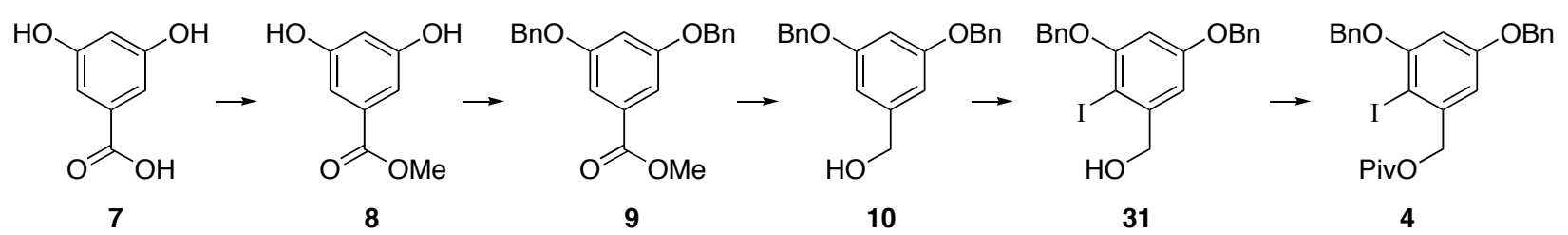

\section{Scheme 2}

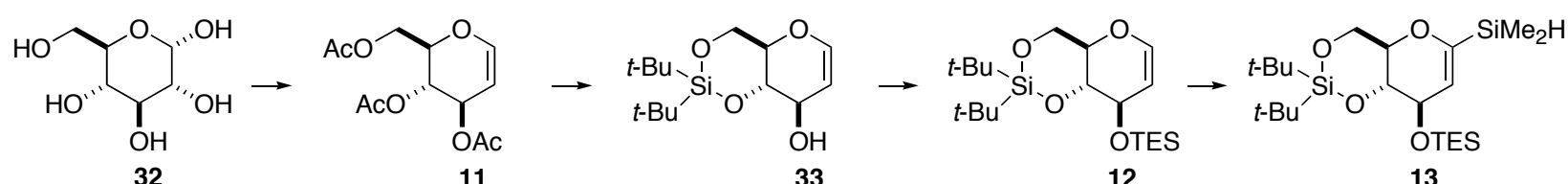

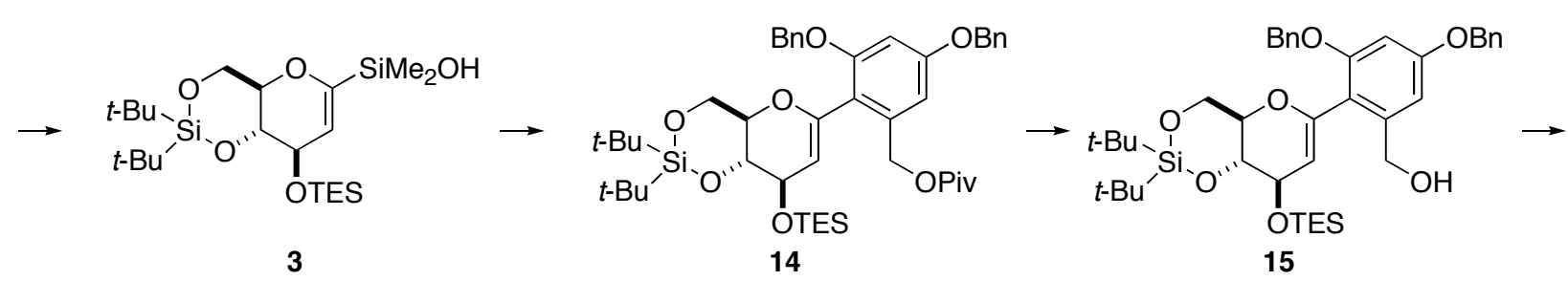




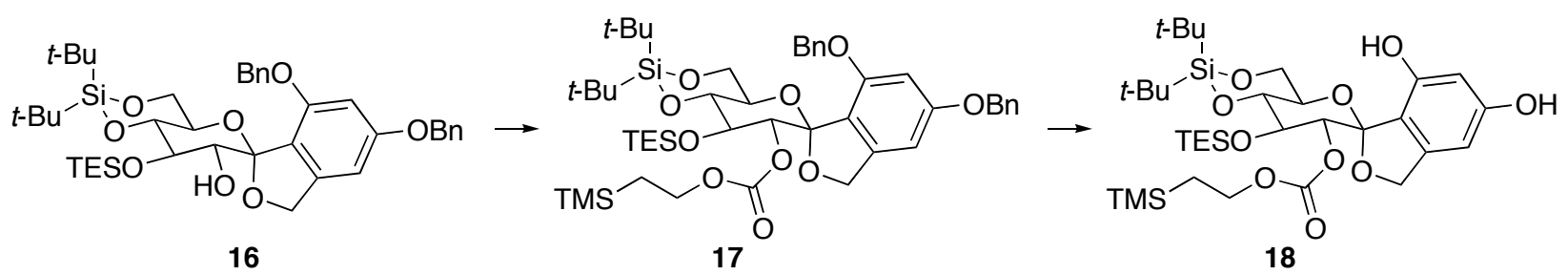

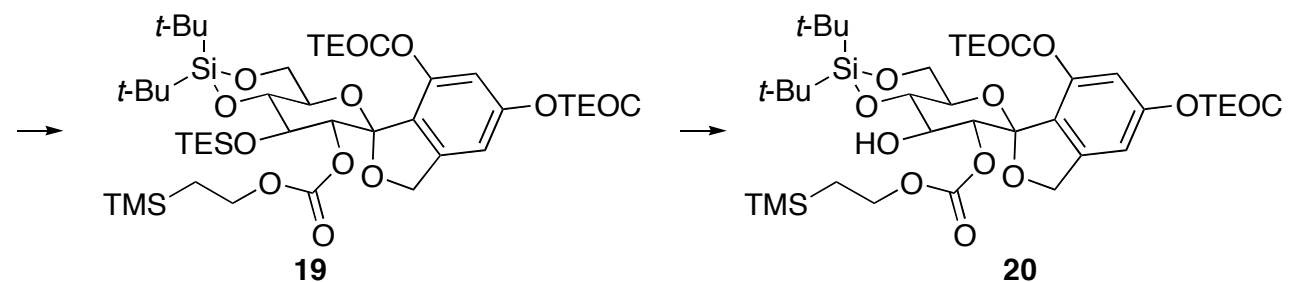

\section{Scheme 3}

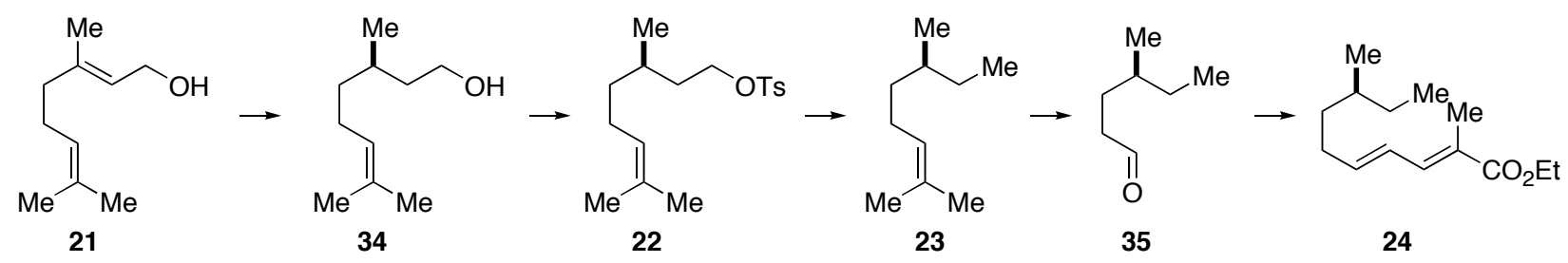

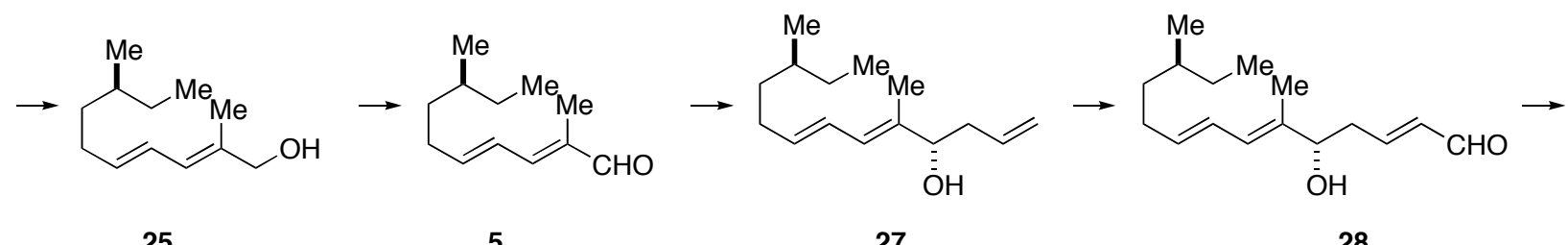

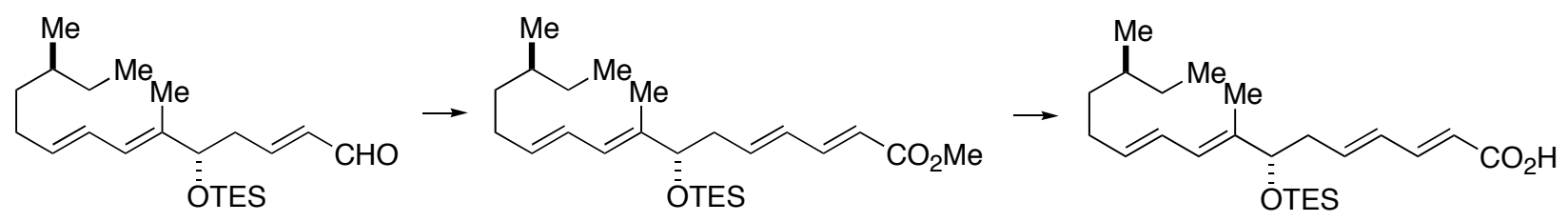

29

36

2

\section{Scheme 4}

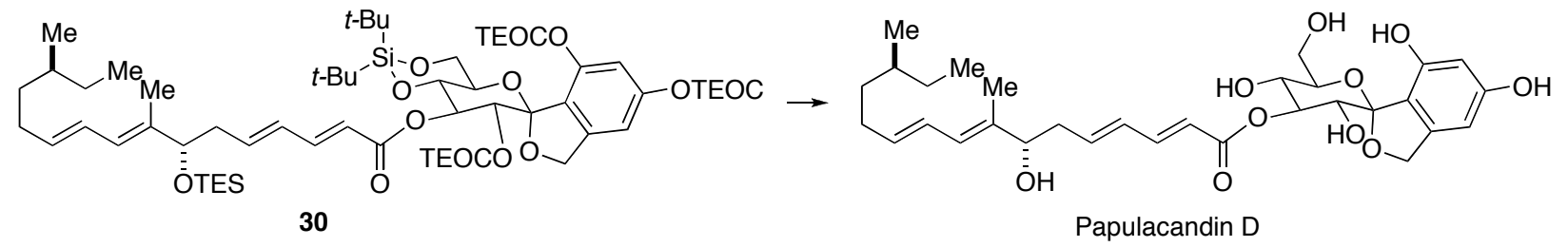




\section{Preparation of Aromatic Iodide 4}

\section{Preparation of Methyl 3,5-Dihydroxybenzoate (8) ${ }^{16}$ [CSR-IV-89]}<smiles>O=C(O)c1cc(O)cc(O)c1</smiles>

7
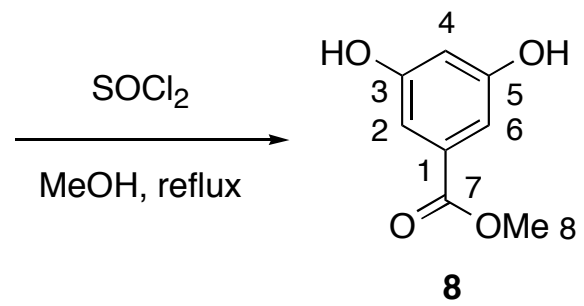

A 500-mL, three-necked, round-bottom flask, equipped with a Teflon-coated blade attached to an over-head stirrer, nitrogen inlet, reflux condenser bearing a drying tube, and a rubber septum which contains a needle vented to a mineral oil bubbler, was purged with nitrogen and charged with 3,5-dihydrobenzoic acid (7, $20 \mathrm{~g}, 130 \mathrm{mmol}, 1.0$ equiv), followed by the addition of freshly distilled $\mathrm{MeOH}(300 \mathrm{~mL})$. Thionyl chloride (11.4 mL, $157 \mathrm{mmol}, 1.2$ equiv) was added cautiously by syringe over $25 \mathrm{~min}$. to the rapidly stirring solution at room temperature. The nitrogen inlet was removed after the addition was complete and the nitrogen inlet was replaced with a rubber septum. The resulting solution was stirred at reflux (oil bath temperature $97^{\circ} \mathrm{C}$ ) for $2 \mathrm{~h}$. The contents were then transferred to a 500-mL, one-neck, roundbottom flask, and the $\mathrm{MeOH}$ was removed under reduced pressure by rotary evaporation. The resulting beige solid was dissolved in $\mathrm{MeOH}(50 \mathrm{~mL})$ and once again concentrated under reduced pressure by rotary evaporation, this procedure was repeated two more times and remaining volatiles were removed under high-vacuum $(0.03 \mathrm{mmHg})$ to afford $22.0 \mathrm{~g}(99 \%)$ of 8 as a powdery, beige solid.

Data for 8:

mp: $\quad 168-169^{\circ} \mathrm{C}(\mathrm{MeOH})$

1․ NMR: $\quad(400 \mathrm{MHz}, \mathrm{DMSO})$

$9.64(\mathrm{br}, 1 \mathrm{H},(\mathrm{OH})), 6.80(\mathrm{~d}, J=2.2 \mathrm{~Hz}, 2 \mathrm{H}, \mathrm{HC}(2)), 6.43$ (t, $J=2.2 \mathrm{~Hz}, 1 \mathrm{H}$, $\mathrm{HC}(4)), 3.77$ (s, 3H, $\left.\mathrm{H}_{3} \mathrm{C}(6)\right)$

${ }^{13} \mathrm{C}$ NMR: $\quad(126 \mathrm{MHz}, \mathrm{DMSO})$

166.3 (C(5)), 158.6 (C(3)), 131.3 (C(1)), 107.2 (C(4)), $107.1(\mathrm{C}(2)), 52.0(\mathrm{C}(6))$

IR: $\quad(\mathrm{KBr})$ 3560 (s), 3050 (s), 2910 (s), 1710 (s), 1590 (s), 1320 (m), 1280 (m)

MS: $\quad(\mathrm{EI}, 70 \mathrm{eV})$

$168\left(\mathrm{M}^{+}, 73\right), 137$ (100), 109 (39), 81 (17), 69 (27) 
TLC: $\quad R_{f} 0.43$ (hexanes/EtOAc, 1/1) [ $\left[\mathrm{SiO}_{2}, \mathrm{UV}, \mathrm{CAM}\right]$

Preparation of Methyl 3,5-Bis(phenylmethoxy)benzoate (9) ${ }^{16}$ [CSR-V-2]

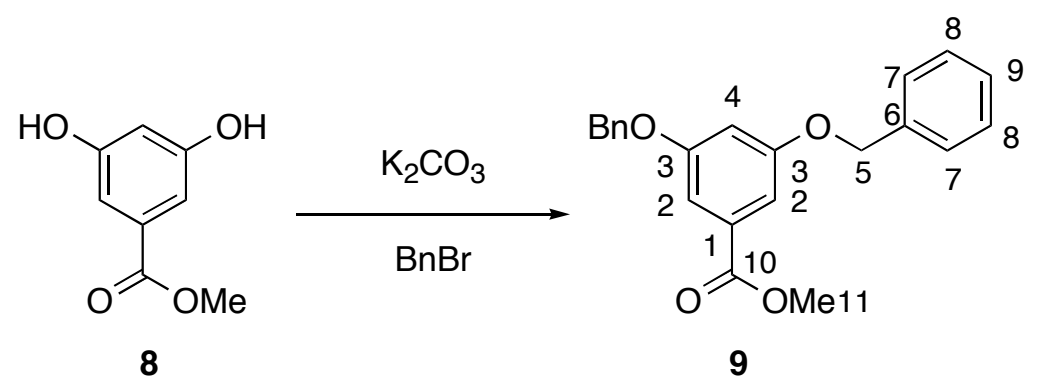

A 500-mL, single-necked, round-bottom flask, equipped with a large magnetic stir-bar and nitrogen inlet was purged with nitrogen and charged with 8 (15.0 g, $89.2 \mathrm{mmol}, 1.0$ equiv) and acetone $(100 \mathrm{~mL})$. Potassium carbonate $(37.0 \mathrm{~g}, 267.6 \mathrm{mmol}, 3.0$ equiv) was added and a brown mixture was observed. Then, benzyl bromide ( $32 \mathrm{~mL}, 267.6 \mathrm{mmol}, 3.0$ equiv) was added by syringe to the mixture at room temperature, the resulting mixture was stirred continuously at room temperature for $24 \mathrm{~h}$ (the mixture became a thick slurry over time, due to precipitation of $\mathrm{KBr}$, and for lager scale preparation an over-head stirrer was used). The contents were diluted with EtOAc $(150 \mathrm{~mL})$ and $\mathrm{H}_{2} \mathrm{O}(175 \mathrm{~mL})$. The aqueous layer was separated and extracted with EtOAc $(4 \times 150 \mathrm{~mL})$. The combined organic layers were washed with brine $(75 \mathrm{~mL})$, dried over anhydrous magnesium sulfate, filtered, and concentrated under reduced pressure to afford a brown solid which was recrystallized from tert-butyl methyl ether $(60 \mathrm{~mL})$ to provide $31 \mathrm{~g}$ (99\%) of 9 as a white crystalline powder.

Data for 9:

mp: $\quad 70-71{ }^{\circ} \mathrm{C}$ (TBME)

${ }^{1}$ H NMR: $\quad\left(500 \mathrm{MHz}, \mathrm{CDCl}_{3}\right)$

$7.45(\mathrm{~d}, J=7.1 \mathrm{~Hz}, 2 \mathrm{H}, \mathrm{HC}(7)), 7.41(\mathrm{t}, J=7.1 \mathrm{~Hz}, 2 \mathrm{H}, \mathrm{HC}(8)), 7.37$ (d, $J=7.1$ $\mathrm{Hz}, 2 \mathrm{H}, \mathrm{HC}(9)), 7.34$ (d, $J=2.2 \mathrm{~Hz}, 2 \mathrm{H}, \mathrm{HC}(2)), 6.834$ (t, $J=2.4 \mathrm{~Hz}, 1 \mathrm{H}, \mathrm{HC}(4))$, 5.09 (s, 4H, HC(5)), 3.92 (s, 3H, $\mathrm{HC}(11)$ )

${ }^{13} \mathrm{C} \mathrm{NMR}: \quad\left(126 \mathrm{MHz}, \mathrm{CDCl}_{3}\right)$

$166.2(\mathrm{C}(10)), 159.7(\mathrm{C}(3)), 136.4(\mathrm{C}(6)), 132.0(\mathrm{C}(1)), 128.6(\mathrm{C}(8)), 128.1$

$(\mathrm{C}(9)), 127.5(\mathrm{C}(7)), 108.3(\mathrm{C}(2)), 107.2(\mathrm{C}(4)), 70.2(\mathrm{C}(5)), 52.2(\mathrm{C}(11))$

IR: $\quad(\mathrm{KBr})$

1714 (s), 1724 (s), 1597 (s), 1439 (s), 1378 (s), 1252 (s), 1083 (s), 966 (w), 868 
(w), $763(\mathrm{~s})$

MS: $\quad(\mathrm{EI}, 70 \mathrm{eV})$

$$
348\left(\mathrm{M}^{+}, 13\right), 100 \text { (100), } 62 \text { (3) }
$$

TLC: $\quad R_{f} 0.39$ (hexanes/EtOAc, 3/1) $\left[\mathrm{SiO}_{2}, \mathrm{UV}, \mathrm{CAM}\right]$

\section{Preparation of 3,5-Bis(phenylmethoxy)benzenemethanol (10) ${ }^{16}$ [CSR-IV-95]}
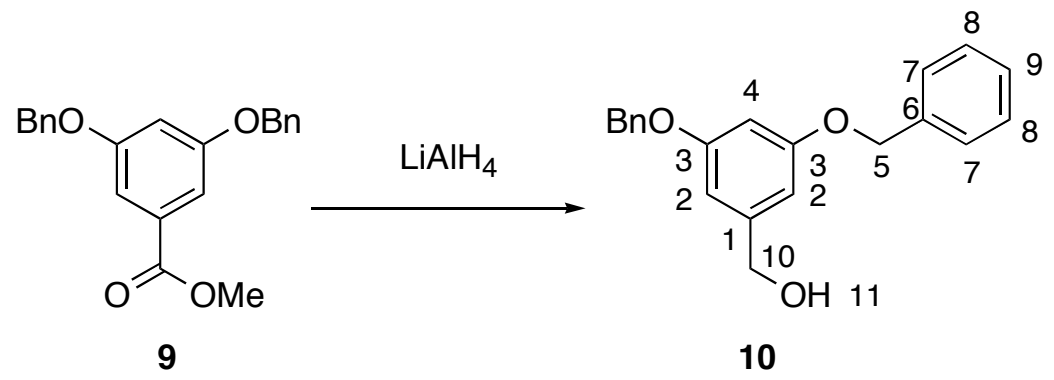

A 250-mL, two-necked, round-bottom flask, equipped with a magnetic stir-bar, argon inlet and a rubber septum was purged with argon and charged with 9 (10.0 g, $28.7 \mathrm{mmol}, 1.0$ equiv) followed by THF $(20 \mathrm{~mL})$. The solution was then cooled to $0{ }^{\circ} \mathrm{C}$ using an ice bath. Lithium aluminum hydride in THF $(0.9 \mathrm{M}, 70 \mathrm{~mL}, 63.1 \mathrm{mmol}, 2.2 \text { equiv })^{7}$ was added cautiously by syringe over $30 \mathrm{~min}$ (maintaining the internal temperature between 10 to $15^{\circ} \mathrm{C}$ ). After the addition was complete the resulting solution was stirred at room temperature for $3 \mathrm{~h}$, whereupon the solution was once again cooled to $0{ }^{\circ} \mathrm{C}$ and $\mathrm{H}_{2} \mathrm{O}(2.5 \mathrm{~mL}), 15 \% \mathrm{NaOH}(2.5 \mathrm{~mL}), \mathrm{H}_{2} \mathrm{O}(7.5$ $\mathrm{mL}$ ) were added sequentially. Upon warming to room temperature, the white suspension was filtered through Celite $(5 \mathrm{~g})$ and the Celite pad was washed with THF ( $40 \mathrm{~mL})$. The filtrate was concentrated under reduced pressure by rotary evaporation. The resulting white suspension was dissolved in toluene $(20 \mathrm{~mL})$ and once again concentrated under reduced pressure by rotary evaporation, this procedure was repeated two more times and remaining volatiles were removed under high vacuum $(0.06 \mathrm{mmHg})$ to afford a white solid. The solid was recrystallized from hot absolute ethanol $(60 \mathrm{~mL})$ to afford $9.0 \mathrm{~g}(98 \%)$ of $\mathbf{1 0}$ as white needles.

Data for 10:

mp: $\quad 79-80^{\circ} \mathrm{C}(\mathrm{EtOH})$

${ }^{1}$ H NMR: $\quad\left(500 \mathrm{MHz}, \mathrm{CDCl}_{3}\right)$

7.34 (m, 10H, $\mathrm{HC}(7,8,9)), 6.63$ (d, $J=2.2 \mathrm{~Hz}, 2 \mathrm{H}, \mathrm{H} \mathrm{C}(2)), 6.56$ (t, $J=2.3 \mathrm{~Hz}$, 1H, HC(4)), 5.04 (s, $4 \mathrm{H}, \mathrm{HC}(5)), 4.62$ (d, $J=5.2 \mathrm{~Hz}, 2 \mathrm{H}, \mathrm{HC}(10)), 1.90$ (t, $J=$ $5.2 \mathrm{~Hz}, 1 \mathrm{H},(\mathrm{OH}))$ 
${ }^{13}$ C NMR: $\quad\left(126 \mathrm{MHz}, \mathrm{CDCl}_{3}\right)$

$160.1(\mathrm{C}(3)), 143.4(\mathrm{C}(6)), 136.7$ (C(1)), $128.6(\mathrm{C}(8)), 127.9$ (C(9)), $127.5(\mathrm{C}(7))$, $105.6(\mathrm{C}(2)), 101.2(\mathrm{C}(4)), 69.9(\mathrm{C}(5)), 65.2(\mathrm{C}(10))$

IR: $\quad(\mathrm{KBr})$

3303 (br), 3325 (m), 2923 (s), 1593 (m), 1497 (m), 1352 (m), 1285 (m), 1159 (s), $833(\mathrm{~m})$

MS: $\quad(\mathrm{EI}, 70 \mathrm{eV})$

$320\left(\mathrm{M}^{+}, 17\right), 181(11), 91(100)$

TLC: $\quad R_{f} 0.30$ (hexanes/EtOAc, 3/1) [ $\mathrm{SiO}_{2}, \mathrm{UV}, p$-anisaldehyde]

\section{Preparation of 2-Iodo-3,5-bis(phenylmethoxy)benzenemethanol (31) [CSR-V-1]}

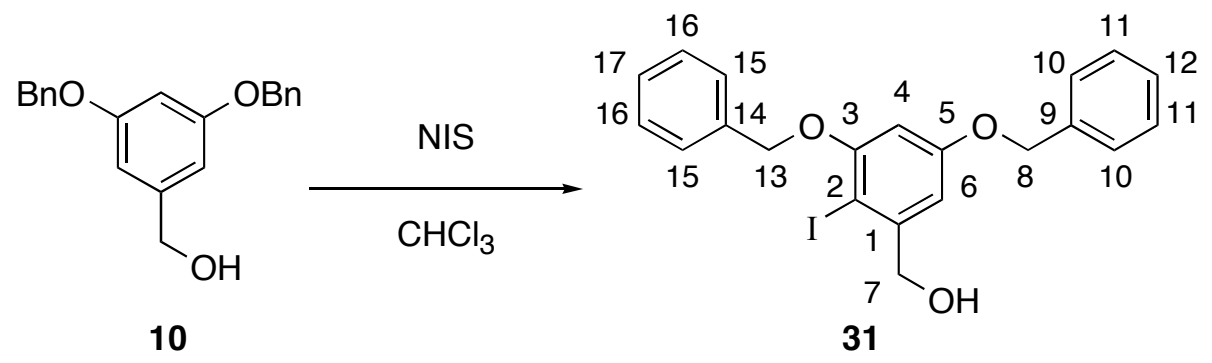

A 250-mL, single-necked, round-bottom flask, equipped with a magnetic stir-bar, argon inlet, was purged with argon and charged with 10 (5.0 g, $15.6 \mathrm{mmol}, 1.0$ equiv) followed by freshly distilled chloroform $(34 \mathrm{~mL})$. To this solution, $N$-iodosuccinimide $(4.21 \mathrm{~g}, 18.7 \mathrm{mmol}$, 1.2 equiv) was added. The flask was wrapped in aluminum foil and stirred at room temperature for $18 \mathrm{~h}$. The mixture was diluted with EtOAc $(50 \mathrm{~mL})$ and the pink suspension was eluted through Celite $(5 \mathrm{~g})$ washing the Celite pad with EtOAc $(100 \mathrm{~mL})$. Then $\mathrm{H}_{2} \mathrm{O}(20 \mathrm{~mL})$ was added and the aqueous layer was separated and extracted with EtOAc ( 2 x $15 \mathrm{~mL})$. The combined organic layers were washed with saturated, aqueous sodium thiosulfate $(30 \mathrm{~mL})$ and brine $(40 \mathrm{~mL})$, dried over anhydrous magnesium sulfate, filtered, and concentrated under reduced pressure by rotary evaporation. The white residue was recrystallized from isopropyl acetate $(20 \mathrm{~mL})$ adding hot heptane $(80 \mathrm{~mL})$, until the solution became turbid and then cooled in an ice bath for $2 \mathrm{~h}$, to provide $6.3 \mathrm{~g}(91 \%)$ of $\mathbf{3 1}$, as a white crystalline powder.

\section{Data for 31:}

mp: $\quad 113-114^{\circ} \mathrm{C}(4 / 1$ heptane/isopropyl acetate) 
${ }^{1} \underline{\mathrm{H} N M R}: \quad\left(500 \mathrm{MHz}, \mathrm{CDCl}_{3}\right)$

7.49 (d, $J=7.5 \mathrm{~Hz}, 2 \mathrm{H}, \mathrm{HC}(16,11)), 7.41-7.31$ (m, 8H, $\mathrm{HC}(15,17,12,10)$ ),

$6.84(\mathrm{~d}, J=2.6 \mathrm{~Hz}, 1 \mathrm{H}, \mathrm{HC}(6)), 6.50$ (d, $J=2.6 \mathrm{~Hz}, 1 \mathrm{H}, \mathrm{HC}(4)), 5.10$ (s, $2 \mathrm{H}$,

HC(13)), 5.05 (s, 2H, HC(8)), 4.69 (d, $J=6.2 \mathrm{~Hz}, 2 \mathrm{H}, \mathrm{HC}(7)$ ), 2.12 (t $J=6.2$

$\mathrm{Hz}, 1 \mathrm{H},(\mathrm{OH}))$

${ }^{13}$ C NMR: $\quad\left(126 \mathrm{MHz}, \mathrm{CDCl}_{3}\right)$

160.4 (C(3)), 157.7 (C(5)), 144.8 (C(1)), 136.5 (C(14)), 136.3 (C(9)), 128.7

(C(16), $128.6(\mathrm{C}(11)), 128.2(\mathrm{C}(15), 127.9(\mathrm{C}(17)), 127.6(\mathrm{C}(10)), 126.9(\mathrm{C}(12))$,

$106.6(\mathrm{C}(6)), 100.3(\mathrm{C}(4)), 78.8(\mathrm{C}(2)), 70.9(\mathrm{C}(13)), 70.3(\mathrm{C}(8)), 69.6(\mathrm{C}(7))$

IR: $\quad(\mathrm{KBr})$

3305 (br), 1580 (s), 1498 (m), 1424 (m), 1279 (m), 1169 (s), 1055 (s), $1010(\mathrm{~s})$,

$732(\mathrm{~m})$

MS: $\quad(E I, 70 \mathrm{eV})$

$446\left(\mathrm{M}^{+}, 12\right), 181(10), 91(100), 65(10)$

TLC: $\quad R_{f} 0.24$ (hexanes/EtOAc, $10 / 1$ ) $\left[\mathrm{SiO}_{2}, \mathrm{UV}\right]$

Analysis: $\quad \mathrm{C}_{21} \mathrm{H}_{19} \mathrm{IO}_{3}(446.23)$

Calcd: $\quad$ C, $56.52 \% ; \quad H, 4.29 \% ; \quad$ I, $28.44 \%$

Found: $\quad$ C, $56.43 \% ; \quad H, 4.24 \% ; \quad$ I, $28.09 \%$

\section{Preparation of 2-Iodo-3,5-bis(phenylmethoxy-2',2'-dimethylpropanoate)benzenemethanol} (4) [CSR-V-27]

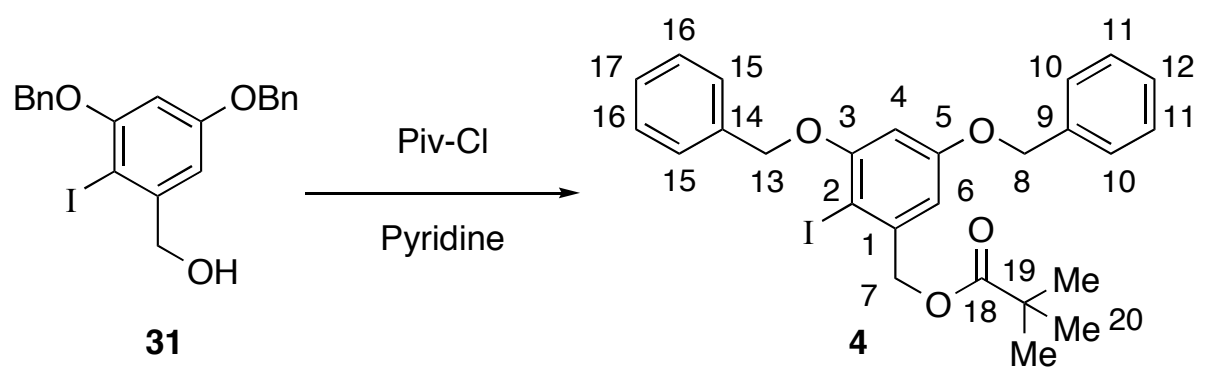

A 100-mL, two-necked, round-bottom flask, equipped with a magnetic stir-bar, argon inlet, and a rubber septum, was purged with argon and charged with 31 (4.0 g, 8.9 mmol, 1.0 equiv) followed by $\mathrm{CH}_{2} \mathrm{Cl}_{2}(35 \mathrm{~mL})$. To this solution, pyridine (1.08 $\mathrm{mL}, 13.4 \mathrm{mmol}, 1.5$ equiv) and pivaloyl chloride (1.3 mL, $10.6 \mathrm{mmol}, 1.2$ equiv) were added sequentially. The light-yellow solution was stirred at room temperature for $3 \mathrm{~h}$. The solution was diluted with $\mathrm{H}_{2} \mathrm{O}(60 \mathrm{~mL})$ and 
the aqueous layer was separated and extracted with $\mathrm{CH}_{2} \mathrm{Cl}_{2}(3 \times 40 \mathrm{~mL})$. The combined organic layers were washed with saturated, aqueous sodium bicarbonate $(30 \mathrm{~mL})$ and brine $(40 \mathrm{~mL})$, dried over anhydrous magnesium sulfate, filtered, and concentrated under reduced pressure by rotary evaporation. The oily residue was purified by column chromatography $\left(\mathrm{SiO}_{2}(40 \times 180\right.$ $\mathrm{mm})$, hexanes/EtOAc, 3/1). Further purification by recrystallization from hexane (15 $\mathrm{mL})$ afforded $4.5 \mathrm{~g}(94 \%)$ of $\mathbf{4}$ as white crystalline rhombuses.

Data for 4:

$\underline{\mathrm{mp}}: \quad 50-51{ }^{\circ} \mathrm{C}$ (hexane)

${ }^{1} \underline{\mathrm{H} \mathrm{NMR}}: \quad\left(500 \mathrm{MHz}, \mathrm{CDCl}_{3}\right)$

$7.49(\mathrm{~d}, J=7.6 \mathrm{~Hz}, 2 \mathrm{H}, \mathrm{HC}(16,11)), 7.41-7.28$ (m, 8H, HC(15, 17, 12, 10)), $6.69(\mathrm{~d}, J=2.7 \mathrm{~Hz}, 1 \mathrm{H}, \mathrm{HC}(6)), 6.52$ (d, $J=2.4 \mathrm{~Hz}, 1 \mathrm{H}, \mathrm{HC}(4)), 5.13$ (s, 2H, HC(13)), 5.12 (s, 2 , HC(8)), 5.04 (s, 2 H, HC (7)), 1.25 (s, 9H, HC(20))

${ }^{13} \mathrm{C} \mathrm{NMR}: \quad\left(126 \mathrm{MHz}, \mathrm{CDCl}_{3}\right)$

177.9 (C(18)), 160.1 (C(3)), 157.9 (C(5)), 140.8 (C(1)), 136.4 (C(14)), 136.3

(C(9), 128.7 (C(16)), 128.6 (C(11), 128.2 (C(15)), 127.9 (C(17)), 127.5 (C(10)), 127.0 (C(12)), 107.2 (C(6)), 100.5 (C(4)), 79.8 (C(11)), 71.0 (C( 13)), 70.3 (C(8)), 70.2 (C(7)), 38.9 (C(19)), 27.3 (C(20))

IR: $\quad(\mathrm{KBr})$

3075 (w), 2927 (w), 1720 (s), 1584 (2), 1496 (m), 1465 (m), 1281 (s), 1158 (s), $732(\mathrm{~s})$

MS: $\quad(\mathrm{EI}, 70 \mathrm{eV})$ $530\left(\mathrm{M}^{+}, 6\right), 446$ (9), 404 (2), 348 (100), 181 (11), 91 (100)

TLC: $\quad R_{f} 0.50$ (hexanes/EtOAc, 10/1) $\left[\mathrm{SiO}_{2}, \mathrm{KMnO}_{4}\right]$

Analysis: $\quad \mathrm{C}_{26} \mathrm{H}_{27} \mathrm{IO}_{4}(530.39)$

Calcd: $\quad$ C, $58.88 \% ; \quad$ H, $5.13 \%$; $\quad$ I, $23.93 \%$

Found: C, $58.96 \%$; H, $5.15 \%$; $\quad$ I, $23.79 \%$ 


\section{Preparation of Spirocyclic $C$-Arylglycoside 20}

Preparation of 1,5-Anhydro-4,6-O-[bis(1,1-dimethylethyl)silylene]-2-deoxy- $D$-arabino-1hexenitol (33) [CSR-V-95] ${ }^{18}$

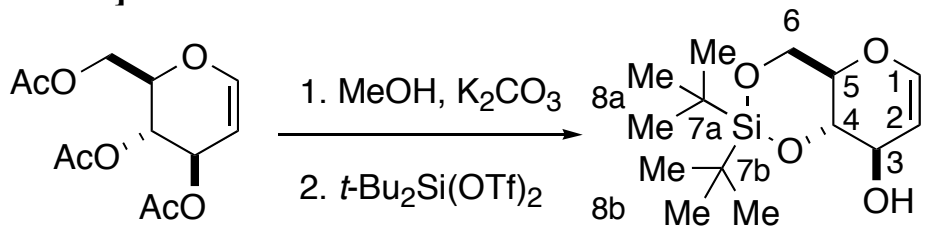

11

33

To a 500-mL, two-necked, round-bottom flask, fitted with a nitrogen inlet, a septum, and magnetic stir-bar was added triacetoxyglucal 11 (10.0 g, $36.6 \mathrm{mmol}, 1.0$ equiv) followed by dry $\mathrm{MeOH}(37 \mathrm{~mL})$. Then $\mathrm{K}_{2} \mathrm{CO}_{3}(0.051 \mathrm{~g}, 0.369 \mathrm{mmol}, 0.01$ equiv) was added. The light-yellow solution was stirred at room temperature for $1 \mathrm{~h}$. The volatiles were removed under reduced pressures using rotary evaporation. The resulting yellow viscous reside was dissolved in $\mathrm{CHCl}_{3}$ $(100 \mathrm{~mL})$ and once again concentrated under reduced pressure by rotary evaporation. This procedure was repeated two more times and the remaining volatiles were removed under high vacuum $(0.06 \mathrm{mmHg})$ for approximately $2 \mathrm{~h}$. Then dry dimethylformamide $(37 \mathrm{~mL})$ was added to the crude yellow syrup, followed by 2,6-lutidine $(12.9 \mathrm{~mL}, 110.8 \mathrm{mmol}, 3.0$ equiv) was added and the solution was cooled to $-15^{\circ} \mathrm{C}$ using an acetone/ice bath. Di(tert-butyl)silyl ditriflate ${ }^{12}$ (14.8 mL, $40.6 \mathrm{mmol}, 1.1$ equiv) was added. The contents were warmed to room temperature and stirred for $1.5 \mathrm{~h}$. This solution was diluted with $\mathrm{H}_{2} \mathrm{O}(200 \mathrm{~mL})$ and extracted with $\mathrm{Et}_{2} \mathrm{O}(3 \mathrm{x}$ $100 \mathrm{~mL})$. The combined organic extracts were washed with brine $(100 \mathrm{~mL})$, dried over $\mathrm{Na}_{2} \mathrm{SO}_{4}$ and filtered. The solvent was removed by rotary evaporation to afford a light-yellow oil. The crude reside was purified by column chromatography $\left(\mathrm{SiO}_{2}(60\right.$ x $220 \mathrm{~mm})$, hexanes/EtOAc, 15/1)), which afforded $9.4 \mathrm{~g}(89 \%)$ of 33 as a white powder.

Data for 33:

mp: $\quad 67-69^{\circ} \mathrm{C}($ hexanes/EtOAc, $15 / 1)$

${ }^{1} \mathrm{H} \mathrm{NMR}: \quad\left(500 \mathrm{MHz}, \mathrm{CDCl}_{3}\right)$

$6.27(\mathrm{dd}, J=6.5,2.0 \mathrm{~Hz}, 1 \mathrm{H}, \mathrm{CH}(1)) ; 4.76(\mathrm{dd}, J=6.5,2.0 \mathrm{~Hz}, 1 \mathrm{H}, \mathrm{CH}(2)) ; 4.30$ (d, $J=6.5 \mathrm{~Hz}, 1 \mathrm{H}, \mathrm{CH}(3))$; 4.18 (dd, $J=10.7,5.4 \mathrm{~Hz}, 1 \mathrm{H}, \mathrm{CHe}(6)) ; 3.96$ (t, $J=$ $10.7 \mathrm{~Hz}, 1 \mathrm{H}, \mathrm{CHa}(6)) ; 3.92(\mathrm{dd}, J=10.7,6.5 \mathrm{~Hz}, 1 \mathrm{H}, \mathrm{CH}(4)) ; 3.84$ (dt, $J=10.7$, $5.4 \mathrm{~Hz}, 1 \mathrm{H}, \mathrm{CH}(5)) ; 2.41(\mathrm{~s}, 1 \mathrm{H},(\mathrm{OH})) ; 1.08$ (s, 9H, $\mathrm{C}\left(\mathrm{CH}_{3}\right)_{3}(8 \mathrm{a}$ or $\left.8 \mathrm{~b})\right) ; 1.00(\mathrm{~s}$, $9 \mathrm{H}, \mathrm{C}\left(\mathrm{CH}_{3}\right)_{3}(8 \mathrm{a}$ or $\left.8 \mathrm{~b})\right)$ 
${ }^{13} \mathrm{C} \mathrm{NMR}: \quad\left(126 \mathrm{MHz}, \mathrm{CDCl}_{3}\right)$

$143.9(\mathrm{C}(1)) ; 103.2(\mathrm{C}(2)) ; 77.6(\mathrm{C}(4)) ; 72.5(\mathrm{C}(5)) ; 70.4(\mathrm{C}(3)) ; 65.9(\mathrm{C}(6)) ; 27.6$

$(\mathrm{C}(8 \mathrm{a}$ or $8 \mathrm{~b})) ; 27.1(\mathrm{C}(8 \mathrm{a}$ or $8 \mathrm{~b})) ; 22.9(\mathrm{C}(7 \mathrm{a}$ or $7 \mathrm{~b})) ; 20.0(\mathrm{C}(7 \mathrm{a}$ or $7 \mathrm{~b}))$

IR: $\quad$ (film)

3430 (m); 2963 (s); 2891(s); 1647 (m); 1473 (m); 1388 (w); 1364 (w); 1233 (m);

1159 (m); 1121 (s); 1098 (s); 1031 (w); 1012 (w); 994 (w); 871 (s); 826 (s); 767

(s); $653(\mathrm{~s})$

MS: $\quad(\mathrm{EI}, 70 \mathrm{eV})$

286 (M (13)); 230 (17); 229 (100); 199 (12); 157 (41); 119 (15); 115 (14); 103

(11); 91(11); 81(26); 77 (32); 57 (12)

Opt. Rot.: $\quad[\alpha]_{\mathrm{D}}^{24}-12.31(\mathrm{c}=0.79, \mathrm{EtOH})$

TLC: $\quad R_{f} 0.16$ (hexanes/EtOAc, 20/1) [ $\left.\mathrm{SiO}_{2}, \mathrm{UV}, \mathrm{KMnO}_{4}\right]$

HRMS: $\quad \mathrm{C}_{14} \mathrm{H}_{26} \mathrm{O}_{4} \mathrm{Si}(286.44)$

Calcd: $\quad 286.1600$

Found: $\quad 286.1599$

\section{Preparation of 1,5-Anhydro-4,6-O-[bis(1,1-dimethylethyl)silylene]-2-deoxy-3-O- (triethylsilyl)-D-arabino-1-hexenitol (12) [CSR-V-96]}

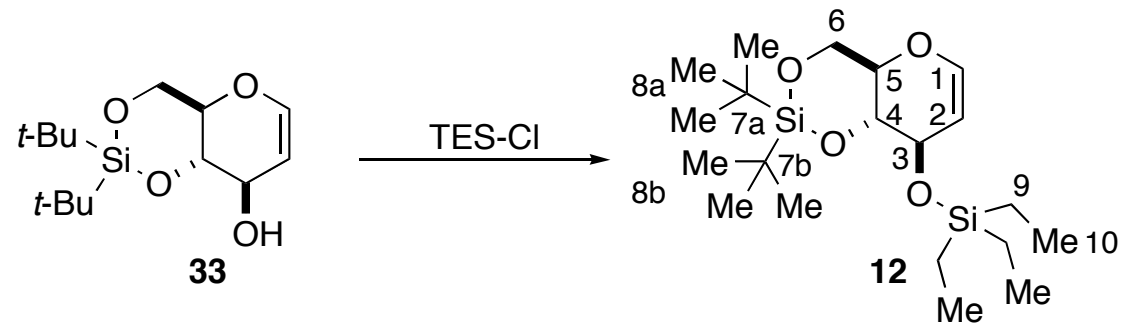

To a $500-\mathrm{mL}$, two-necked, round-bottom flask fitted with a nitrogen inlet, septum, and magnetic stir-bar was added alcohol 33 ( $8.9 \mathrm{~g}, 31.1 \mathrm{mmol}$, 1.0 equiv) followed by $\mathrm{CH}_{2} \mathrm{Cl}_{2}$ (100 $\mathrm{mL}$ ). Pyridine ( $3.77 \mathrm{~mL}, 46.7 \mathrm{mmol}, 1.2$ equiv) was added along with triethylchlorosilane (6.27 $\mathrm{mL}, 37.4 \mathrm{mmol}, 1.2$ equiv) and the colorless solution was stirred at room temperature for $4 \mathrm{~h}$. To this solution was added saturated, aqueous $\mathrm{NaHCO}_{3}(300 \mathrm{~mL})$. The aqueous layer was extracted with $\mathrm{CH}_{2} \mathrm{Cl}_{2}(3 \times 50 \mathrm{~mL})$. The combined organic layers were washed with brine $(100 \mathrm{~mL})$, dried over $\mathrm{Na}_{2} \mathrm{SO}_{4}$, filtered and concentrated under reduced pressure using rotary evaporation. The 
crude yellow oil was purified by column chromatography $\left(\mathrm{SiO}_{2}(60\right.$ x $220 \mathrm{~mm})$, hexanes/EtOAc, 15/1)) to afford $11.5 \mathrm{~g}(92 \%)$ of $\mathbf{1 2}$ as a colorless oil.

\section{Data for 12:}

bp: $\quad 145^{\circ} \mathrm{C}\left(6.5 \times 10^{-5} \mathrm{mmHg}, \mathrm{ABT}\right)$

${ }^{1}$ H NMR: $\quad\left(500 \mathrm{MHz}, \mathrm{CDCl}_{3}\right)$

$6.23(\mathrm{dd}, J=6.0,2.0 \mathrm{~Hz}, 1 \mathrm{H}, \mathrm{CH}(1)) ; 4.61(\mathrm{dd}, J=6.0,2.0 \mathrm{~Hz}, 1 \mathrm{H}, \mathrm{CH}(2)) ; 4.28$ $(\mathrm{dd}, J=7.0,2.0 \mathrm{~Hz}, 1 \mathrm{H}, \mathrm{CH}(3)) ; 4.15$ (dd, $J=10.5,5.0 \mathrm{~Hz}, 1 \mathrm{H}, \mathrm{CH}(6 \mathrm{e})) ; 3.96$ $(\mathrm{dd}, J=10.4,7.0 \mathrm{~Hz}, 1 \mathrm{H}, \mathrm{CH}(4)) ; 3.95$ (t, $J=10.5 \mathrm{~Hz} 1 \mathrm{H}, \mathrm{CH}(6 \mathrm{a})) ; 3.80$ (dt, $J=$ 10.4, $5.0 \mathrm{~Hz}, 1 \mathrm{H}, \mathrm{CH}(5)) ; 1.07\left(\mathrm{~s}, 9 \mathrm{H}, \mathrm{C}\left(\mathrm{CH}_{3}\right)_{3}(8 \mathrm{a}\right.$ or $\left.8 \mathrm{~b})\right) ; 1.00(\mathrm{~s}, 9 \mathrm{H}$, $\mathrm{C}\left(\mathrm{CH}_{3}\right)_{3}(8 \mathrm{a}$ or $\left.8 \mathrm{~b})\right) ; 0.99\left(\mathrm{t}, J=8.0 \mathrm{~Hz}, 9 \mathrm{H}, \mathrm{CH}_{3}(10)\right) ; 0.66(\mathrm{dq}, J=15.0,8.0 \mathrm{~Hz}$, $\left.6 \mathrm{H}, \mathrm{CH}_{2}(9)\right)$

${ }^{13} \mathrm{C} \mathrm{NMR}: \quad\left(126 \mathrm{MHz}, \mathrm{CDCl}_{3}\right)$

142.97 (C(1)); 105.13 (C(2)); 77.13 (C(4)); 72.77 (C(5)); 70.51 (C(3)); 65.84 $(\mathrm{C}(6)) ; 27.36(\mathrm{C}(8 \mathrm{a}$ or $8 \mathrm{~b})) ; 26.08(\mathrm{C}(8 \mathrm{a}$ or $8 \mathrm{~b})) ; 22.67(\mathrm{C}(7 \mathrm{a}$ or $7 \mathrm{~b})) ; 19.74(\mathrm{C}(7 \mathrm{a}$ or $7 \mathrm{~b})) ; 6.75(\mathrm{C}(9)) ; 4.76(\mathrm{C}(10))$

IR: (film)

2957 (s); 2935 (s); 2860 (s); 2878 (s); 1649 (m); 1473 (m); 1414 (w); 1391 (m);

1364 (w); 1234 (s); 1162 (s); 1123 (s); 1109 (s); 1002 (s); 969 (w); 880 (s); 826

(s); $735(\mathrm{~s}) ; 653(\mathrm{~s})$

MS: $\quad(\mathrm{CI}, 130 \mathrm{eV})$

$401\left(\left(\mathrm{M}^{+}+1\right)(10)\right) ; 371$ (14); 341 (18); 289 (13); 270 (18); 269 (76); 267 (15);

217 (22); 201 (15); 187 (30); 103 (10); 81 (100)

Opt. Rot.: $\quad[\alpha]_{\mathrm{D}}^{24}-46.80\left(\mathrm{c}=1.23, \mathrm{CHCl}_{3}\right)$

TLC: $\quad R_{f} 0.60$ (hexanes/EtOAc, $\left.10 / 1\right)\left[\mathrm{SiO}_{2}, \mathrm{CAM}\right]$

Analysis: $\quad \mathrm{C}_{20} \mathrm{H}_{40} \mathrm{O}_{4} \mathrm{Si}_{2}(400.70)$

Calcd: $\quad$ C, $59.87 \%$; $\quad$ H, $10.35 \%$

Found: $\quad$ C, $59.95 \%$; $\quad H, 10.06 \%$ 


\section{Preparation of 1,5-Anhydro-4,6- $O$-[bis(1,1-dimethylethyl)silylene]-2-deoxy-1- $C$ - (dimethyl(hydrido))-3-O-(triethylsilyl)-D-arabino-1-hexenitol (13) [CSR-V-66]}

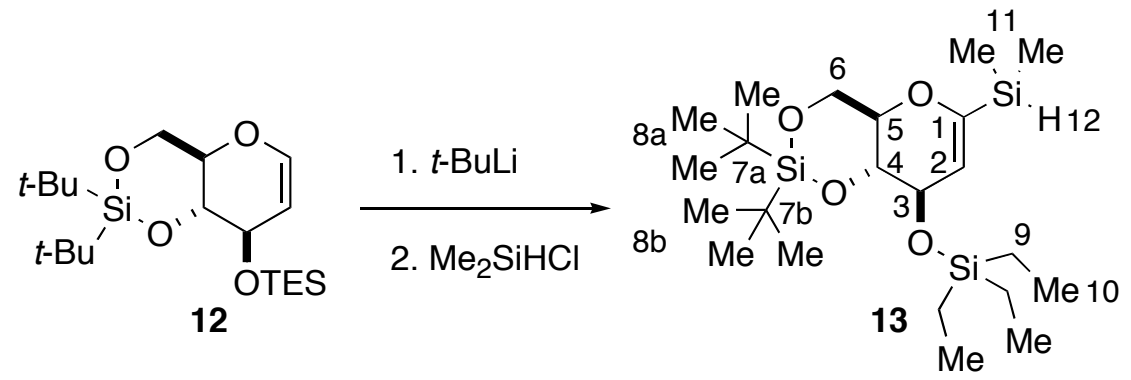

To a 25-mL, one-necked, round-bottom flask fitted with an argon inlet adaptor with septum, and magnetic stir-bar was charged with 12 (1.90 g, $4.7 \mathrm{mmol}, 1.0$ equiv), followed by THF (3.8 mL). The contents were cooled to $-78{ }^{\circ} \mathrm{C}$ using a 2-propanol/dry-ice bath. Then tertbutyllithium (1.51 $\mathrm{M}$ in pentane, $3.77 \mathrm{~mL}, 5.69 \mathrm{mmol}, 1.2$ equiv) was added drop wise. The solution turned bright yellow and became heterogeneous after the addition. The resulting solution was warmed to $-50{ }^{\circ} \mathrm{C}$ and was stirred for $30 \mathrm{~min}$., until the contents became homogenous. To a separate 50-mL, round-bottom flask, equipped with a magnetic stir-bar and an argon inlet with septum, dimethylchlorosilane $(672 \mu \mathrm{L}, 6.16 \mathrm{mmol}, 1.3$ equiv) was added and the contents were cooled to $-72{ }^{\circ} \mathrm{C}$. The solution of metalated 12 added into the solution of dimethylchlorosilane via cannula. The white mixture was warmed to room temperature with stirring for $1 \mathrm{~h}$. The suspension was then cooled to $0{ }^{\circ} \mathrm{C}$ (ice-bath) and quenched with saturated sodium bicarbonate $(20 \mathrm{~mL})$. The aqueous layer was extracted with EtOAc $(3 \mathrm{x} 10 \mathrm{~mL})$. The combined organic extracts were washed with brine $(15 \mathrm{~mL})$, dried and concentrated to a colorless oil. The crude contents were purified by column chromatography $\left(\mathrm{SiO}_{2}(40 \times 180\right.$ $\mathrm{mm}$ ), hexanes/EtOAc, 15/1), which afforded $2.1 \mathrm{~g}(94 \%)$ of $\mathbf{1 3}$ as a colorless. Further purification by diffusion pump distillation $\left(140{ }^{\circ} \mathrm{C}\right.$, at $\left.7.0 \times 10^{-5} \mathrm{mmHg}\right)$ afforded $1.90 \mathrm{~g}(4.23$ mmol, 89\%) of analytically pure $\mathbf{1 3}$ as a colorless oil.

\section{Data for 13:}

bp: $\quad 140^{\circ} \mathrm{C}\left(7.0 \times 10^{-5} \mathrm{mmHg}, \mathrm{ABT}\right)$

${ }^{1} \mathrm{H}$ NMR: $\quad\left(500 \mathrm{MHz}, \mathrm{CDCl}_{3}\right)$

$4.88(\mathrm{~d}, J=2.0 \mathrm{~Hz}, 1 \mathrm{H}, \mathrm{CH}(2)) ; 4.25(\mathrm{dd}, J=7.3,2.0 \mathrm{~Hz}, 1 \mathrm{H}, \mathrm{CH}(3)) ; 4.15(\mathrm{dd}, J$ $=10.3,5.1 \mathrm{~Hz}, 1 \mathrm{H},(\mathrm{CHe}(6)) ; 3.97$ (sept, $J=3.7 \mathrm{~Hz}, 1 \mathrm{H},(\mathrm{SiH}(12)) ; 3.94$ (t, $J=$ $10.3 \mathrm{~Hz}, 1 \mathrm{H},(\mathrm{CHa}(6)) ; 3.93$ (dd, $J=10.1,7.0 \mathrm{~Hz}, 1 \mathrm{H}, \mathrm{CH}(4)) ; 3.74$ (dt, $J=10.3$, 
$5.1 \mathrm{~Hz}, 1 \mathrm{H}, \mathrm{CH}(5)) ; 1.06\left(\mathrm{~s}, 9 \mathrm{H},\left(\mathrm{CH}_{3}\right)_{3} \mathrm{C}(8 \mathrm{a}\right.$ or $\left.8 \mathrm{~b})\right) ; 1.00\left(\mathrm{~s}, 9 \mathrm{H},\left(\mathrm{CH}_{3}\right)_{3} \mathrm{C}(8 \mathrm{a}\right.$ or 8b)); 0.99 (t, $J=8.0 \mathrm{~Hz}, 9 \mathrm{H}, \mathrm{CH}_{3}(10)$ ); 0.69 (dq, $J=8.0,2.0 \mathrm{~Hz}, 6 \mathrm{H}, \mathrm{CH}_{2}(9)$ ); $0.158\left(\mathrm{~d}, J=3.7 \mathrm{~Hz}, 6 \mathrm{H}, \mathrm{Si}\left(\mathrm{CH}_{3}\right)_{2}(11)\right)$

${ }^{13} \mathrm{C} \mathrm{NMR}: \quad\left(126 \mathrm{MHz}, \mathrm{CDCl}_{3}\right)$

157.56 (C(1)); 115.49 (C(2)); 77.21 (C(4)); $73.11(\mathrm{C}(5)) ; 71.14$ (C(3)); 66.12 $(\mathrm{C}(6)) ; 27.44(\mathrm{C}(8 \mathrm{a}$ or $8 \mathrm{~b})) ; 26.94(\mathrm{C}(8 \mathrm{a}$ or $8 \mathrm{~b})) ; 22.72(\mathrm{C}(7 \mathrm{a}$ or $7 \mathrm{~b})) ; 19.82(\mathrm{C}(7 \mathrm{a}$ or $7 \mathrm{~b})) ; 6.84(\mathrm{C}(9)) ; 4.83(\mathrm{C}(10)) ;-5.47(\mathrm{C}(11))$

IR: (neat)

2959 (s); 2935 (s); 2860 (s); 2136 (m); $1620(\mathrm{w}) ; 1473$ (m); $1441(\mathrm{w}) ; 1386(\mathrm{w})$; 1249 (m); 1162 (s); 1130 (s); 1060 (s); 1016 (s); 897 (s); 872 (s); 826 (s); 796 (s); $770(\mathrm{~s}) ; 653(\mathrm{~m})$

MS: $\quad(\mathrm{EI}, 70 \mathrm{eV})$ $458\left(\mathrm{M}^{+}(1)\right) ; 446$ (6); 245 (17); 244 (39); 147 (30); 139 (100); 133 (14); 95 (11); 75 (14); 59 (28); 57 (15)

Opt. Rot.: $\quad[\alpha]_{\mathrm{D}}^{24}-37.8\left(\mathrm{c}=4.62, \mathrm{CH}_{3} \mathrm{Cl}\right)$

TLC: $\quad R_{f} 0.61$ (hexanes/EtOAc, 20/1) $\left[\mathrm{SiO}_{2}, \mathrm{CAM}\right]$

Analysis: $\quad \mathrm{C}_{22} \mathrm{H}_{46} \mathrm{O}_{4} \mathrm{Si}_{3}(458.85)$

Calcd: $\quad$ C, $57.59 \%$; H, $10.10 \%$

Found: $\quad$ C, $57.76 \%$; $\quad$ H, 10.29\%

\section{Preparation 1,5-Anhydro-4,6-O-[bis(1,1-dimethylethyl)silylene]-2-deoxy-1- $C$ -} (hydroxydimethylsilyl))-3-O-(triethylsilyl)-D-arabino-1-hexenitol (3) [CSR-V-60]

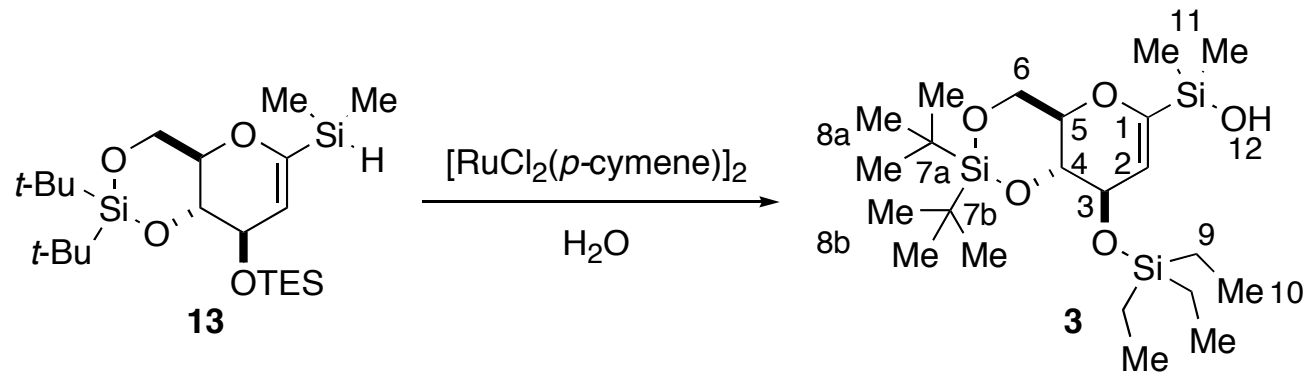

To a 10-mL, one-necked round-bottom flask, equipped with a magnetic stir-bar was charged with silane 13 (0.715 g, $1.56 \mathrm{mmol}, 1.0$ equiv) and benzene (4.0 mL). To another 50$\mathrm{mL}$, round-bottom flask, equipped with a magnetic stir-bar was added di- $\mu$-chlorobis[ $[p$ - 
cymene)chlororuthenium(II)] (0.027 g, $0.047 \mathrm{mmol}, 0.03$ equiv ) along with $\mathrm{CH}_{3} \mathrm{CN}(6.0 \mathrm{~mL})$ and benzene $2.0 \mathrm{~mL})$ followed by $\mathrm{H}_{2} \mathrm{O}(56 \mu \mathrm{L}, 3.12 \mathrm{mmol}, 2.0$ equiv). The solution of $\mathbf{1 3}$ was added drop wise by pipette and bubbling was observed. After the addition, the orange solution was stirred open to air for $1 \mathrm{~h}$ at room temperature. Over the course of the reaction the contents darkened from red to a final blood-red color. The red solution was diluted with $\mathrm{H}_{2} \mathrm{O}(30 \mathrm{~mL})$. The aqueous layer was extracted with EtOAc $(3 \times 30 \mathrm{~mL})$ and the combined organic extracts were washed with brine $(30 \mathrm{~mL})$. The contents were concentrated and immediately subjected to column chromatography (( $\left.\mathrm{SiO}_{2}, 40 \times 200 \mathrm{~mm}\right)$, hexanes/EtOAc, 15/1), which afforded $0.622 \mathrm{~g}$ (84\%) of 3 as colorless viscous oil.

Data for 3 :

bp: $\quad 145^{\circ} \mathrm{C}\left(2.0 \times 10^{-5} \mathrm{mmHg}, \mathrm{ABT}\right)$

${ }^{1} \mathrm{H}$ NMR: $\quad\left(500 \mathrm{MHz}, \mathrm{CDCl}_{3}\right)$

$4.94(\mathrm{~d}, J=2.1 \mathrm{~Hz}, 1 \mathrm{H}, \mathrm{CH}(2)) ; 4.26$ (dd, $J=7.3,2.0 \mathrm{~Hz}, 1 \mathrm{H}, \mathrm{CH}(3)) ; 4.16$ (dd, $J$ $=10.1,4.8 \mathrm{~Hz}, 1 \mathrm{H}, \mathrm{CHe}(6)) ; 3.94$ (t, $J=10.1 \mathrm{~Hz}, 1 \mathrm{H}, \mathrm{CHa}(6)$ ); 3.93 (dd, $J=$ 10.1, $7.0 \mathrm{~Hz}, 1 \mathrm{H}, \mathrm{CH}(4)) ; 3.75$ (dt, $J=10.1,5.4 \mathrm{~Hz}, 1 \mathrm{H}, \mathrm{CH}(5)) ; 1.76$ (s, $1 \mathrm{H}$, $\mathrm{SiOH}(12)) ; 1.06$ (s, 9H, $\left(\mathrm{CH}_{3}\right)_{3} \mathrm{C}(8 \mathrm{a}$ or $\left.8 \mathrm{~b})\right) ; 1.00$ (s, 9H, $\left(\mathrm{CH}_{3}\right)_{3} \mathrm{C}(8 \mathrm{a}$ or $\left.8 \mathrm{~b})\right) ; 0.99$ (t, $\left.J=7.7 \mathrm{~Hz}, 9 \mathrm{H}, \mathrm{CH}_{3}(10)\right) ; 0.69$ (dq, $\left.J=7.7,2.0 \mathrm{~Hz}, 6 \mathrm{H}, \mathrm{CH}_{2}(9)\right) ; 0.22$ (s, 6H, $\left.\mathrm{Si}\left(\mathrm{CH}_{3}\right)_{2}(11)\right)$

${ }^{13} \mathrm{C}$ NMR: $\quad\left(126 \mathrm{MHz}, \mathrm{CDCl}_{3}\right)$

$158.28(\mathrm{C}(1))$; $114.84(\mathrm{C}(2))$; $76.97(\mathrm{C}(4)) ; 73.07(\mathrm{C}(5)) ; 71.01(\mathrm{C}(3)) ; 66.13$ $(\mathrm{C}(6)) ; 27.44(\mathrm{C}(8 \mathrm{a}$ or $8 \mathrm{~b})) ; 26.94(\mathrm{C}(8 \mathrm{a}$ or $8 \mathrm{~b})) ; 22.73(\mathrm{C}(7 \mathrm{a}$ or $7 \mathrm{~b})) ; 6.84(\mathrm{C}(9))$; $4.85(\mathrm{C}(10)) ;-1.32(\mathrm{C}(11))$

IR: (neat)

3401 (s); 2994 (s); 2956 (s); 2877 (s); 1613 (m); 1459 (m); 1382 (s); 1370 (s); 1251 (s); 1168 (s); 943 (w); 891 (s); 828 (s); 782 (s); 744 (s)

MS: $\quad(\mathrm{EI}, 70 \mathrm{eV})$

$474\left(\mathrm{M}^{+}\right.$(2)); 261 (13); 260 (41); 213 (13); 177 (26); 156 (14); 155 (100); 133 (12); $115(10)$

Opt. Rot.: $\quad[\alpha]_{\mathrm{D}}^{24}-40.55\left(\mathrm{c}=0.512, \mathrm{CH}_{3} \mathrm{Cl}\right)$

TLC: $\quad R_{f} 0.22$ (hexanes/EtOAc, 10/1) $\left[\mathrm{SiO}_{2}, \mathrm{CAM}\right]$ 
Analysis: $\quad \mathrm{C}_{22} \mathrm{H}_{46} \mathrm{O}_{5} \mathrm{Si}_{3}(474.85)$

Calcd: $\quad$ C, $55.65 \% ; \quad H, 9.76 \%$

Found: $\quad$ C, $55.52 \% ; \quad H, 9.99 \%$

Preparation of 1,5-Anhydro-4,6-O-[bis(1,1-dimethylethyl)silylene]-1-C-[2',4'bis(phenylmethoxy)-6'-[(2",2"'-dimethylpropanoyl)methoxy]phenyl]-2-deoxy-3-Otriethylsilyl- $D$-arabino-1-hexenitol (14) [CSR-VI-44]

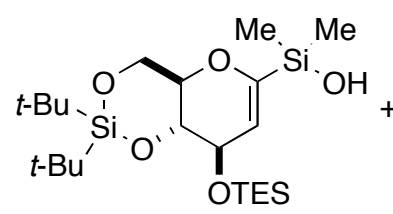

3<smiles>Oc1cc(OCc2ccccc2)cc(COc2ccccc2Br)c1I</smiles>

4

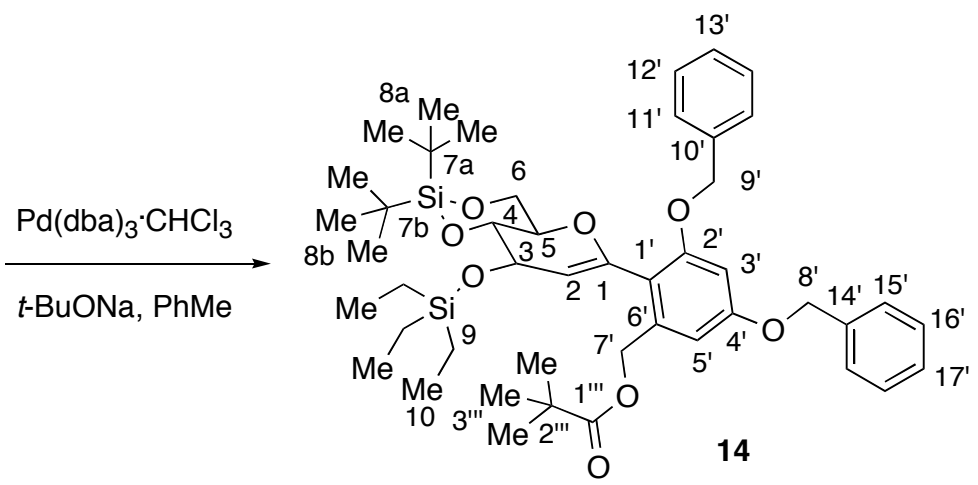

A 25-mL, one-necked, round-bottom flask containing a magnetic stir-bar was charged with aryl iodide 4 (0.559 g, $1.05 \mathrm{mmol}, 1.0$ equiv), sodium tert-butoxide (0.202 g, $2.11 \mathrm{mmol}$, 2.0 equiv) and $\mathrm{Pd}_{2}(\mathrm{dba})_{3} \cdot \mathrm{CHCl}_{3}(0.0052 \mathrm{~g}, 0.052 \mathrm{mmol}, 0.05$ equiv $)$. A water condenser fitted with an argon inlet with a rubber septum was attached and the contents of the flask were placed under an atmosphere of argon. Toluene $(3 \mathrm{~mL})$ was added and a red-purple suspension was observed. To this suspension a solution of silanol $3(0.500 \mathrm{mg}, 1.05 \mathrm{mmol}, 1.0$ equiv) in toluene $(3 \mathrm{~mL})$ under argon was added via syringe. Then the contents were stirred and heated under argon at $50{ }^{\circ} \mathrm{C}$ for $5 \mathrm{~h}$. The reaction mixture was diluted with EtOAc $(10 \mathrm{~mL})$ and $\mathrm{H}_{2} \mathrm{O}(20 \mathrm{~mL})$, transferred to a $125-\mathrm{mL}$ separatory funnel and the aqueous layer was removed. The organic layer was washed with $10 \%$ aqueous solution of 2-dimethylaminoethanethiol $(10 \mathrm{~mL})$. The aqueous extract was back-extracted with EtOAc $(3 \times 10 \mathrm{~mL})$. Then the combined organic layers were washed with brine $(15 \mathrm{~mL})$, dried $\left(\mathrm{Na}_{2} \mathrm{SO}_{4}\right)$, filtered and concentrated, initially by rotary evaporation and then by high vacuum $(0.08 \mathrm{mmHg})$, to afford a brown oil. The crude material was loaded directly onto a silica column and purified twice by column chromatography $\left(\left(\mathrm{SiO}_{2}\right.\right.$, $40 \times 200)$, hexanes/EtOAc, 10/1), then ( $\left(\mathrm{SiO}_{2}, 40 \times 200\right), \mathrm{CH}_{2} \mathrm{Cl}_{2} /$ hexanes 2/1) to afford $0.693 \mathrm{~g}$ $(82 \%)$ of $\mathbf{1 4}$ as a colorless wax. 


\section{Data for 14:}

mp: $\quad 95-97{ }^{\circ} \mathrm{C}\left(\mathrm{CH}_{2} \mathrm{Cl}_{2} /\right.$ hexanes, $\left.2 / 1\right)$

${ }^{1} \mathrm{H} \mathrm{NMR}: \quad\left(500 \mathrm{MHz}, \mathrm{CDCl}_{3}\right)$

7.28-7.45 (m, $\left.10 \mathrm{H}, \mathrm{CH}\left(15^{\prime}, 16^{\prime}, 17^{\prime}, 11^{\prime}, 12^{\prime}, 13^{\prime}\right)\right)$; $6.66(\mathrm{~d}, J=2.1 \mathrm{~Hz}, 1 \mathrm{H}$, $\mathrm{CH}\left(3^{\prime}\right.$ or $\left.\left.5^{\prime}\right)\right) ; 6.56\left(\mathrm{~d}, J=2.1 \mathrm{~Hz}, 1 \mathrm{H}, \mathrm{CH}\left(3^{\prime}\right.\right.$ or $\left.\left.5^{\prime}\right)\right) ; 5.16(\mathrm{~d}, J=13.0 \mathrm{~Hz}, 1 \mathrm{H}$, $\left.\mathrm{CH}\left(7^{\prime} \mathrm{a}\right)\right) ; 5.11$ (d, J=13.0 Hz, $\left.1 \mathrm{H}, \mathrm{CH}\left(7^{\prime} \mathrm{b}\right)\right)$; 5.04 (s, $2 \mathrm{H}, \mathrm{CH}_{2}\left(8^{\prime}\right.$ or 9')); 5.02 (s, $2 \mathrm{H}, \mathrm{CH}_{2}\left(8^{\prime}\right.$ or 9')); $4.74(\mathrm{~d}, J=2.1 \mathrm{~Hz}, 1 \mathrm{H}, \mathrm{CH}(2)) ; 4.39(\mathrm{dd}, J=7.0,2.1 \mathrm{~Hz}$, $1 \mathrm{H}, \mathrm{CH}(3)) ; 4.04-4.17(\mathrm{~m}, 2 \mathrm{H}, \mathrm{CH}(4), \mathrm{CHe}(6)) ; 3.97$ (t, $J=10.5 \mathrm{~Hz}, 1 \mathrm{H}$, $\mathrm{CHa}(6)) ; 3.94$ (dt, $J=10.54 .4 \mathrm{~Hz}, 1 \mathrm{H}, \mathrm{HC}(5)) ; 1.21$ (s, 9H, HC(3"')); 1.18 (s, 9H, $\left(\mathrm{CH}_{3}\right)_{3} \mathrm{C}(8 \mathrm{a}$ or $\left.8 \mathrm{~b})\right) ; 1.10\left(\mathrm{~s}, 9 \mathrm{H},\left(\mathrm{CH}_{3}\right)_{3} \mathrm{C}(8 \mathrm{a}\right.$ or $\left.8 \mathrm{~b})\right) ; 0.98(\mathrm{t}, J=8.0 \mathrm{~Hz}, 9 \mathrm{H}$, $\left.\mathrm{CH}_{3}(10)\right) ; 0.67$ (dq, $J=8.02 .0 \mathrm{~Hz}, 6 \mathrm{H}, \mathrm{CH}_{2}(9)$ )

${ }^{13} \mathrm{C} \mathrm{NMR}: \quad\left(126 \mathrm{MHz}, \mathrm{CDCl}_{3}\right)$

$178.17\left(\mathrm{C}\left(1^{\prime \prime \prime}\right)\right) ; 159.99\left(\mathrm{C}\left(2^{\prime}\right)\right) ; 157.90\left(\mathrm{C}\left(4^{\prime}\right)\right) ; 146.64\left(\mathrm{C}\left(1^{\prime}\right)\right) ; 137.58(\mathrm{C}(1))$; $136.90\left(\mathrm{C}\left(10^{\prime}\right.\right.$ or $\left.\left.14^{\prime}\right)\right) ; 136.56\left(\mathrm{C}\left(10^{\prime}\right.\right.$ or $\left.\left.14^{\prime}\right)\right) ; 128.65\left(\mathrm{C}\left(6^{\prime}\right.\right.$ or $11^{\prime}$ or $12^{\prime}$ or $13^{\prime}$ or $15^{\prime}$ or $16^{\prime}$ or $\left.\left.17^{\prime}\right)\right) ; 128.40\left(\mathrm{C}\left(6^{\prime}\right.\right.$ or $11^{\prime}$ or $12^{\prime}$ or $13^{\prime}$ or $15^{\prime}$ or $16^{\prime}$ or $\left.\left.17^{\prime}\right)\right) ; 128.10$ $\left(\mathrm{C}\left(6^{\prime}\right.\right.$ or $11^{\prime}$ or $12^{\prime}$ or $13^{\prime}$ or $15^{\prime}$ or $16^{\prime}$ or $\left.\left.17^{\prime}\right)\right) ; 127.76\left(\mathrm{C}\left(6^{\prime}\right.\right.$ or $11^{\prime}$ or $12^{\prime}$ or $13^{\prime}$ or $15^{\prime}$ or $16^{\prime}$ or $\left.\left.17^{\prime}\right)\right) ; 127.44\left(\mathrm{C}\left(6^{\prime}\right.\right.$ or $11^{\prime}$ or $12^{\prime}$ or $13^{\prime}$ or $1^{\prime} 5^{\prime}$ or $16^{\prime}$ or $\left.\left.17^{\prime}\right)\right) ; 126.93$ $\left(\mathrm{C}\left(6^{\prime}\right.\right.$ or $11^{\prime}$ or $12^{\prime}$ or $13^{\prime}$ or $15^{\prime}$ or $16^{\prime}$ or $\left.\left.17^{\prime}\right)\right) ; 117.50\left(\mathrm{C}\left(2^{\prime \prime \prime}\right)\right) ; 107.39(\mathrm{C}(2))$; $105.51\left(\mathrm{C}\left(3^{\prime}\right.\right.$ or $\left.\left.5^{\prime}\right)\right) ; 100.47\left(\mathrm{C}\left(3^{\prime}\right.\right.$ or $\left.\left.5^{\prime}\right)\right) ; 73.18(\mathrm{C}(5)) ; 71.41$ (C(3)); 70.32 (C(8' or $\left.\left.9^{\prime}\right)\right) ; 70.06\left(\mathrm{C}\left(8^{\prime}\right.\right.$ or $\left.\left.9^{\prime}\right)\right) ; 66.00\left(\mathrm{C}\left(7^{\prime}\right)\right) ; 63.71(\mathrm{C}(6)) ; 38.78(\mathrm{C}(4)) ; 27.43(\mathrm{C}(8 \mathrm{a}$ or $8 \mathrm{~b})) ; 27.20\left(\mathrm{C}\left(3^{\prime \prime \prime}\right)\right) ; 26.94(\mathrm{C}(8 \mathrm{a}$ or $8 \mathrm{~b})) ; 22.70(\mathrm{C}(7 \mathrm{a}$ or $7 \mathrm{~b})) ; 19.91(\mathrm{C}(7 \mathrm{a}$ or $7 b)) ; 6.85(\mathrm{C}(9)) ; 4.84(\mathrm{C}(10))$

IR: (film)

$2958(\mathrm{~m}) ; 2876(\mathrm{~m}) ; 1730(\mathrm{~m}) ; 1670(\mathrm{w}) ; 1604(\mathrm{~m}) ; 1473(\mathrm{w}) ; 1374(\mathrm{w}) ; 1322$ (w); 1281 (m); 1157 (s); 1107 (s); 1058 (m); 1014 (m); 890 (m); 844 (m); 770 (w); $696(\mathrm{w}) ; 652(\mathrm{w})$

MS: (ESI)

$\left.826\left(\left(\mathrm{M}^{+}+\mathrm{Na}\right) 14\right)\right) ; 803\left(\mathrm{M}^{+}(51)\right) ; 673(15) ; 672$ (44); 671 (100); 569 (24)

Opt. rot. : $\quad[\alpha]_{\mathrm{D}}^{24}-8.47(\mathrm{EtOH}, \mathrm{c}=0.74)$

TLC: $\quad R_{f} 0.63\left(3: 1\right.$ hexanes/EtOAc) $\left[\mathrm{SiO}_{2}, \mathrm{CAM}\right]$ 
Analysis: $\quad \mathrm{C}_{46} \mathrm{H}_{66} \mathrm{O}_{8} \mathrm{Si}_{2}(803.18)$

$\begin{array}{lll}\text { Calcd: } & \text { C, } 68.79 \% ; & \text { H, } 8.28 \% \\ \text { Found: } & \text { C, } 68.49 \% ; & \text { H, } 8.44 \%\end{array}$

Preparation of 1,5-anhydro-4,6-O-[bis(1,1-dimethylethyl)silylene]-1-C-[2',4'bis(phenylmethoxy)-6'-(methoxy)phenyl-2-deoxy-3-O-triethylsilyl-D-arabino-1-hexenitol (15) [CSR-VI-59]

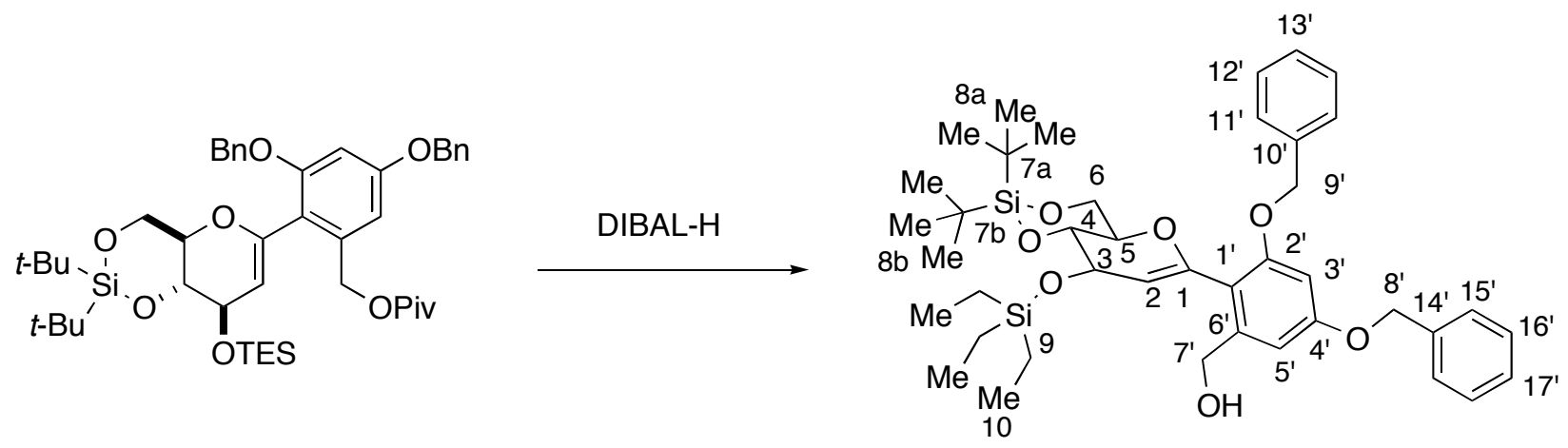

14

15

A 100-mL, one-necked, round-bottom flask fitted with an argon inlet with a septum, and a magnetic stir-bar was charged with aryl glucal $14(0.955 \mathrm{mg}, 1.18 \mathrm{mmol}, 1.0$ equiv) and $\mathrm{CH}_{2} \mathrm{Cl}_{2}(19 \mathrm{~mL})$. The solution was cooled to $-78^{\circ} \mathrm{C}$ using a 2-propanol/dry ice bath. Then DIBAL-H (1.0 M hexane, $2.5 \mathrm{~mL}, 2.48 \mathrm{mmol}, 2.1$ equiv) was added and the contents were warmed to room temperature with stirring for $0.5 \mathrm{~h}$. The solution was cooled to $0{ }^{\circ} \mathrm{C}$ (ice bath) and Celite (4 g) was added along with $\mathrm{CH}_{2} \mathrm{Cl}_{2}(5 \mathrm{~mL})$. Water $(1.2 \mathrm{~mL})$ was added very slowly ( 20 min) until the contents became gelatinous. The ice bath was removed and the contents were stirred vigorously $(\sim 10 \mathrm{~min})$ to break up the gelatinous solid. The suspension was filtered through Celite $(2 \mathrm{~g})$ and the pad was washed with EtOAc $(25 \mathrm{~mL})$. The filtrate was dried $\left(\mathrm{Na}_{2} \mathrm{SO}_{4}\right)$ decanted and concentrated to a white foam. The foam was dissolved in hexane (3.0 $\mathrm{mL})$ and concentrated to afford $0.832 \mathrm{~g}$ of $15(98 \%)$ as a white solid.

Data for 15:

mp: $\quad 50-51{ }^{\circ} \mathrm{C}$ (hexanes)

${ }^{1} \mathrm{H} \mathrm{NMR}: \quad\left(500 \mathrm{MHz}, \mathrm{CDCl}_{3}\right)$

7.31-7.43 (m, 10H, CH(15', 16', 17', 11', 12', 13')); 6.74 (d, J=2.2 Hz, 1H, CH(3' or $\left.\left.5^{\prime}\right)\right) ; 6.55\left(\mathrm{~d}, J=2.2 \mathrm{~Hz}, 1 \mathrm{H}, \mathrm{CH}\left(3^{\prime}\right.\right.$ or $\left.\left.5^{\prime}\right)\right) ; 5.07\left(\mathrm{~s}, 2 \mathrm{H}, \mathrm{CH}_{2}\left(8^{\prime}\right.\right.$ or $\left.\left.9^{\prime}\right)\right) ; 5.03(\mathrm{~s}$, 
$2 \mathrm{H}, \mathrm{CH}_{2}\left(8^{\prime}\right.$ or $\left.\left.9^{\prime}\right)\right) ; 4.76\left(\mathrm{~s}, J=2.2 \mathrm{~Hz}, 1 \mathrm{H}, \mathrm{CH}\left(7^{\prime}\right)\right) ; 4.65(\mathrm{~d}, J=6.6 \mathrm{~Hz}, 2 \mathrm{H}$, $\left.\mathrm{CH}_{2}(2)\right) ; 4.40(\mathrm{dd}, J=7.0,2.2 \mathrm{~Hz}, 1 \mathrm{H}, \mathrm{CH}(3)) ; 4.15$ (dd, $J=9.3,3.7 \mathrm{~Hz}, 1 \mathrm{H}$, $\mathrm{CHe}(6)) ; 4.08(\mathrm{dd}, J=7.0 \mathrm{~Hz}, 2.4 \mathrm{~Hz}, 1 \mathrm{H}, \mathrm{CH}(4)) ; 3.97$ (t, $J=9.3 \mathrm{~Hz}, 1 \mathrm{H}$, $\mathrm{CHa}(6)) ; 3.97$ (dt, $J=9.3,3.7 \mathrm{~Hz}, 1 \mathrm{H}, \mathrm{CH}(5))$; 1.90 (t, $J=6.6 \mathrm{~Hz}, 1 \mathrm{H}, \mathrm{br}(\mathrm{OH})$ ); $1.08\left(\mathrm{~s}, 9 \mathrm{H},\left(\mathrm{CH}_{3}\right)_{3} \mathrm{C}(8 \mathrm{a}\right.$ or $\left.8 \mathrm{~b})\right) ; 1.02\left(\mathrm{~s}, 9 \mathrm{H},\left(\mathrm{CH}_{3}\right)_{3} \mathrm{C}(8 \mathrm{a}\right.$ or $\left.8 \mathrm{~b})\right) ; 0.98(\mathrm{t}, J=8.0$ $\left.\mathrm{Hz}, 9 \mathrm{H}, \mathrm{CH}_{3}(10)\right) ; 0.66\left(\mathrm{dq}, J=8.0,2.0 \mathrm{~Hz}, 6 \mathrm{H}, \mathrm{CH}_{2}(9)\right)$

${ }^{13} \mathrm{C} \mathrm{NMR}: \quad\left(126 \mathrm{MHz}, \mathrm{CDCl}_{3}\right)$

160.29 C(2')); $\left.\left.157.72 \mathrm{C}\left(4^{\prime}\right)\right) ; 147.24 \mathrm{C}\left(1^{\prime}\right)\right)$; $\left.136.93 \mathrm{C}(1)\right)$; $136.59 \mathrm{C}\left(10^{\prime}\right.$ or $\left.\left.14^{\prime}\right)\right)$; $128.61 \mathrm{C}\left(10^{\prime}\right.$ or $\left.\left.14^{\prime}\right)\right) ; 128.39 \mathrm{C}\left(11^{\prime}\right.$ or $12^{\prime}$ or $13^{\prime}$ or $15^{\prime}$ or $16^{\prime}$ or $\left.\left.17^{\prime}\right)\right) ; 128.61$ $\mathrm{C}\left(11^{\prime}\right.$ or $12^{\prime}$ or $13^{\prime}$ or $15^{\prime}$ or $16^{\prime}$ or $\left.\left.17^{\prime}\right)\right) ; 128.08 \mathrm{C}\left(11^{\prime}\right.$ or $12^{\prime}$ or $13^{\prime}$ or $15^{\prime}$ or $16^{\prime}$ or $\left.\left.17^{\prime}\right)\right) ; 127.74 \mathrm{C}\left(11^{\prime}\right.$ or $12^{\prime}$ or $13^{\prime}$ or $15^{\prime}$ or $16^{\prime}$ or $\left.\left.17^{\prime}\right)\right)$; $127.53 \mathrm{C}\left(11^{\prime}\right.$ or $12^{\prime}$ or $13^{\prime}$ or $15^{\prime}$ or $16^{\prime}$ or $\left.\left.17^{\prime}\right)\right)$; $126.87 \mathrm{C}\left(11^{\prime}\right.$ or $12^{\prime}$ or $13^{\prime}$ or $15^{\prime}$ or $16^{\prime}$ or $\left.\left.17^{\prime}\right)\right)$; $\left.116.79 \mathrm{C}\left(6^{\prime}\right)\right)$; 107.46 C(2)); $105.50 \mathrm{C}\left(3^{\prime}\right.$ or $\left.\left.5^{\prime}\right)\right) ; 100.31 \mathrm{C}\left(3^{\prime}\right.$ or $\left.\left.5^{\prime}\right)\right)$; $\left.\left.77.18 \mathrm{C}(4)\right) ; 73.28 \mathrm{C}(3)\right)$; $71.43 \mathrm{C}(5))$; $70.35 \mathrm{C}\left(8^{\prime}\right.$ or $\left.\left.9^{\prime}\right)\right)$; $70.11 \mathrm{C}\left(8^{\prime}\right.$ or 9')); $\left.\left.65.95 \mathrm{C}(6)\right) ; 63.41 \mathrm{C}\left(7^{\prime}\right)\right) ; 27.42$ $\mathrm{C}(8 \mathrm{a}$ or $8 \mathrm{~b})) ; 26.94 \mathrm{C}(8 \mathrm{a}$ or $8 \mathrm{~b})) ; 22.73 \mathrm{C}(7 \mathrm{a}$ or $7 \mathrm{~b})) ; 26.94 \mathrm{C}(7 \mathrm{a}$ or $7 \mathrm{~b})) ; 6.85$ $\mathrm{C}(10)) ; 4.84 \mathrm{C}(9))$

IR: (film)

3436 (w); 2934 (s); 2877 (s); 2860 (w); 1670 (w); 1603 (s); 1472 (m); 1322 (m); 1157 (s); 1107 (s); $1077(\mathrm{~s}) ; 890$ (m); 826 (m); 736 (m)

MS: (ESI)

$719\left(\mathrm{M}^{+}(27)\right) ; 588(40) ; 587(100)$

Opt. rot. : $\quad[\alpha]_{D}^{24}-9.67(\mathrm{EtOH}, \mathrm{c}=1.00)$

TLC: $\quad R_{f} 0.15\left(10: 1\right.$ hexanes/EtOAc) $\left[\mathrm{SiO}_{2}, \mathrm{UV}\right]$

Analysis: $\quad \mathrm{C}_{41} \mathrm{H}_{58} \mathrm{O}_{7} \mathrm{Si}_{2}(719.07)$

Calcd: $\quad$ C, $68.48 \%$; H, $8.13 \%$

Found: $\quad \mathrm{C}, 68.17 \% ; \quad \mathrm{H}, 8.02 \%$ 
Preparation of $\left(1 S, 4^{\prime} a R, 7^{\prime} R, 8^{\prime} R, 8^{\prime} a R\right)-S p i r o\left[i s o b e n z o f u r a n-1(3 H), 6^{\prime}\left(4^{\prime} H\right)-p y r a n o[3,2-\right.$ d] $[1,3,2]$ dioxasilin]-7'-ol, bis(phenylmethoxy)-8'- $\quad$ triethylsilyl $(16-\alpha) \quad$ and $(1 R$,
Spiro[isobenzofuran-1(3H),6' $\left(4^{\prime} H\right)$-pyrano[3,2-d][1,3,2]dioxasilin]-7'-ol, 2',2'-bis(1,1-dimethylethyl)-4'a,7',8',8'a-tetrahydro-5,7,dimethylethyl)-4'a,7',8',8'a-tetrahydro-5,7,- bis(phenylmethoxy)-8' - triethylsilyl (16- $\beta$ ) [CSR-VIII-76]

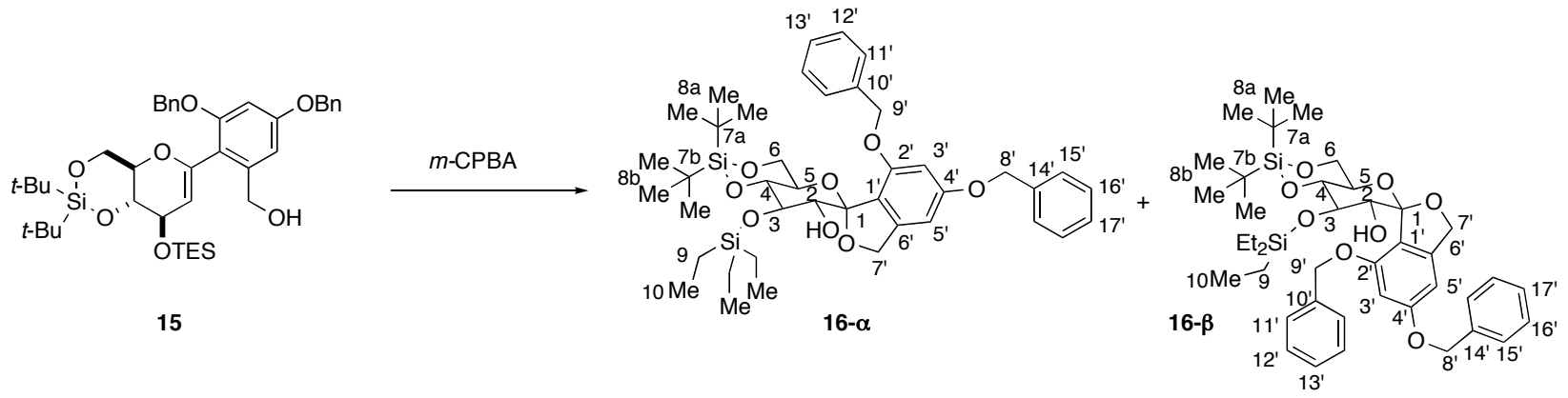

A 250-mL, one-necked, round-bottom flask fitted with an argon inlet with a septum, and a magnetic stir-bar was charged with 15 (0.930 mg, $1.29 \mathrm{mmol}, 1.0$ equiv), $\mathrm{CH}_{2} \mathrm{Cl}_{2}$ (31 mL), and $\mathrm{NaHCO}_{3}\left(0.325 \mathrm{~g}, 3.87 \mathrm{mmol}, 3.0\right.$ equiv). Then the solution was cooled to $0{ }^{\circ} \mathrm{C}$ using an ice bath. To a separate 25-mL, one-necked, conical flask, fitted with an argon inlet with a septum and magnetic stir-bar was added washed $m$-CPBA (98\%, $0.267 \mathrm{~g}, 1.55 \mathrm{mmol}, 1.2$ equiv) along with $\mathrm{CH}_{2} \mathrm{Cl}_{2}(10 \mathrm{~mL})$. This solution was transferred via cannula into the solution of $\mathbf{1 5}$. The contents were stirred at $0{ }^{\circ} \mathrm{C}$ for $5 \mathrm{~min}$, and warmed to room temperature and stirred at this temperature for $3 \mathrm{~h}$. To the cloudy mixture was added $\mathrm{H}_{2} \mathrm{O}(20 \mathrm{~mL})$ and the aqueous extract was back-extracted with $\mathrm{CH}_{2} \mathrm{Cl}_{2}(3 \times 15 \mathrm{~mL})$, the organic layers combined, washed with brine $(15 \mathrm{~mL})$, dried $\left(\mathrm{Na}_{2} \mathrm{SO}_{4}\right)$, filtered and concentrated to afford a white foam. The crude reaction mixture was analyzed by ${ }^{1} \mathrm{H}-\mathrm{NMR}$ to afford a $5: 1$ ratio of anomers $(\mathbf{1 6}-\boldsymbol{\alpha}: \mathbf{1 6}-\boldsymbol{\beta})$. The sample was analyzed using dry and non-acidic $\mathrm{CDCl}_{3}$ (freshly percolated through Brockmann Act I basic alumina and stored over $3 \AA$ molecular sieves prior to use). The crude material was purified by column chromatography ( $\left(\mathrm{SiO}_{2}, 40 \times 180\right)$, hexanes/EtOAc, $\left.7 / 1\right)$ to afford a colorless wax (m.p. $56-61{ }^{\circ} \mathrm{C}$ for $\mathbf{1 6}-\boldsymbol{\alpha}$ ) of separated isomers. The separated isomers were recrystallized from hexanes (15 mL for $\mathbf{1 6}-\boldsymbol{\alpha}, 5 \mathrm{~mL}$ for $\mathbf{1 6}-\beta)$ to afford $0.733 \mathrm{~g}(77 \%)$ of $\mathbf{1 6}-\alpha$ as a white, cubic solid and $0.142 \mathrm{~g}(15 \%)$ of $\mathbf{1 6 - \beta}$ as a white powder.

\section{Data for 16- $\alpha$ :}

mp: $\quad 130-131{ }^{\circ} \mathrm{C}$ (hexane) 
${ }^{1}$ H NMR: $\quad\left(500 \mathrm{MHz}, \mathrm{CDCl}_{3}\right)$

7.29-7.39 (m, 10H, CH(15', 16', 17', 11', 12', 13')); 6.47 (d, $J=1.7 \mathrm{~Hz}, 1 \mathrm{H}$, $\left.\mathrm{CH}\left(3^{\prime}\right)\right) ; 6.40\left(\mathrm{~d}, J=1.7 \mathrm{~Hz}, 1 \mathrm{H}, \mathrm{CH}\left(5^{\prime}\right)\right) ; 5.16\left(\mathrm{~d}, J=12.4 \mathrm{~Hz}, 1 \mathrm{H}, \mathrm{CH}\left(7^{\prime} \mathrm{a}\right)\right) ; 5.11$ (s, $2 \mathrm{H}, \mathrm{CH}_{2}\left(8^{\prime}\right.$ or $\left.\left.9^{\prime}\right)\right) ; 5.07\left(\mathrm{~d}, J=12.4 \mathrm{~Hz}, 1 \mathrm{H}, \mathrm{CH}\left(7^{\prime} \mathrm{b}\right)\right) ; 5.00$ (s, $2 \mathrm{H}, \mathrm{CH}_{2}\left(8^{\prime}\right.$ or 9')); 4.32 (dd, $J=10.2,7.3 \mathrm{~Hz}, 1 \mathrm{H}, \mathrm{CH}(2)) ; 4.13$ (dd, $J=10.2,4.4 \mathrm{~Hz}, 1 \mathrm{H}$, $\mathrm{CH}(6 \mathrm{e})$ ); 3.96 (dd, $J=10.2,4.4 \mathrm{~Hz}, 1 \mathrm{H}, \mathrm{CH}(5)) ; 3.88$ (t, $J=10.2 \mathrm{~Hz}, 1 \mathrm{H}, \mathrm{CH}(4))$; $3.84(\mathrm{t}, J=9.8 \mathrm{~Hz}, 1 \mathrm{H}, \mathrm{CH}(3)) ; 3.78$ (t, $J=10.2 \mathrm{~Hz}, 1 \mathrm{H}, \mathrm{CH}(6 \mathrm{a})) ; 1.84$ (d, $J=7.3$ $\mathrm{Hz}, 1 \mathrm{H}, \mathrm{br}(\mathrm{OH})) ; 1.08$ (s, 9H, $\left(\mathrm{CH}_{3}\right)_{3} \mathrm{C}(8 \mathrm{a}$ or $\left.8 \mathrm{~b})\right) ; 1.04\left(\mathrm{~s}, 9 \mathrm{H},\left(\mathrm{CH}_{3}\right)_{3} \mathrm{C}(8 \mathrm{a}\right.$ or $8 \mathrm{~b})$ ); $0.99\left(\mathrm{t}, J=8.0 \mathrm{~Hz}, 9 \mathrm{H}, \mathrm{CH}_{3}(10)\right) ; 0.70\left(\mathrm{dq}, J=8.0,2.0 \mathrm{~Hz}, 6 \mathrm{H}, \mathrm{CH}_{2}(9)\right)$

${ }^{13} \mathrm{C} \mathrm{NMR}: \quad\left(126 \mathrm{MHz}, \mathrm{CDCl}_{3}\right)$

162.06 $\left.\mathrm{C}\left(2^{\prime}\right)\right)$; $\left.154.73 \mathrm{C}\left(4^{\prime}\right)\right)$; $\left.143.41 \mathrm{C}\left(1^{\prime}\right)\right)$; $136.79 \mathrm{C}\left(10^{\prime}\right.$ or $\left.\left.14^{\prime}\right)\right)$; $136.59 \mathrm{C}\left(10^{\prime}\right.$ or $\left.\left.14^{\prime}\right)\right) ; 128.65 \mathrm{C}\left(11^{\prime}\right.$ or $12^{\prime}$ or $13^{\prime}$ or $15^{\prime}$ or $16^{\prime}$ or $\left.\left.17^{\prime}\right)\right)$; $128.46 \mathrm{C}\left(11^{\prime}\right.$ or $12^{\prime}$ or $13^{\prime}$ or $1^{\prime}$ or $16^{\prime}$ or $\left.\left.17^{\prime}\right)\right)$; $128.09 \mathrm{C}\left(11^{\prime}\right.$ or $12^{\prime}$ or $13^{\prime}$ or $15^{\prime}$ or $16^{\prime}$ or $\left.\left.17^{\prime}\right)\right)$; $127.77 \mathrm{C}\left(11^{\prime}\right.$ or $12^{\prime}$ or $13^{\prime}$ or $15^{\prime}$ or $16^{\prime}$ or $\left.\left.1^{\prime}\right)\right)$; $127.41 \mathrm{C}\left(11^{\prime}\right.$ or $12^{\prime}$ or $13^{\prime}$ or $15^{\prime}$ or $16^{\prime}$ or $\left.17^{\prime}\right)$ ); $126.67 \mathrm{C}\left(11^{\prime}\right.$ or $12^{\prime}$ or $13^{\prime}$ or $15^{\prime}$ or $16^{\prime}$ or $\left.\left.17^{\prime}\right)\right)$; $\left.118.17 \mathrm{C}\left(6^{\prime}\right)\right)$; $\left.110.85 \mathrm{C}(1)\right)$; 100.48 $\left.\left.\mathrm{C}\left(3^{\prime}\right)\right) ; 98.36 \mathrm{C}\left(5^{\prime}\right)\right)$; $\left.\left.\left.77.78 \mathrm{C}(4)\right) ; 76.64 \mathrm{C}(2)\right) ; 73.22 \mathrm{C}(5)\right)$; $\left.73.16 \mathrm{C}(3)\right)$; $70.37 \mathrm{C}\left(8^{\prime}\right.$ or $\left.\left.9^{\prime}\right)\right)$; $69.69 \mathrm{C}\left(8^{\prime}\right.$ or $\left.\left.9^{\prime}\right)\right)$; $\left.68.97 \mathrm{C}\left(7^{\prime}\right)\right)$; $\left.67.13 \mathrm{C}(6)\right)$; $27.58 \mathrm{C}(8 \mathrm{a}$ or $8 b)) ; 27.06 \mathrm{C}(8 \mathrm{a}$ or $8 \mathrm{~b})) ; 22.82 \mathrm{C}(7 \mathrm{a}$ or $7 \mathrm{~b})) ; 19.93 \mathrm{C}(7 \mathrm{a}$ or $7 \mathrm{~b})) ; 6.95 \mathrm{C}(10))$; $5.16 \mathrm{C}(9))$

IR: (film)

3569 (w); 3033 (w); 2950 (s); 2934 (s); 2875 (s); 2860 (s); 1728 (w); 1610 (s); 1498 (m); 1444 (m); 1378 (m); 1300 (m); 1160 (s); 1073 (s); 1021 (s); 965 (m); $828(\mathrm{~s}) ; 736(\mathrm{~s}) ; 696(\mathrm{~m})$

MS: (ESI)

735 (M+ (25); 604 (27); 603 (100); 357 (21); 213 (15); 201 (14); 177 (28)

Opt. rot. : $\quad[\alpha]_{D}^{24}-10.09(\mathrm{EtOH}, \mathrm{c}=0.85)$

TLC: $\quad R_{f} 0.31$ (7:1 hexanes/EtOAc) $\left[\mathrm{SiO}_{2}, \mathrm{CAM}\right]$

Analysis: $\quad \mathrm{C}_{41} \mathrm{H}_{58} \mathrm{O}_{8} \mathrm{Si}_{2}(735.07)$

Calcd: $\quad$ C, $66.99 \% ; \quad H, 7.95 \%$

Found: $\quad$ C, $67.02 \% ; \quad H, 8.17 \%$ 


\section{Data for 16- $\beta$ :}

mp: $\quad 120-122^{\circ} \mathrm{C}$ (hexane)

${ }^{1} \mathrm{H} \mathrm{NMR}: \quad\left(500 \mathrm{MHz}, \mathrm{CDCl}_{3}\right)$

7.26-7.38 (m, 10H, CH(15', 16', 17', 11', 12', 13')); $6.42(\mathrm{~d}, J=2.0 \mathrm{~Hz}, 1 \mathrm{H}$, $\left.\mathrm{CH}\left(3^{\prime}\right)\right) ; 6.32\left(\mathrm{~d}, J=2.0 \mathrm{~Hz}, 1 \mathrm{H}, \mathrm{CH}\left(5^{\prime}\right)\right) ; 5.31$ (d, $J=14.2 \mathrm{~Hz}, 1 \mathrm{H}, \mathrm{CH}\left(8^{\prime} \mathrm{a}\right.$ or 9'a)); $5.26\left(\mathrm{~d}, J=14.2 \mathrm{~Hz}, 1 \mathrm{H}, \mathrm{CH}\left(8^{\prime} \mathrm{a}\right.\right.$ or 9'a)); $5.14(\mathrm{~d}, J=12.8 \mathrm{~Hz}, 1 \mathrm{H}$, $\left.\mathrm{CH}\left(7^{\prime} \mathrm{a}\right)\right) ; 4.92\left(\mathrm{~d}, J=12.8 \mathrm{~Hz}, 1 \mathrm{H}, \mathrm{CH}\left(7^{\prime} \mathrm{b}\right)\right) ; 4.90$ (s, $2 \mathrm{H}, \mathrm{CH}_{2}\left(8^{\prime}\right.$ or 9')); 4.51 (t, $J=8.2 \mathrm{~Hz}, 1 \mathrm{H}, \mathrm{CH}(3)) ; 4.10(\mathrm{dt}, J=9.4,4.3 \mathrm{~Hz}, 1 \mathrm{H}, \mathrm{CH}(5)) ; 4.06$ (dd, $J=9.4$, $4.3 \mathrm{~Hz}, 1 \mathrm{H}, \mathrm{CH}(6 \mathrm{e})$ ); 3.97 (dd, $J=9.4,8.2 \mathrm{~Hz}, 1 \mathrm{H}, \mathrm{CH}(4)) ; 3.92$ (t, $J=8.2,3.1$ $\mathrm{Hz}, 1 \mathrm{H}, \mathrm{CH}(2)) ; 3.86$ (t (br), $J=9.4 \mathrm{~Hz}, 1 \mathrm{H}, \mathrm{CH}(6 \mathrm{a})$ ); 2.56 (d, $3.1 \mathrm{~Hz} 1 \mathrm{H}$, $(\mathrm{brOH})) ; 1.06\left(\mathrm{~s}, 9 \mathrm{H},\left(\mathrm{CH}_{3}\right)_{3} \mathrm{C}(8 \mathrm{a}\right.$ or $\left.8 \mathrm{~b})\right) ; 0.98\left(\mathrm{~s}, 9 \mathrm{H},\left(\mathrm{CH}_{3}\right)_{3} \mathrm{C}(8 \mathrm{a}\right.$ or $\left.8 \mathrm{~b})\right) ; 0.94(\mathrm{t}$, $\left.J=8.1 \mathrm{~Hz}, 9 \mathrm{H}, \mathrm{CH}_{3}(10)\right) ; 0.58-0.75\left(\mathrm{~m}, 6 \mathrm{H}, \mathrm{CH}_{2}(9)\right)$

${ }^{13} \mathrm{C} \mathrm{NMR}: \quad\left(126 \mathrm{MHz}, \mathrm{CDCl}_{3}\right)$

$161.36\left(\mathrm{C}\left(2^{\prime}\right)\right) ; 153.79\left(\mathrm{C}\left(4^{\prime}\right)\right) ; 145.60\left(\mathrm{C}\left(1^{\prime}\right)\right) ; 136.37\left(\mathrm{C}\left(10^{\prime}\right.\right.$ or $\left.\left.14^{\prime}\right)\right) ; 136.15$ $\left(\mathrm{C}\left(10^{\prime}\right.\right.$ or $\left.\left.14^{\prime}\right)\right) ; 128.74\left(\mathrm{C}\left(11^{\prime}\right.\right.$ or $12^{\prime}$ or $13^{\prime}$ or $15^{\prime}$ or $16^{\prime}$ or $\left.\left.17^{\prime}\right)\right) ; 128.64\left(\mathrm{C}\left(11^{\prime}\right.\right.$ or $12^{\prime}$ or $13^{\prime}$ or $15^{\prime}$ or $16^{\prime}$ or $\left.\left.17^{\prime}\right)\right)$; $128.15\left(\mathrm{C}\left(11^{\prime}\right.\right.$ or $12^{\prime}$ or $13^{\prime}$ or $15^{\prime}$ or $16^{\prime}$ or $\left.\left.17^{\prime}\right)\right)$; $127.73\left(\mathrm{C}\left(11^{\prime}\right.\right.$ or $12^{\prime}$ or $13^{\prime}$ or $15^{\prime}$ or $16^{\prime}$ or $\left.\left.17^{\prime}\right)\right) ; 127.47\left(\mathrm{C}\left(11^{\prime}\right.\right.$ or $12^{\prime}$ or $13^{\prime}$ or $15^{\prime}$ or $16^{\prime}$ or $\left.\left.17^{\prime}\right)\right)$; $125.92\left(\mathrm{C}\left(11^{\prime}\right.\right.$ or $12^{\prime}$ or $13^{\prime}$ or $15^{\prime}$ or $16^{\prime}$ or $\left.\left.17^{\prime}\right)\right) ; 118.48\left(\mathrm{C}\left(6^{\prime}\right)\right)$; 113.03 (C(1)); 100.59 (C(3')); 99.33 (C(5')); 78.01 (C(4)); 77.80 (C(2)); 76.91 $(\mathrm{C}(5)) ; 71.31(\mathrm{C}(3)) ; 70.30\left(\mathrm{C}\left(8^{\prime}\right.\right.$ or $\left.\left.9^{\prime}\right)\right) ; 69.64\left(\mathrm{C}\left(8^{\prime}\right.\right.$ or $\left.\left.9^{\prime}\right)\right) ; 68.77\left(\mathrm{C}\left(7^{\prime}\right)\right) ; 67.22$ $(\mathrm{C}(6)) ; 27.57(\mathrm{C}(8 \mathrm{a}$ or $8 \mathrm{~b})) ; 26.99(\mathrm{C}(8 \mathrm{a}$ or $8 \mathrm{~b})) ; 22.74(\mathrm{C}(7 \mathrm{a}$ or $7 \mathrm{~b})) ; 19.81(\mathrm{C}(7 \mathrm{a}$ or $7 \mathrm{~b})) ; 6.89(\mathrm{C}(10)) ; 5.07(\mathrm{C}(9))$

IR: (film)

3480 (w); 3065 (w); 2934 (s); 2875 (s); 2860 (s); 1612 (s); 1597 (s); 1471 (m); $1385(\mathrm{w}) ; 1339(\mathrm{w}) ; 1296(\mathrm{w}) ; 1210(\mathrm{w}) ; 1148$ (s); 1089 (s); 1043 (s); 836 (s); 773 (s); $736(\mathrm{~s})$

MS: (ESI) $735\left(\mathrm{M}^{+}\right.$(22)); 621 (100); 603 (5); 460 (3)

Opt. rot. : $\quad[\alpha]_{D}^{24} 6.80(\mathrm{EtOH}, \mathrm{c}=0.10)$

TLC: $\quad R_{f} 0.15\left(10: 1\right.$ hexanes/EtOAc) $\left[\mathrm{SiO}_{2}, \mathrm{CAM}\right]$ 
HRMS: $\quad \mathrm{C}_{41} \mathrm{H}_{58} \mathrm{O}_{8} \mathrm{Si}_{2}(735.07)$

Calcd: $\quad 735.3749$

Found: $\quad 735.3782$

\section{Isomerization of 16- $\beta$ to $16-\alpha$ [CSR-IX-p13]}

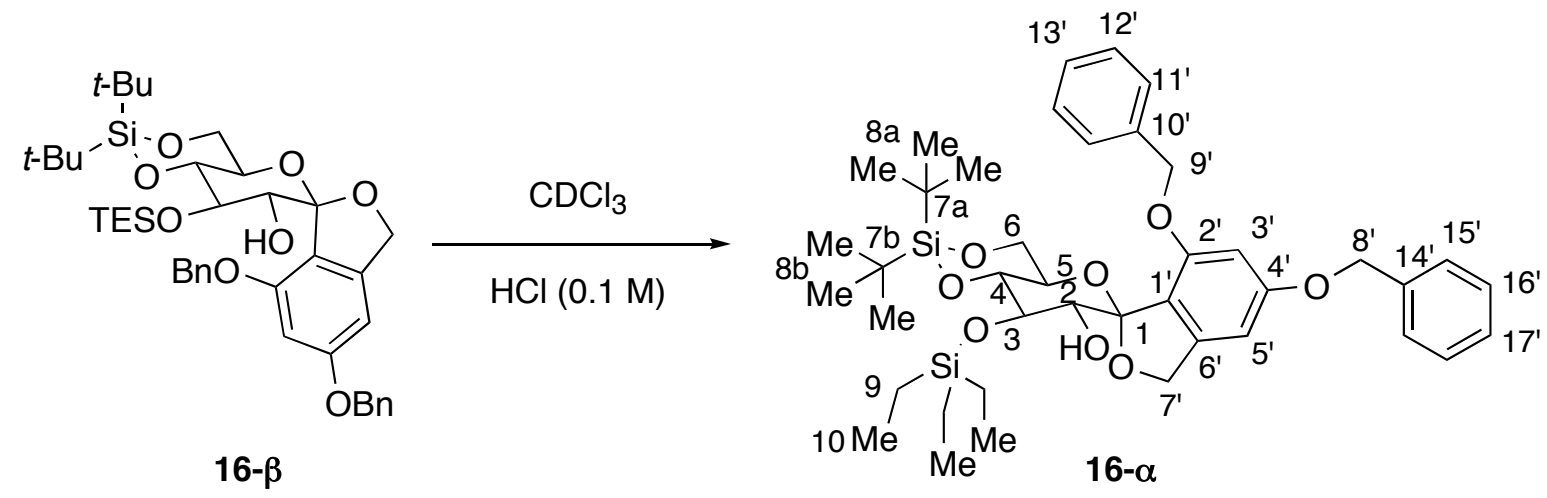

A solution of 16- $\beta(0.026 \mathrm{~g}, 0.035 \mathrm{mmol})$ in $\mathrm{CDCl}_{3}(0.7 \mathrm{~mL}$, containing $\mathrm{HCl}(0.1 \mathrm{M}$, titrated)) was analyzed by ${ }^{1} \mathrm{H}-\mathrm{NMR}$. Complete epimerization to 16- $\alpha$ was observed after $12 \mathrm{~h}$ at $20{ }^{\circ} \mathrm{C}$. After NMR analysis the contents were diluted with $\mathrm{CH}_{2} \mathrm{Cl}_{2}(0.1 \mathrm{~mL})$ and subjected to column chromatography $\left(\left(\mathrm{SiO}_{2}, 10 \times 50 \mathrm{~mm}\right)\right.$, hexanes/EtOAc, $\left.7 / 1\right)$ to afford $0.025 \mathrm{~g}(96 \%)$ of 16- $\alpha$.

Data for 16- $\alpha$ :

${ }^{1}$ H NMR: $\quad\left(500 \mathrm{MHz}, \mathrm{CDCl}_{3}\right)$

7.29-7.39 (m, 10H, CH(15', 16', 17', 11', 12', 13')); $6.47(\mathrm{~d}, J=1.7 \mathrm{~Hz}, 1 \mathrm{H}$, $\left.\mathrm{CH}\left(3^{\prime}\right)\right) ; 6.40\left(\mathrm{~d}, J=1.7 \mathrm{~Hz}, 1 \mathrm{H}, \mathrm{CH}\left(5^{\prime}\right)\right) ; 5.16\left(\mathrm{~d}, J=12.4 \mathrm{~Hz}, 1 \mathrm{H}, \mathrm{CH}\left(7^{\prime} \mathrm{a}\right)\right) ; 5.11$ (s, $2 \mathrm{H}, \mathrm{CH}_{2}\left(8^{\prime}\right.$ or $\left.\left.9^{\prime}\right)\right) ; 5.07$ (d, $\left.J=12.4 \mathrm{~Hz}, 1 \mathrm{H}, \mathrm{CH}\left(7^{\prime} \mathrm{b}\right)\right) ; 5.00$ (s, $2 \mathrm{H}, \mathrm{CH}_{2}\left(8^{\prime}\right.$ or 9')); $4.32(\mathrm{dd}, J=10.2,7.3 \mathrm{~Hz}, 1 \mathrm{H}, \mathrm{CH}(2)) ; 4.13(\mathrm{dd}, J=10.2,4.4 \mathrm{~Hz}, 1 \mathrm{H}$, $\mathrm{CH}(6 \mathrm{e})) ; 3.96$ (dd, $J=10.2,4.4 \mathrm{~Hz}, 1 \mathrm{H}, \mathrm{CH}(5)) ; 3.88$ (t, $J=10.2 \mathrm{~Hz}, 1 \mathrm{H}, \mathrm{CH}(4))$; 3.84 (t, $J=9.8 \mathrm{~Hz}, 1 \mathrm{H}, \mathrm{CH}(3)) ; 3.78$ (t, $J=10.2 \mathrm{~Hz}, 1 \mathrm{H}, \mathrm{CH}(6 \mathrm{a})) ; 1.84$ (d, $J=7.3$ $\mathrm{Hz}, 1 \mathrm{H}, \mathrm{br}(\mathrm{OH})) ; 1.08$ (s, 9H, $\left(\mathrm{CH}_{3}\right)_{3} \mathrm{C}(8 \mathrm{a}$ or $8 \mathrm{~b})$ ); 1.04 (s, $9 \mathrm{H},\left(\mathrm{CH}_{3}\right)_{3} \mathrm{C}(8 \mathrm{a}$ or $8 \mathrm{~b})$ ); 0.99 (t, $\left.J=8.0 \mathrm{~Hz}, 9 \mathrm{H}, \mathrm{CH}_{3}(10)\right) ; 0.70$ (dq, $\left.J=8.0,2.0 \mathrm{~Hz}, 6 \mathrm{H}, \mathrm{CH}_{2}(9)\right)$ 
Preparation of $\left(1 R, 4^{\prime} \mathrm{a} R, 7^{\prime} R, 8^{\prime} R, 8^{\prime} \mathrm{a} R\right)-\mathrm{Spiro}$ [isobenzofuran-1(3H),6'(4'H)-pyrano[3,2d] [1,3,2]dioxasilin]-7'-ol, $\quad$ 2',2'-bis(1,1-dimethylethyl)-4'a,7',8', $\quad$ ' a-tetrahydro-5,7,bis(phenylmethoxy)-8'- triethylsilyl-9'-(2-trimethylsilylethoxycarbonyl) (17) [CSR-VIII-35]

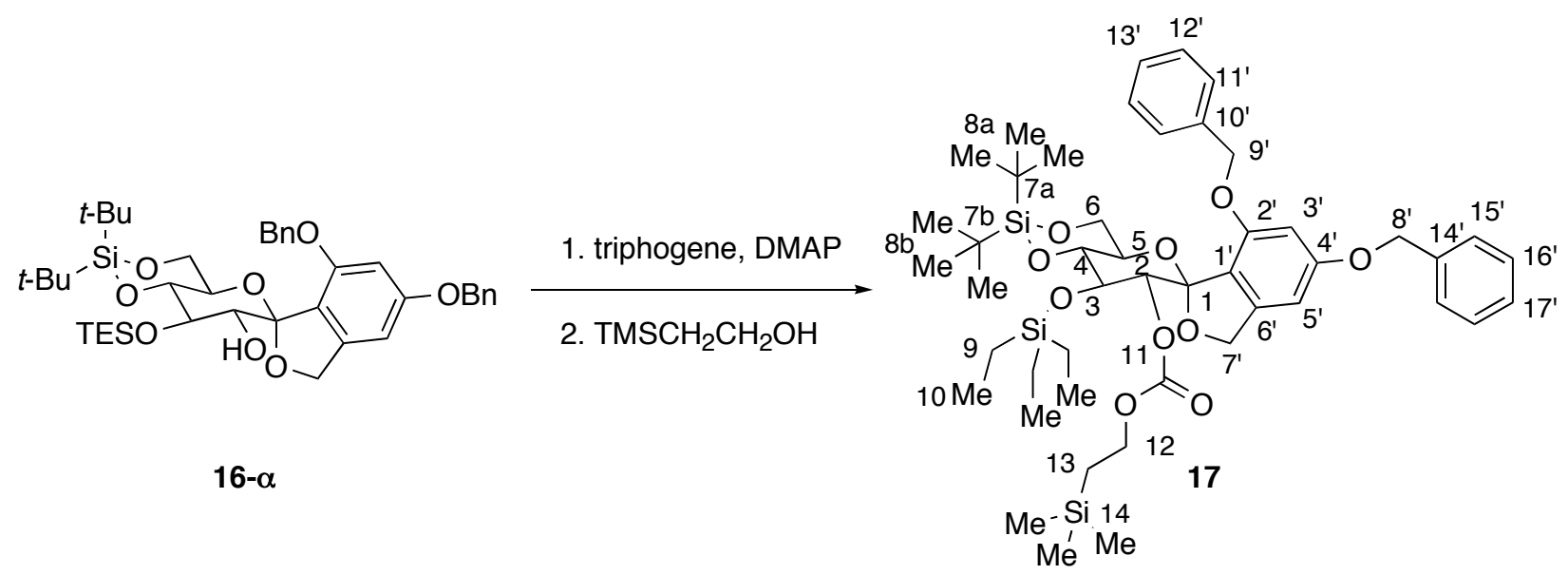

To a 25-mL Schlenk flask, equipped with a magnetic stir-bar was added triphosgene ( $0.197 \mathrm{~g}, 0.73 \mathrm{mmol}, 0.67$ equiv) and the flask was sealed with a septum and purged with argon. Then $\mathrm{CHCl}_{3}(1.0 \mathrm{~mL})$ was added and the contents were cooled in a 2-propanol bath to $-56{ }^{\circ} \mathrm{C}$ using a Cryocool to maintain the temperature. Then to a 5-mL conical flask, equipped with a magnetic stir-bar, argon inlet with septum, DMAP (0.266 g, $2.18 \mathrm{mmol}, 2.0$ equiv) was added and dissolved in $\mathrm{CHCl}_{3}(1.0 \mathrm{~mL})$. The solution of DMAP was also cooled to $-56{ }^{\circ} \mathrm{C}$. The DMAP solution was added drop wise ( $\sim 3 \mathrm{~min})$ to the solution of triphosgene. A light-yellow precipitate formed upon the addition of DMAP. The contents were warmed to room temperature and stirred at this temperature for $1 \mathrm{~h}$, where a light yellow solution was observed. To a separate 10-mL conical flask, equipped with a magnetic stir-bar and an argon inlet with a septum, spiroketal 16- $\alpha$ (0.803 g, $1.09 \mathrm{mmol}, 1.0$ equiv) was added, followed by $\mathrm{CHCl}_{3}(2.5 \mathrm{~mL})$ and $i-\operatorname{Pr}_{2} \mathrm{NEt}$ (1.33 mL, $7.63 \mathrm{mmol}, 7.0$ equiv). The Schlenk flask was once again cooled to $-56{ }^{\circ} \mathrm{C}$ and the solution of 16- $\alpha$ was added drop wise via cannula into the Schlenk flask. The flask containing 16- $\alpha$ was then rinsed with $\mathrm{CHCl}_{3}(900 \mu \mathrm{L})$. After the addition the yellow mixture was stirred for $10 \mathrm{~min}$ at $-56^{\circ} \mathrm{C}$ and then warmed to room temperature. Upon warming the contents became a yellow solution, which eventually became a yellow mixture. The mixture was stirred at room temperature for an additional $2 \mathrm{~h}$. Then 2-(trimethylsilyl)ethanol (641 $\mu \mathrm{L}, 4.45 \mathrm{mmol}, 4.1$ equiv) was added and the mixture became a yellow solution. This solution was stirred for $12 \mathrm{~h}$ at room 
temperature. Then water $(30 \mathrm{~mL})$ was and the contents were transferred to a $125-\mathrm{mL}$ separatory funnel and the aqueous layer was extracted. The aqueous layer was extracted with EtOAc $(3 \mathrm{x}$ $30 \mathrm{~mL})$. The combined organic extracts were washed with brine $(1 \times 30 \mathrm{~mL})$, dried $\left(\mathrm{Na}_{2} \mathrm{SO}_{4}\right)$, filtered and concentrated to a light yellow oil. The crude product was purified by column chromatography (( $\left.\mathrm{SiO}_{2}, 50 \times 120 \mathrm{~mm}\right)$, hexanes/EtOAc, 20/1 to 10/1) to afford $0.879 \mathrm{~g} \mathrm{(92 \% )} \mathrm{of}$ 17 as a white foam.

\section{Data for 17:}

mp: $\quad 53-55^{\circ} \mathrm{C}(10 / 1$ hexanes/EtOAc $)$

${ }^{1}$ H NMR: $\quad\left(500 \mathrm{MHz}, \mathrm{CDCl}_{3}\right.$ )

7.29-7.47 (m, 10H, CH(15', 16', 17', 11', 12', 13')); $6.43(\mathrm{~d}, J=1.7 \mathrm{~Hz}, 1 \mathrm{H}$, $\left.\mathrm{CH}\left(3^{\prime}\right)\right) ; 6.35\left(\mathrm{~d}, J=1.7 \mathrm{~Hz}, 1 \mathrm{H}, \mathrm{CH}\left(5^{\prime}\right)\right) ; 5.47(\mathrm{~d}, J=10.0 \mathrm{~Hz}, 1 \mathrm{H}, \mathrm{CH}(2)) ; 5.13$ $\left(\mathrm{d}, J=12.0 \mathrm{~Hz}, 2 \mathrm{H}, \mathrm{CH}_{2}\left(7 \mathrm{a}^{\prime}\right)\right) ; 5.12\left(\mathrm{~d}, J=12.0 \mathrm{~Hz}, 1 \mathrm{H}, \mathrm{CH}\left(8 \mathrm{a}^{\prime}\right.\right.$ or $\left.\left.9 \mathrm{a}^{\prime}\right)\right) ; 5.09$ (d, $J=12.0 \mathrm{~Hz}, 1 \mathrm{H}, \mathrm{CH}\left(8 \mathrm{~b}^{\prime}\right.$ or $\left.\left.9 \mathrm{~b}^{\prime}\right)\right) ; 5.08\left(\mathrm{~d}, J=12.0 \mathrm{~Hz}, 1 \mathrm{H}, \mathrm{CH}\left(7 \mathrm{~b}^{\prime}\right)\right) ; 4.98$ (s, $2 \mathrm{H}$, $\mathrm{CH}_{2}\left(8 \mathrm{~b}^{\prime}\right.$ or $\left.9 \mathrm{~b}^{\prime}\right)$ ); 4.32 (dd, $\left.J=10.2,7.3 \mathrm{~Hz}, 1 \mathrm{H}, \mathrm{CH}(5)\right) ; 4.14$ (dd, $J=10.0,5.0$ $\mathrm{Hz}, 1 \mathrm{H}, \mathrm{CH}(6 \mathrm{e})) ; 4.04$ (t, $J=9.0 \mathrm{~Hz}, 1 \mathrm{H}, \mathrm{CH}(4)) ; 3.98$ (dt, $J=7.8,1.2 \mathrm{~Hz}, 2 \mathrm{H}$, $\left.\mathrm{CH}_{2}(12)\right) ; 3.85$ (t, $J=10.0 \mathrm{~Hz}, 1 \mathrm{H}, \mathrm{CH}(6 \mathrm{a})$ ); 3.82 (t, $\left.J=9.5 \mathrm{~Hz}, 1 \mathrm{H}, \mathrm{CH}(3)\right) ; 1.07$ (s, 9H, $\mathrm{C}\left(\mathrm{CH}_{3}\right)_{3}(8 \mathrm{a}$ or $8 \mathrm{~b})$ ); 1.03 (s, $9 \mathrm{H}, \mathrm{C}\left(\mathrm{CH}_{3}\right)_{3}(8 \mathrm{a}$ or $8 \mathrm{~b})$ ); 0.96 (t, $J=8.0 \mathrm{~Hz}$, 9H, $\mathrm{CH}_{3}(10)$ ); 0.82 (dt, $J=8.0,2.6 \mathrm{~Hz}, 2 \mathrm{H}, \mathrm{CH}_{2}(13)$ ); 0.66 (dq, $J=8.0,2.0 \mathrm{~Hz}$, $\left.6 \mathrm{H}, \mathrm{CH}_{2}(9)\right) ;-0.04$ (s, 9H, $\left.\mathrm{CH}_{3}(14)\right)$

${ }^{13} \mathrm{C}$ NMR: $\quad\left(126 \mathrm{MHz}, \mathrm{CDCl}_{3}\right)$

$162.45\left(\mathrm{C}\left(2^{\prime}\right)\right)$; $155.76(\mathrm{C}(11)) ; 154.63\left(\mathrm{C}\left(4^{\prime}\right)\right) ; 143.66\left(\mathrm{C}\left(1^{\prime}\right)\right) ; 137.33\left(\mathrm{C}\left(10^{\prime}\right.\right.$ or $\left.\left.14^{\prime}\right)\right) ; 136.78\left(\mathrm{C}\left(10^{\prime}\right.\right.$ or $\left.\left.14^{\prime}\right)\right) ; 128.85\left(\mathrm{C}\left(15^{\prime}, 16^{\prime}, 7^{\prime}, 11^{\prime}, 12^{\prime}, 13^{\prime}\right)\right) ; 128.58\left(\mathrm{C}\left(15^{\prime}\right.\right.$, 16', 17', 11', 12', 13')); 128.33 (C(15', 16', 17', 11', 12', 13')); 127.84 (C(15', 16', 17', 11', 12', 13')); 127.67 (C(15', 16', 17', 11', 12', 13')); 127.02 (C(15', 16', 17', 11', 12', 13')); $117.17\left(\mathrm{C}\left(6^{\prime}\right)\right) ; 109.66(\mathrm{C}(1)) ; 100.43\left(\mathrm{C}\left(3^{\prime}\right)\right) ; 98.20\left(\mathrm{C}\left(5^{\prime}\right)\right) ; 78.10$ (C(4)); $77.50(\mathrm{C}(2)) ; 74.24(\mathrm{C}(5)) ; 73.76(\mathrm{C}(3)) ; 70.55\left(\mathrm{C}\left(8^{\prime}\right.\right.$ or $\left.\left.9^{\prime}\right)\right) ; 70.27\left(\mathrm{C}\left(8^{\prime}\right.\right.$ or 9')); $68.97(\mathrm{C}(6)) ; 67.25\left(\mathrm{C}\left(7^{\prime}\right)\right) ; 66.21(\mathrm{C}(12)) ; 27.84(\mathrm{C}(8 \mathrm{a}$ or $8 \mathrm{~b})) ; 27.31(\mathrm{C}(8 \mathrm{a}$ or $8 \mathrm{~b})$ ); $23.09(\mathrm{C}(7 \mathrm{a}$ or $7 \mathrm{~b})) ; 20.20(\mathrm{C}(7 \mathrm{a}$ or $7 \mathrm{~b})) ; 17.48(\mathrm{C}(13)) ; 7.15(\mathrm{C}(10))$; $5.29(\mathrm{C}(9)) ;-1.36(\mathrm{C}(14))$

IR: (film)

2952 (s); 2935 (s); 2861 (s); 1750 (s); 1611 (s); 1445 (w); 1456 (w); 1385 (w); 
1253 (s); 1263 (s); 1163 (m); 1094 (m); 1070 (m); 1023 (m); 836 (s); 740 (m)

MS: (ESI)

$879\left(\mathrm{M}^{+}(80)\right) ; 873$ (15); 854 (2); 852 (24); 851 (100)

Opt. rot. : $\quad[\alpha]_{D}^{24}-35.30(\mathrm{EtOH}, \mathrm{c}=0.90)$

TLC: $\quad R_{f} 0.22\left(10: 1\right.$ hexanes/EtOAc) $\left[\mathrm{SiO}_{2}, \mathrm{CAM}\right]$

Analysis: $\quad \mathrm{C}_{47} \mathrm{H}_{70} \mathrm{O}_{10} \mathrm{Si}_{3}(879.31)$

Calcd: $\quad$ C, $64.20 \% ; \quad H, 8.02 \%$

Found: $\quad$ C, $63.85 \% ; \quad H, 8.04 \%$

Preparation of $\left(1 R, 4^{\prime} \mathrm{a} R, 7^{\prime} R, 8^{\prime} R, 8^{\prime} \mathrm{a} R\right)-\mathrm{Spiro}$ [isobenzofuran-1(3H),6'(4'H)-pyrano[3,2d] [1,3,2]dioxasilin]-7'-ol, $\quad 2$ ',2'-bis(1,1-dimethylethyl)-4'a,7',8',8'a-tetrahydro-5, 7,bis(hydroxy)-8'- triethylsilyl-9'-(2-trimethylsilylethoxycarbonyl) (18) [CSR-VIII-79]

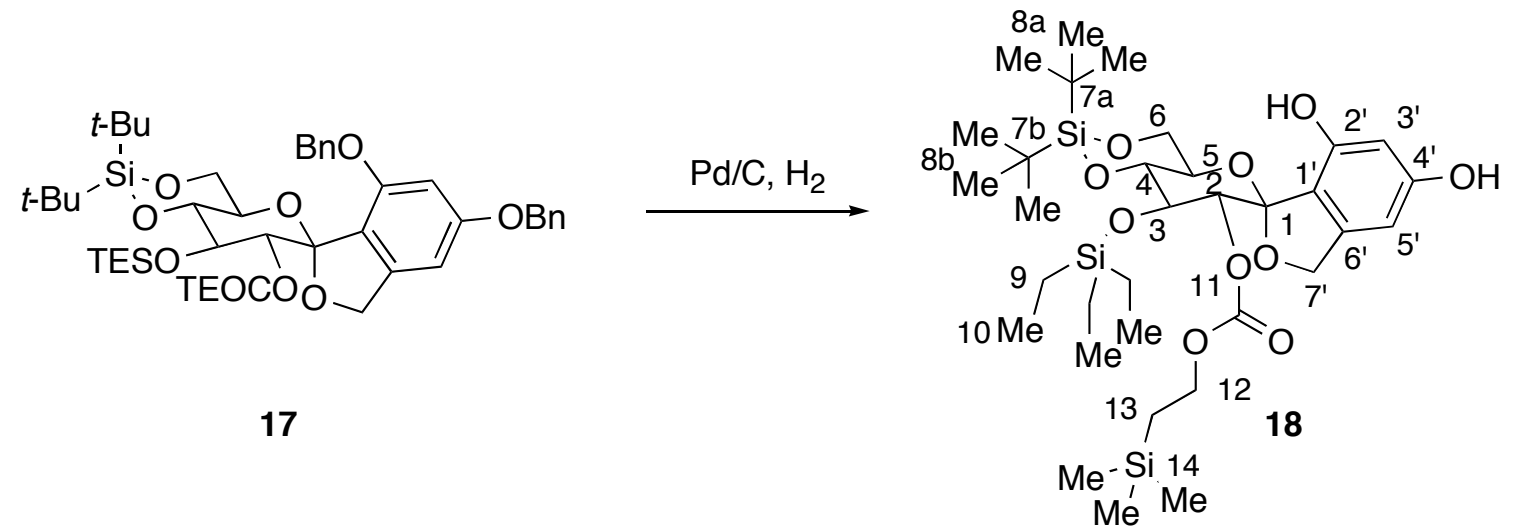

To a 100-mL, one-necked, round-bottom flask, equipped with a magnetic stir-bar was added 17 (0.731 g, $0.831 \mathrm{mmol}, 1.0$ equiv) followed by THF $(50 \mathrm{~mL})$. Then $\mathrm{NaHCO}_{3}(0.451 \mathrm{~g}$, $5.37 \mathrm{mmol}, 6.5$ equiv) was added along with $5 \%$ palladium on carbon (451 $\mathrm{mg}, 0.211 \mathrm{mmol}$, 0.25 equiv). The flask was attached to hydrogen manifold and the flask was purged with hydrogen (3x) and stirred at room temperature under 1 atm of $\mathrm{H}_{2}$ for $5 \mathrm{~h}$. The flask and manifold were flushed with nitrogen and the contents were filtered through Celite $(1 \mathrm{~g})$ and the filter pad was washed with $\mathrm{Et}_{2} \mathrm{O}(30 \mathrm{~mL})$. The colorless filtrate was concentrated to a foamy solid using rotary evaporation. The solid was further dried overnight at $30{ }^{\circ} \mathrm{C}(6 \mathrm{~h})$ under high vacuum $(0.06 \mathrm{mmHg})$ to afford $0.581 \mathrm{~g}$ (quantitative) of $\mathbf{1 8}$ as a powdery, white solid.

\section{Data for 18:}

mp: $\quad 106-108^{\circ} \mathrm{C}(\mathrm{THF})$ 
${ }^{1}$ H NMR: $\quad\left(500 \mathrm{MHz}, \mathrm{CDCl}_{3}\right)$

$6.35\left(\mathrm{~d}, J=2.0 \mathrm{~Hz}, 1 \mathrm{H}, \mathrm{CH}\left(3^{\prime}\right)\right) ; 6.22\left(\mathrm{~d}, J=2.0 \mathrm{~Hz}, 1 \mathrm{H}, \mathrm{CH}\left(5^{\prime}\right)\right) ; 6.20(\mathrm{~s}, 1 \mathrm{H}$, $\operatorname{br}(\mathrm{OH})$ ); 6.09 (s, 1H, br(OH)); $5.26(\mathrm{~d}, J=9.0 \mathrm{~Hz}, 1 \mathrm{H}, \mathrm{CH}(2)) ; 5.08$ (d, $J=11.8$ $\left.\mathrm{Hz}, 1 \mathrm{H}, \mathrm{CH}\left(7 \mathrm{a}^{\prime}\right)\right) ; 5.05$ (d, $\left.J=11.8 \mathrm{~Hz}, 1 \mathrm{H}, \mathrm{CH}\left(7 \mathrm{~b}^{\prime}\right)\right) ; 4.15$ (dd, $J=10.0,4.6 \mathrm{~Hz}$, 1H, $\mathrm{CH}(6 \mathrm{e})$ ); 4.11 (t, $J=8.8 \mathrm{~Hz}, 1 \mathrm{H}, \mathrm{CH}(3)) ; 4.01$ (t, $\left.J=8.5 \mathrm{~Hz}, 2 \mathrm{H}, \mathrm{CH}_{2}(12)\right)$; 4.07-4.00 (m, 1H, CH(5)); 3.95 (t, $J=8.8 \mathrm{~Hz}, 1 \mathrm{H}, \mathrm{CH}(4)) ; 3.90$ (t, $J=10.0 \mathrm{~Hz}$, $1 \mathrm{H}, \mathrm{CH}(6 \mathrm{a})) ; 1.08$ (s, 9H, $\mathrm{C}\left(\mathrm{CH}_{3}\right)_{3}(8 \mathrm{a}$ or $\left.8 \mathrm{~b})\right) ; 1.04$ (s, 9H, $\mathrm{C}\left(\mathrm{CH}_{3}\right)_{3}(8 \mathrm{a}$ or $8 \mathrm{~b})$ ); 0.97 (t, $\left.J=8.1 \mathrm{~Hz}, 9 \mathrm{H}, \mathrm{CH}_{3}(10)\right) ; 0.84$ (dt, $J=8.5,2.0 \mathrm{~Hz}, 2 \mathrm{H}, \mathrm{CH}_{2}(13)$ ); 0.68 (dq, $\left.J=8.1,2.0 \mathrm{~Hz}, 2 \mathrm{H}, \mathrm{CH}_{2}(9)\right) ;-0.04$ (s, 9H, $\left.\mathrm{Si}\left(\mathrm{CH}_{3}\right)_{3}(14)\right)$

${ }^{13}$ C NMR: $\quad\left(126 \mathrm{MHz}, \mathrm{CDCl}_{3}\right)$

$159.49\left(\mathrm{C}\left(2^{\prime}\right)\right) ; 155.42(\mathrm{C}(11)) ; 151.89\left(\mathrm{C}\left(4^{\prime}\right)\right) ; 143.57\left(\mathrm{C}\left(1^{\prime}\right)\right) ; 115.02\left(\mathrm{C}\left(6^{\prime}\right)\right)$; $108.80(\mathrm{C}(1)) ; 103.60\left(\mathrm{C}\left(3^{\prime}\right)\right) ; 100.51\left(\mathrm{C}\left(5^{\prime}\right)\right) ; 78.31(\mathrm{C}(4)) ; 77.78(\mathrm{C}(2)) ; 73.98$ (C(5)); $73.61(\mathrm{C}(3)) ; 68.85(\mathrm{C}(6))$; $66.95\left(\mathrm{C}\left(7^{\prime}\right)\right)$; $66.75(\mathrm{C}(12)) ; 27.60$ (C(8a or $8 \mathrm{~b})) ; 27.06(\mathrm{C}(8 \mathrm{a}$ or $8 \mathrm{~b})) ; 22.74(\mathrm{C}(7 \mathrm{a}$ or $7 \mathrm{~b})) ; 19.99$ (C(7a or 7b)); $17.77(\mathrm{C}(13))$; $6.87(\mathrm{C}(10)) ; 5.05(\mathrm{C}(9)) ;-1.68(\mathrm{C}(14))$

IR: (film)

3413 (m); 2954 (s); 2936 (s); 2878 (s); 2913 (m); 2861 (m); 1727 (m); 1612 (m); 1473 (m); 1388 (m); 1341 (w); 1278 (s); 1252 (s); 1167 (m); 1094 (m); 1066 (s) $979(\mathrm{~m})$

MS: (ESI)

$699\left(\mathrm{M}^{+}(55)\right) ; 609(5) ; 402(3) ; 383(5) ; 372$ (2)

Opt. rot. : $\quad[\alpha]_{\mathrm{D}}^{24}-23.80(\mathrm{EtOH}, \mathrm{c}=0.50)$

TLC: $\quad R_{f} 0.20\left(10: 1\right.$ hexanes/EtOAc) $\left[\mathrm{SiO}_{2}, \mathrm{CAM}\right]$

Analysis: $\quad \mathrm{C}_{33} \mathrm{H}_{58} \mathrm{O}_{10} \mathrm{Si}_{3}(699.06)$

Calcd: $\quad$ C, $56.70 \% ; \quad$ H, $8.36 \%$

Found: $\quad$ C, $56.50 \%$; $\quad \mathrm{H}, 8.11 \%$ 
Preparation of $\left(1 R, 4^{\prime} \mathrm{a} R, 7^{\prime} R, 8^{\prime} R, 8^{\prime} \mathrm{a} R\right)$-Spiro[isobenzofuran-1(3H),6'(4'H)-pyrano[3,2d] [1,3,2] dioxasilin]-7'-ol, 2',2'-bis(1,1-dimethylethyl)-4'a,7', 8',8'a-tetrahydro-5,7,- $\quad$ bis(2trimethylsilylethoxycarbonyl)-8'-triethylsilyl-9'-(2-trimethylsilylethoxycarbonyl) [CSR-VII-62]

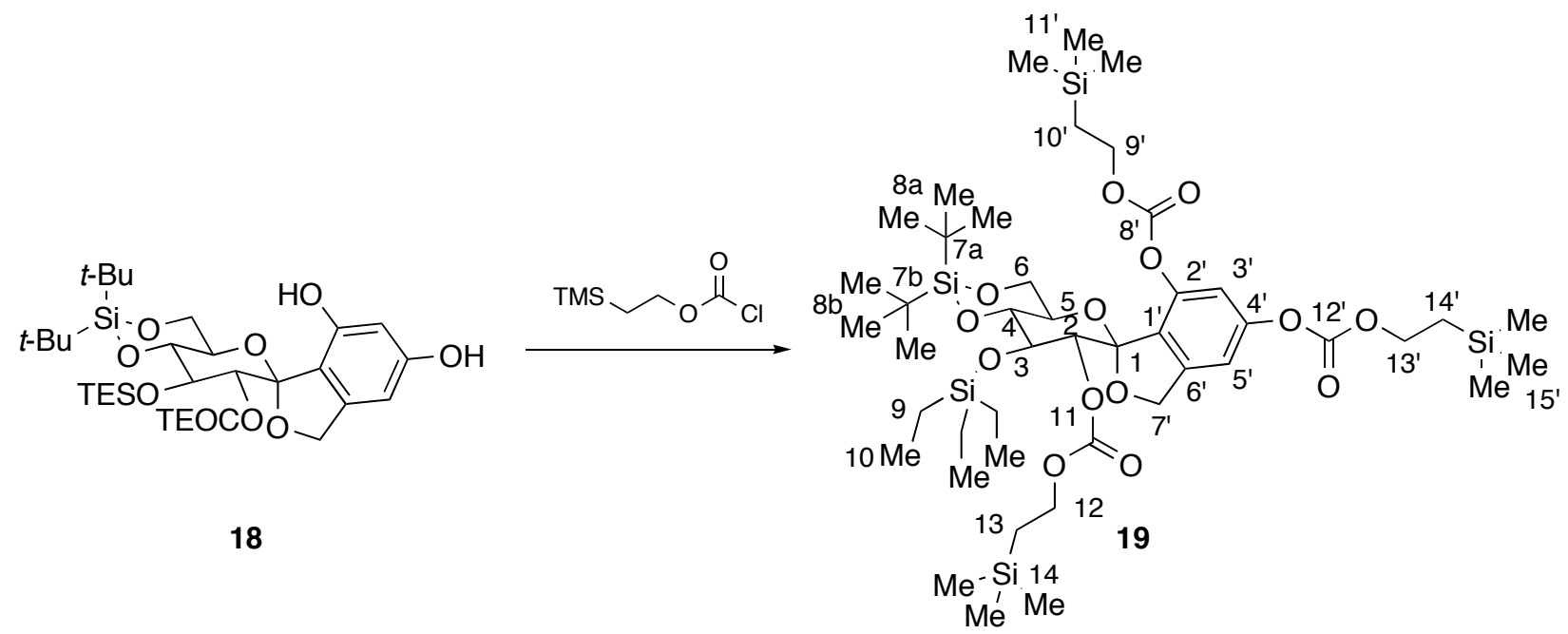

To a 25-mL Schlenk flask, equipped with a magnetic stir-bar and an argon inlet with a septum was added 18 (0.273 g, $0.391 \mathrm{mmol}, 1.0$ equiv) and the flask was charged with argon. Then $\mathrm{CH}_{2} \mathrm{Cl}_{2}(5.0 \mathrm{~mL})$ was added along with $i$ - $\operatorname{Pr}_{2} \mathrm{NEt}(681 \mu \mathrm{L}, 3.91 \mathrm{mmol}, 10$ equiv). Trimethylsilylethoxy chlorocarbonate $(0.354 \mathrm{~g}, 1.96 \mathrm{mmol}, 5.0$ equiv) was added drop wise to the colorless solution, which turned light-yellow after the addition. The solution was stirred at room temperature $10 \mathrm{~h}$. Then $\mathrm{H}_{2} \mathrm{O}(30 \mathrm{~mL})$ was added and the contents were transferred to a 125-mL separatory funnel and the aqueous was extracted. The aqueous extracted was extracted with EtOAc $(3 \times 15 \mathrm{~mL})$. The combined organic extracts were washed with brine $(1 \times 15 \mathrm{~mL})$, dried $\left(\mathrm{Na}_{2} \mathrm{SO}_{4}\right)$, filtered and concentrated to a light-yellow wax. The crude product was purified using column chromatography $\left(\left(\mathrm{SiO}_{2}, 30 \times 80 \mathrm{~mm}\right)\right.$, hexanes/EtOAc, 15/1 to 10/1). The purified product was isolated as a viscous oil that was dried at $50{ }^{\circ} \mathrm{C}$ for $18 \mathrm{~h}$ to afford $0.371 \mathrm{~g}(96 \%)$ of 19 as a colorless glassy solid.

Data for 19:

mp: $\quad 40-42{ }^{\circ} \mathrm{C}(10 / 1$ hexanes/EtOAc $)$

${ }^{1} \mathrm{H} \mathrm{NMR}: \quad\left(500 \mathrm{MHz}, \mathrm{CDCl}_{3}\right)$

$7.10\left(\mathrm{~d}, J=2.0 \mathrm{~Hz}, 1 \mathrm{H}, \mathrm{CH}\left(3^{\prime}\right.\right.$ or $\left.\left.5^{\prime}\right)\right) ; 6.95\left(\mathrm{~d}, J=2.0 \mathrm{~Hz}, 1 \mathrm{H}, \mathrm{CH}\left(3^{\prime}\right.\right.$ or $\left.\left.5^{\prime}\right)\right) ; 5.35$

$(\mathrm{d}, J=9.5 \mathrm{~Hz}, 1 \mathrm{H}, \mathrm{CH}(2)) ; 5.16\left(\mathrm{~s}, 2 \mathrm{H}, \mathrm{CH}_{2}\left(7^{\prime}\right)\right) ; 4.274 .38\left(\mathrm{~m}, 4 \mathrm{H}, \mathrm{CH}_{2}\left(9^{\prime}\right)\right.$ and $13 ')) ; 4.09(\mathrm{dd}, J=9.8,4.8 \mathrm{~Hz}, 1 \mathrm{H}, \mathrm{CH}(6 \mathrm{e}))$; 4.07 (t, $J=9.2 \mathrm{~Hz}, 1 \mathrm{H}, \mathrm{CH}(3))$; 
3.94-4.03 (m, 3H, $\mathrm{CH}_{2}(12)$ and $\left.\mathrm{CH}(5)\right) ; 3.88(\mathrm{t}, J=9.2 \mathrm{~Hz}, 1 \mathrm{H}, \mathrm{CH}(4)) ; 3.77$ (t, $J$ $=9.8 \mathrm{~Hz}, 1 \mathrm{H}, \mathrm{CH}(6 \mathrm{a})) ; 1.09-1.15\left(\mathrm{~m}, 4 \mathrm{H}, \mathrm{CH}_{2}\left(10^{\prime}\right.\right.$ and $\left.\left.14{ }^{\prime}\right)\right) ; 1.08(\mathrm{~s}, 9 \mathrm{H}$, $\mathrm{C}\left(\mathrm{CH}_{3}\right)_{3}(8 \mathrm{a}$ or $\left.8 \mathrm{~b})\right) ; 1.03\left(\mathrm{~s}, 9 \mathrm{H}, \mathrm{C}\left(\mathrm{CH}_{3}\right)_{3}(8 \mathrm{a}\right.$ or $\left.8 \mathrm{~b})\right) ; 0.97(\mathrm{t}, J=8.1 \mathrm{~Hz}, 9 \mathrm{H}$, $\mathrm{CH}_{3}(10)$ ); 0.81-0.86 (m, 2H, $\left.\mathrm{CH}_{2}(13)\right) ; 0.66$ (q, $J=8.1 \mathrm{~Hz}, 6 \mathrm{H}, \mathrm{CH}_{2}(9)$ ); 0.08 (s, $9 \mathrm{H}, \mathrm{CH}\left(11^{\prime}\right.$ or $\left.\left.15^{\prime}\right)\right) ; 0.07\left(\mathrm{~s}, 9 \mathrm{H}, \mathrm{CH}\left(11^{\prime}\right.\right.$ or $\left.\left.15^{\prime}\right)\right) ;-0.05(\mathrm{~s}, 9 \mathrm{H}, \mathrm{CH}(14))$

${ }^{13} \mathrm{C} \mathrm{NMR}: \quad\left(126 \mathrm{MHz}, \mathrm{CDCl}_{3}\right)$

$154.23(\mathrm{C}(11)) ; 153.06\left(\mathrm{C}\left(2^{\prime}\right)\right) ; 152.83\left(\mathrm{C}\left(8^{\prime}\right.\right.$ or $\left.\left.12^{\prime}\right)\right) ; 152.56\left(\mathrm{C}\left(8^{\prime}\right.\right.$ or $\left.\left.12^{\prime}\right)\right) ; 146.82$ $\left(\mathrm{C}\left(4^{\prime}\right)\right) ; 143.16\left(\mathrm{C}\left(1^{\prime}\right)\right) ; 124.93\left(\mathrm{C}\left(6^{\prime}\right)\right) ; 115.25\left(\mathrm{C}\left(3^{\prime}\right)\right) ; 111.35\left(\mathrm{C}\left(5^{\prime}\right)\right) ; 108.83$ $(\mathrm{C}(1)) ; 77.72 \quad(\mathrm{C}(4)) ; 76.69$ (C(2)); 73.69 (C(5)); 73.16 (C(3)); 68.89 (C(6)); $67.64\left(\mathrm{C}\left(9^{\prime}\right.\right.$ or $\left.\left.13^{\prime}\right)\right) ; 67.57\left(\mathrm{C}\left(9^{\prime}\right.\right.$ or $\left.\left.13^{\prime}\right)\right) ; 66.74\left(\mathrm{C}\left(7^{\prime}\right)\right) ; 66.36(\mathrm{C}(12)) ; 27.55(\mathrm{C}(8 \mathrm{a}$ or $8 \mathrm{~b})) ; 27.02(\mathrm{C}(8 \mathrm{a}$ or $8 \mathrm{~b})) ; 22.76(\mathrm{C}(7 \mathrm{a}$ or $7 \mathrm{~b})) ; 19.95(\mathrm{C}(7 \mathrm{a}$ or $7 \mathrm{~b})) ; 17.51$ $\left(\mathrm{C}\left(10^{\prime}\right.\right.$ or $\left.\left.14^{\prime}\right)\right)$; $17.47\left(\mathrm{C}\left(10^{\prime}\right.\right.$ or $\left.\left.14^{\prime}\right)\right) ; 17.17(\mathrm{C}(13))$; $6.87(\mathrm{C}(10))$; $4.99(\mathrm{C}(9))$; $1.53\left(\mathrm{C}\left(11^{\prime}\right.\right.$ or $\left.\left.15^{\prime}\right)\right) ;-1.58\left(\mathrm{C}\left(11^{\prime}\right.\right.$ or $\left.\left.15^{\prime}\right)\right) ;-1.70(\mathrm{C}(14))$

IR: (film)

2954 (s); 2878 (m); 1766 (s); $1694(\mathrm{w}) ; 1628(\mathrm{w}) ; 1473(\mathrm{w}) ; 1383(\mathrm{w}) ; 1228$ (s); 1175 (s); 1066 (s); 1032 (m); 971 (m); 936 (m); 859 (s)

MS: (ESI)

$1009\left(\mathrm{M}^{+}+\mathrm{Na}(100)\right) ; 904$ (40); 903 (50); 485 (100); 469 (20)

Opt. rot. : $\quad[\alpha]_{\mathrm{D}}^{24}-19.07(\mathrm{EtOH}, \mathrm{c}=0.30)$

TLC: $\quad R_{f} 0.20\left(15: 1\right.$ hexanes/EtOAc) $\left[\mathrm{SiO}_{2}, \mathrm{CAM}\right]$

HRMS: $\quad \mathrm{C}_{45} \mathrm{H}_{82} \mathrm{O}_{14} \mathrm{Si}_{5} \mathrm{Na}(1009.44)$

Calcd: $\quad 1009.4449$

Found: $\quad 1009.4443$

Analysis: $\quad \mathrm{C}_{45} \mathrm{H}_{82} \mathrm{O}_{14} \mathrm{Si}_{5}(987.55)$

Calcd: $\quad$ C, $54.73 \% ; \quad H, 8.37 \%$

Found: $\quad$ C, $54.61 \% ; \quad H, 8.58 \%$ 


\section{Preparation of $\left(1 R, 4^{\prime} \mathrm{a} R, 7^{\prime} R, 8^{\prime} R, 8^{\prime} \mathrm{a} R\right)$-Spiro[isobenzofuran-1(3H), 6'(4'H)-pyrano[3,2- d] [1,3,2]dioxasilin]-7'-ol, 2',2'-bis(1,1-dimethylethyl)-4'a,7',8', 8'a-tetrahydro-5,7,- bis(2- trimethylsilylethoxycarbonyl)-9'-(2-trimethylsilylethoxycarbonyl) \\ (20) [CSR-IX-1]}

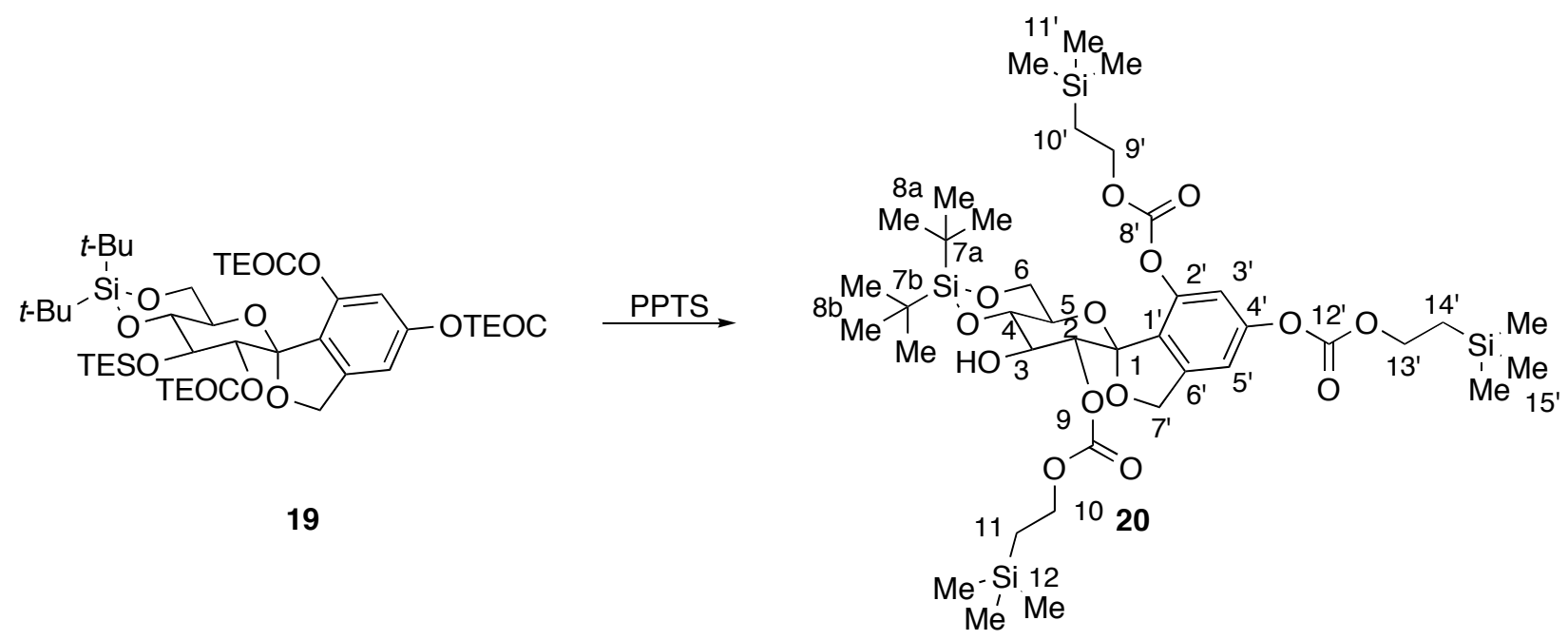

To a 35-mL, one-necked, round-bottom flask, equipped with a magnetic stir-bar and an argon inlet with a septum was added $19(0.622 \mathrm{mg}, 0.639 \mathrm{mg}, 1.0$ equiv) followed by absolute EtOH $(17 \mathrm{~mL})$. Then pyridium-p-toluenesulfonate $(1.1 \mathrm{~g}, 1.26 \mathrm{mmol}, 2.0$ equiv) was added and the contents were stirred at room temperature for $24 \mathrm{~h}$. The colorless solution was transferred to a $250-\mathrm{mL}$ separatory funnel and $\mathrm{H}_{2} \mathrm{O}(30 \mathrm{~mL})$ was added along with EtOAc $(100 \mathrm{~mL})$. The aqueous extract was extracted with EtOAc $(5 \times 25 \mathrm{~mL})$. The combined organic extracts were washed with brine $(1 \times 20 \mathrm{~mL})$, dried $\left(\mathrm{Na}_{2} \mathrm{SO}_{4}\right)$, filtered and concentrated to a viscous colorless oil. The crude product was purified using column chromatography (( $\left.\mathrm{SiO}_{2}, 40 \times 150 \mathrm{~mm}\right)$, hexanes/EtOAc, 7/1). The purified product was isolated as a viscous oil that was dried at $70{ }^{\circ} \mathrm{C}$ for $8 \mathrm{~h}$ to afford $0.497 \mathrm{~g}(93 \%)$ of $\mathbf{2 0}$ as a colorless, glassy solid.

Data for 20:

mp: $\quad 51-54{ }^{\circ} \mathrm{C}(7 / 1$ hexanes/EtOAc $)$

${ }^{1} \mathrm{H} \mathrm{NMR}: \quad\left(500 \mathrm{MHz}, \mathrm{CDCl}_{3}\right)$

$7.11\left(\mathrm{~d}, J=1.7 \mathrm{~Hz}, 1 \mathrm{H}, \mathrm{CH}\left(3^{\prime}\right.\right.$ or $\left.\left.5^{\prime}\right)\right) ; 6.97\left(\mathrm{~d}, J=1.7 \mathrm{~Hz}, 1 \mathrm{H}, \mathrm{CH}\left(3^{\prime}\right.\right.$ or 5')); 5.48 $(\mathrm{d}, J=10.0 \mathrm{~Hz}, 1 \mathrm{H}, \mathrm{CH}(2)) ; 5.20$ (d, $\left.J=5.2 \mathrm{~Hz}, 1 \mathrm{H}, \mathrm{CH}\left(7^{\prime} \mathrm{a}\right)\right) ; 5.17$ (d, $J=5.2$ $\left.\mathrm{Hz}, 1 \mathrm{H}, \mathrm{CH}\left(7^{\prime} \mathrm{b}\right)\right)$; 4.28-4.38 (m, 4H, $\mathrm{CH}_{2}\left(9^{\prime}\right.$ and 13')); 4.07-4.13 (m, 2H, CH(6e and 3)); 3.99-4.08 (m, 4H, $\mathrm{CH}_{2}(10)$ and $\mathrm{CH}(6 \mathrm{a})$ and $\left.\mathrm{CH}(4)\right) ; 2.63(\mathrm{~d}, J=2.4 \mathrm{~Hz}$,

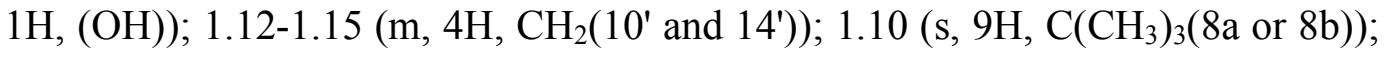


$1.02\left(\mathrm{~s}, 9 \mathrm{H}, \mathrm{C}\left(\mathrm{CH}_{3}\right)_{3}(8 \mathrm{a}\right.$ or $\left.8 \mathrm{~b})\right) ; 0.97$ (t, $\left.J=8.8 \mathrm{~Hz}, 2 \mathrm{H}, \mathrm{CH}_{2}(11)\right) ; 0.07$ (s, 9H, $\mathrm{CH}_{3}\left(11^{\prime}\right.$ or $\left.\left.15^{\prime}\right)\right) ; 0.07$ (s, $9 \mathrm{H}, \mathrm{CH}_{3}\left(11^{\prime}\right.$ or $\left.\left.15^{\prime}\right)\right)$; -0.05 (s, $\left.9 \mathrm{H}, \mathrm{CH}_{3}(12)\right)$

${ }^{13} \mathrm{C} \mathrm{NMR}: \quad\left(126 \mathrm{MHz}, \mathrm{CDCl}_{3}\right)$

$154.51(\mathrm{C}(9)) ; 153.16\left(\mathrm{C}\left(2^{\prime}\right)\right) ; 152.86\left(\mathrm{C}\left(8^{\prime}\right.\right.$ or $\left.\left.12^{\prime}\right)\right) ; 152.52\left(\mathrm{C}\left(8^{\prime}\right.\right.$ or $\left.\left.12^{\prime}\right)\right) ; 146.69$ $\left(\mathrm{C}\left(4^{\prime}\right)\right) ; 143.26\left(\mathrm{C}\left(1^{\prime}\right)\right) ; 124.75\left(\mathrm{C}\left(6^{\prime}\right)\right) ; 115.31$ (C(3')); 111.52 (C(5')); 108.59 (C(1)); $77.36(\mathrm{C}(4)) ; 75.94(\mathrm{C}(2)) ; 72.98(\mathrm{C}(5))$; 68.44(C(3)); 67.75 (C(9' or 13')); $67.72\left(\mathrm{C}\left(9^{\prime}\right.\right.$ or $\left.\left.13^{\prime}\right)\right)$; $66.90(\mathrm{C}(6))$; $66.77\left(\mathrm{C}\left(7^{\prime}\right)\right)$; $66.54(\mathrm{C}(10)) ; 27.44$ (C(8a or 8b)); 27.01 (C(8a or 8b)); 22.71 (C(7a or 7b)); 19.98 (C(7a or 7b)); $17.54\left(\mathrm{C}\left(10^{\prime}\right.\right.$ or $\left.\left.14^{\prime}\right)\right)$; $17.50\left(\mathrm{C}\left(10^{\prime}\right.\right.$ or $\left.\left.14^{\prime}\right)\right)$; $17.12(\mathrm{C}(11))$; $-1.53\left(\mathrm{C}\left(11^{\prime}\right.\right.$ or $\left.\left.15^{\prime}\right)\right)$; $-1.57\left(\mathrm{C}\left(11^{\prime}\right.\right.$ or $\left.\left.15^{\prime}\right)\right) ;-1.64(\mathrm{C}(12))$

IR: (film)

$2954(\mathrm{~s}) ; 2878(\mathrm{~m}) ; 1766(\mathrm{~s}) ; 1694(\mathrm{w}) ; 1628(\mathrm{w}) ; 1473(\mathrm{w}) ; 1383(\mathrm{w}) ; 1228(\mathrm{~s})$ 1175 (s); 1066 (s); 1032 (m); $971(\mathrm{~m}) ; 936$ (m); 859 (s)

MS: (ESI)

$1009\left(\mathrm{M}^{+}+\mathrm{Na}(100)\right) ; 904$ (40); 903 (50); 485 (100); 469 (20)

Opt. rot. : $\quad[\alpha]_{\mathrm{D}}^{24}-19.07(\mathrm{EtOH}, \mathrm{c}=0.30)$

TLC: $\quad R_{f} 0.20\left(15: 1\right.$ hexanes/EtOAc) $\left[\mathrm{SiO}_{2}, \mathrm{CAM}\right]$

Analysis: $\quad \mathrm{C}_{39} \mathrm{H}_{68} \mathrm{O}_{14} \mathrm{Si}_{4}(873.29)$

Calcd: $\quad$ C, $53.64 \% ; \quad H, 7.85 \%$

Found: $\quad$ C, $53.68 \% ; \quad H, 8.05 \%$

\section{Preparation of Acid 2}

Preparation of $(S)$ - 2,6-Dimethyl-, 6-octene-1-ol, 4'-methylbenzenesulfonate (22) ${ }^{16}$ [CSRVII-63]
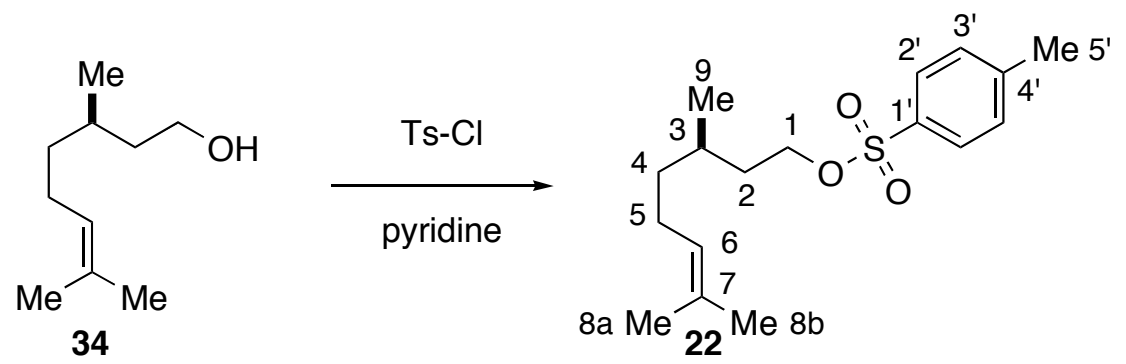

A 50-mL, two-necked, round-bottom flask, containing a magnetic stir-bar, glass stopcock, and nitrogen inlet was charged with 34 (6.0 g, 38.9 mmol, 1.0 equiv), pyridine (4.72 $\mathrm{mL}, 58.4 \mathrm{mmol}, 1.5 \mathrm{equiv})$. To this solution finely ground $p$-toluenesulfonyl chloride $(8.9 \mathrm{~g}$, 
$46.7 \mathrm{mmol}, 1.2$ equiv) was added in three portions of approximately $3 \mathrm{~g}$ each. The internal temperature rose to $52{ }^{\circ} \mathrm{C}$ and a white suspension formed upon cooling to room temperature. The thick, white suspension was stirred at room temperature for $10 \mathrm{~h}$. The reaction mixture was diluted with EtOAc $(20 \mathrm{~mL})$ and $2 \mathrm{~N} \mathrm{HCl}(10 \mathrm{~mL})$, and stirred until the biphasic contents were homogenous ( $\sim 3 \mathrm{~min}$.). The contents were transferred to a $250-\mathrm{mL}$ separatory funnel and the aqueous layer was extracted. The aqueous phase was back-extracted with EtOAc $(2 \times 15 \mathrm{~mL})$, the organic layers combined, washed with water $(3 \times 10 \mathrm{~mL})$ and brine $(20 \mathrm{~mL})$. The organic layer was dried $\left(\mathrm{Na}_{2} \mathrm{SO}_{4}\right)$, filtered and concentrated under reduced pressure using rotary evaporation to afford a colorless oil. The crude material was purified by column chromatography $\left(\left(\mathrm{SiO}_{2}, 60 \times 120 \mathrm{~mm}\right)\right.$, hexanes/EtOAc, $\left.7 / 1\right)$ to afford $10.7 \mathrm{~g}(89 \%)$ of $\mathbf{2 2}$ as a colorless oil.

Data for 22:

${ }^{1}$ H NMR: $\quad\left(500 \mathrm{MHz}, \mathrm{CDCl}_{3}\right)$

$7.79\left(\mathrm{~d}, J=8.3 \mathrm{~Hz}, 2 \mathrm{H}, \mathrm{CH}\left(2^{\prime}\right)\right) ; 7.34\left(\mathrm{~d}, J=8.3 \mathrm{~Hz}, 2 \mathrm{H}, \mathrm{CH}\left(3^{\prime}\right)\right) ; 5.02(\mathrm{t}, J=7.3$ $\mathrm{Hz}, 1 \mathrm{H}, \mathrm{CH}(6))$; 4.02-4.10 (m, $\left.2 \mathrm{H}, \mathrm{CH}_{2}(1)\right) ; 2.45$ (s, $3 \mathrm{H}, \mathrm{CH}_{3}\left(5^{\prime}\right)$ ); 1.83-1.98 (m, $\left.2 \mathrm{H}, \mathrm{CH}_{2}(5)\right) ; 1.71-1.64\left(\mathrm{~m}, 2 \mathrm{H}, \mathrm{CH}_{2}(3)\right) ; 1.67$ (s, 3H, $\mathrm{CH}_{3}(8 \mathrm{a}$ or $8 \mathrm{~b})$ ); 1.57 (s, $3 \mathrm{H}, \mathrm{CH}_{3}(8 \mathrm{a}$ or $\left.8 \mathrm{~b})\right)$; $1.56-1.49(\mathrm{~m}, 1 \mathrm{H}, \mathrm{CH}(2 \mathrm{a})) ; 1.39-1.46(\mathrm{~m}, 1 \mathrm{H}, \mathrm{CH}(2 \mathrm{~b})$ ); 1.21-1.27 (m, 1H, CH(4a)); 1.06-1.13 (m, 1H, CH(4b)); 0.82 (d, $J=6.6 \mathrm{~Hz}, 3 \mathrm{H}$, $\left.\mathrm{CH}_{3}(9)\right)$

${ }^{13}$ C NMR: $\quad\left(126 \mathrm{MHz}, \mathrm{CDCl}_{3}\right)$

$144.87\left(\mathrm{C}\left(4^{\prime}\right)\right) ; 133.45(\mathrm{C}(7)) ; 131.73\left(\mathrm{C}\left(1^{\prime}\right)\right) ; 130.04\left(\mathrm{C}\left(2^{\prime}\right)\right) ; 128.13\left(\mathrm{C}\left(3^{\prime}\right)\right)$; $124.55(\mathrm{C}(6)) ; 69.29$ (C(1)); $36.94(\mathrm{C}(4)) ; 35.89$ (C(2)); 29.08 (C(3)); 25.94 (C(8a or $8 \mathrm{~b})) ; 25.49(\mathrm{C}(5)) ; 21.88\left(\mathrm{C}\left(5^{\prime}\right)\right) ; 19.27(\mathrm{C}(9)) ; 17.87(\mathrm{C}(8 \mathrm{a}$ or $8 \mathrm{~b}))$

IR: (neat)

2963 (s); 2925 (s); 2873 (s); 2856 (s); 2730 (w); 2345 (w); 1920 (w); 1805 (w); 1654 (w); 1598 (m); 1495 (w); 1454 (m); 1361 (s); 1307 (w); 1291 (w); 1189 (s); 1177 (s); 1038 (w); 1020 (w); 945 (s); 889 (s); 815 (s); 764 (m); 665 (s)

MS: $\quad(\mathrm{EI}, 70 \mathrm{eV})$

$310\left(\mathrm{M}^{+}(3)\right) ; 173$ (11); 155 (34); 138 (61); 123 (59); 109 (32); 95 (54); 92 (14); 91(100); 89 (20); 81 (49); 79 (12); 69 (73); 68 (33); 67 (40); 65 (70); 63 (17); 55 (37); 53 (18)

Opt. rot. : $\quad[\alpha]_{\mathrm{D}}^{24} 2.68(\mathrm{EtOH}, \mathrm{c}=1.00)$ 
TLC: $\quad R_{f} 0.30\left(10: 1\right.$ hexanes/EtOAc) $\left[\mathrm{SiO}_{2}, \mathrm{UV}\right]$

HRMS: for $\mathrm{C}_{17} \mathrm{H}_{26} \mathrm{O}_{3} \mathrm{~S}$

Calcd: $\quad 310.1603$

Found: $\quad 310.1605$

\section{Preparation of (S)- 2,6-Dimethyl-2-octene (23) ${ }^{7}$ [CSR-VII-88]}

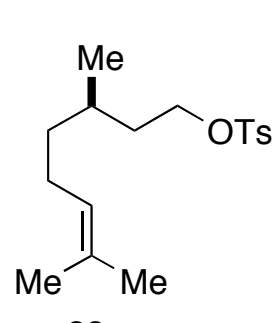

22

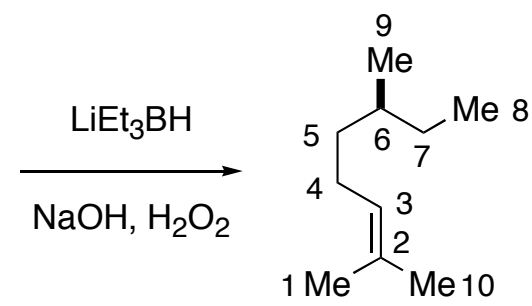

23

A 250-mL, two-necked, round-bottom flask, containing a magnetic stir-bar, septum, and argon inlet was charged with $22(6.9 \mathrm{~g}, 22.1 \mathrm{mmol}, 1.0$ equiv), THF (22 mL). The solution was cooled to $0{ }^{\circ} \mathrm{C}$ using an ice bath. To this solution was added a solution of lithium triethylborohydride (44.2 mL, $44.2 \mathrm{mmol}, 1.0 \mathrm{M}$ in THF, 2.0 equiv) slowly over $15 \mathrm{~min}$. The colorless solution was stirred at room $0{ }^{\circ} \mathrm{C}$ for $3 \mathrm{~h}$. Then $\mathrm{H}_{2} \mathrm{O}(3.0 \mathrm{~mL})$ was added drop wise at 0 ${ }^{\circ} \mathrm{C}$, followed by $3 \mathrm{~N} \mathrm{NaOH}(30 \mathrm{~mL})$. Then $30 \% \mathrm{H}_{2} \mathrm{O}_{2}(30 \mathrm{~mL})$ was added slowly, at a rate that the internal temperature did not rise above $40{ }^{\circ} \mathrm{C}(\sim 2.5 \mathrm{~h})$. After the quench, the biphasic reaction mixture was diluted with pentane $(50 \mathrm{~mL})$ and transferred to a $500-\mathrm{mL}$ separatory funnel and the aqueous layer was extracted. The aqueous phase was back-extracted with pentane $(5 \times 30 \mathrm{~mL})$, the organic layers were combined, washed with water $(4 \times 20 \mathrm{~mL})$ and brine $(20$ $\mathrm{mL})$. The organic layer was dried $\left(\mathrm{Na}_{2} \mathrm{SO}_{4}\right)$, filtered and the pentane was removed by atmospheric distillation. The residue was purified by distillation first at $100 \mathrm{mmHg}$ (oil bath 50 $\left.{ }^{\circ} \mathrm{C}\right)$ to remove the THF and then at $30 \mathrm{mmHg}$ to afford $2.7 \mathrm{~g}(87 \%)$ of $\mathbf{2 3}$ as a colorless liquid.

\section{Data for 23:}

bp: $\quad 76-80{ }^{\circ} \mathrm{C}$ at $30 \mathrm{mmHg}$

${ }^{1}$ H NMR: $\quad\left(500 \mathrm{MHz}, \mathrm{CDCl}_{3}\right)$

5.11 (tq, $J=7.3,1.5 \mathrm{~Hz}, 1 \mathrm{H}, \mathrm{CH}(3)) ; 1.87-2.06\left(\mathrm{~m}, 2 \mathrm{H}, \mathrm{CH}_{2}(4)\right) ; 1.69(\mathrm{~s}, 3 \mathrm{H}$, $\mathrm{CH}_{3}(1$ or 10$\left.)\right) ; 1.61\left(\mathrm{~s}, 3 \mathrm{H}, \mathrm{CH}_{3}(1\right.$ or 10$\left.)\right) ; 1.28-1.38\left(\mathrm{~m}, 3 \mathrm{H}, \mathrm{CH}_{2}(5), \mathrm{CH}(6)\right)$; 1.08-1.18 (m, 2H, $\left.\mathrm{CH}_{2}(7)\right)$; 0.86 (t, $\left.J=7.2 \mathrm{~Hz}, 3 \mathrm{H}, \mathrm{CH}_{3}(8)\right) ; 0.86$ (d, $J=6.4 \mathrm{~Hz}$, $\left.3 \mathrm{H}, \mathrm{CH}_{3}(9)\right)$ 
${ }^{13} \mathrm{C} \mathrm{NMR}: \quad\left(126 \mathrm{MHz}, \mathrm{CDCl}_{3}\right)$

130.97 (C(2)); 125.07 (C(3)); 36.70 (7)); 34.00 (4)); 29.39 (6)); $25.74(5)) ; 25.57$

(1 or 10$)) ; 19.08(9)) ; 17.61$ (1 or 10$)) ; 11.38(\mathrm{C}(8))$

IR: (neat)

2964 (s); 2916 (s); 2875 (s); 2857 (s); 1461 (m); 1377 (m); 1155 (w); 1116 (w); $1090(\mathrm{w}) ; 948(\mathrm{w})$

MS: $\quad(\mathrm{EI})$

140 (( $\left.\mathrm{M}^{+}\right)$30); 84 (17); 83 (21); 70 (64); 69 (100); 57 (27); 56 (39); 55 (48)

Opt. rot. : $\quad[\alpha]_{\mathrm{D}}^{24} 10.8\left(\mathrm{CHCl}_{3}, \mathrm{c}=2.00\right)$

Analysis: $\quad \mathrm{C}_{10} \mathrm{H}_{20}(140.27)$
Calcd:
C, 85.63\%;
H, $14.37 \%$
Found:
C, 85.67\%;
$\mathrm{H}, 14.33 \%$

\section{Preparation of Ethyl $(S)-2,8-D i m e t h y l-2(E), 4(E, Z)$-decadienoate (24) [CSR-VIII-5]}

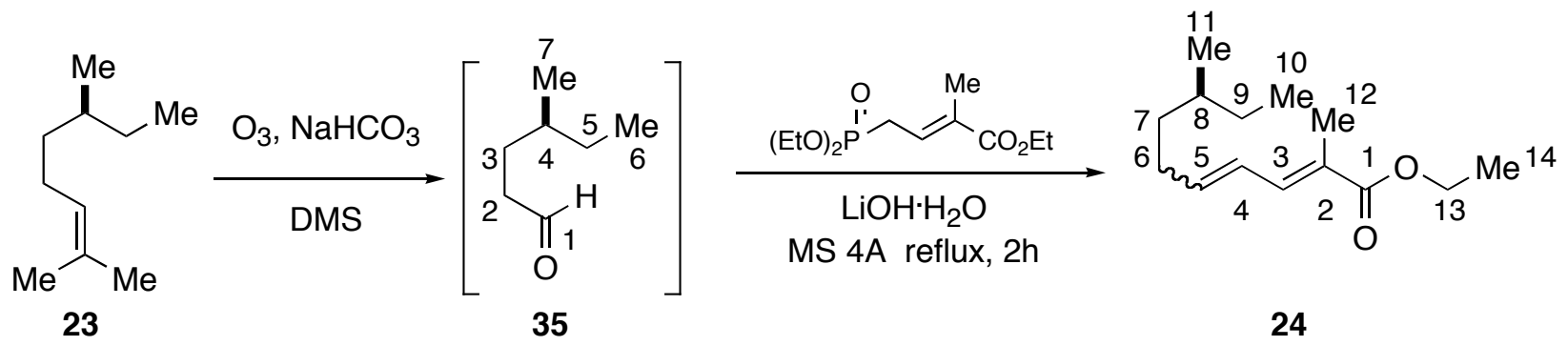

A 25-mL, round-bottomed flask, containing a stir-bar, Teflon thermometer adaptor and gas dispersion tube through the Teflon adaptor was charged with 23 (1.36 g, 9.7 mmol, 1.0 equiv), $\mathrm{CH}_{2} \mathrm{Cl}_{2}(5 \mathrm{~mL})$ and $\mathrm{MeOH}(1.2 \mathrm{~mL})$. To this solution was added $\mathrm{NaHCO}_{3}(162 \mathrm{mg}, 1.9$ mmol, 0.2 equiv). The contents were cooled to $-78{ }^{\circ} \mathrm{C}$ in a 2 -propanol/dry ice bath whereupon ozone was bubbled through the solution for $20 \mathrm{~min}$ or until the contents turned deep blue. Then, oxygen was bubbled through the solution until the blue color dissipated. While still at $-78{ }^{\circ} \mathrm{C}$ (under an argon atmosphere) dimethyl sulfide (1.2 mL, $16.5 \mathrm{mmol}, 1.7$ equiv) was added and the solution was stirred at this temperature for $40 \mathrm{~min}$. The 2-propanol/dry ice bath was removed and the contents were allowed to warm to room temperature over $2.5 \mathrm{~h}$. The colorless solution was filtered though Celite $(2 \mathrm{~g})$ washing the pad with $\mathrm{CHCl}_{3}(5 \mathrm{~mL})$ and pentane $(20 \mathrm{~mL})$. The solvent was removed using rotary evaporation with the water bath cooled to $0{ }^{\circ} \mathrm{C}$. The colorless 
oil was used in the next step without further purification.

The above oil (1.12 g, $9.7 \mathrm{mmol}, 1.0$ equiv, assuming quantitative yield in the first step) was transferred to a 50-mL, one-necked, round-bottomed flask and then THF (10 mL) and a magnetic stir-bar were added. To this solution was added $3 \AA$ molecular sieves $(9 \mathrm{~g}, 1 \mathrm{~g} / \mathrm{mmol})$ and lithium hydroxide monohydrate (448 $\mathrm{mg}, 10.7 \mathrm{mmol}, 1.1$ equiv). The flask was equipped with a water condenser and an argon inlet with a rubber septum. The apparatus was then flushed with argon. To a separate $25-\mathrm{mL}$, one-necked, conical-flask sealed with a septum and flushed with argon, ethyl 4-(diethoxyphosphinyl)tiglate ( $2.82 \mathrm{~g}, 10.7 \mathrm{mmol}, 1.1$ equiv) was added along with THF $(8 \mathrm{~mL})$. The phosphonate solution was transferred via cannula into the mixture of aldehyde 35 rinsing the flask with THF $(0.5 \mathrm{~mL})$. The contents were heated to reflux (oil bath temperature $75^{\circ} \mathrm{C}$ ) and stirred for $2.5 \mathrm{~h}$. The contents became yellow and turbid as the reaction progressed. Upon cooling to room temperature the mixture was filtered thorough Celite $(2.5 \mathrm{~g})$ washing the pad with 1:1 hexane/EtOAc $(40 \mathrm{~mL})$. The crude product was analyzed by ${ }^{1} \mathrm{H}-\mathrm{NMR}$ to reveal a 91:9 ratio of $(E, E)-\mathbf{2 4}$ and $(E, Z)-\mathbf{2 4}$ isomers. The orange slurry was purified by column chromatography (( $\left.\mathrm{SiO}_{2}, 40 \times 220 \mathrm{~mm}\right)$, hexane/EtOAc, 15/1) to afford $1.69 \mathrm{~g}(78 \%)$ of an inseparable mixture of esters $\mathbf{2 4}$ as a colorless oil.

Data for 24:

${ }^{1}$ H NMR: $\quad\left(500 \mathrm{MHz}, \mathrm{CDCl}_{3}\right)$

$9.77(\mathrm{~d}, J=1.7 \mathrm{~Hz}, 1 \mathrm{H}, \mathrm{CH}(1)) ; 2.48-2.36\left(\mathrm{~m}, 2 \mathrm{H}, \mathrm{CH}_{2}(2)\right) ; 1.70-1.63(\mathrm{~m}, 1 \mathrm{H}$, $\mathrm{CH}_{2}(3 \mathrm{a}$ or $\left.3 \mathrm{~b})\right) ; 1.47-1.40\left(\mathrm{~m}, 1 \mathrm{H}, \mathrm{CH}_{2}(3 \mathrm{a}\right.$ or $\left.3 \mathrm{~b})\right) ; 1.40-1.31\left(\mathrm{~m}, 2 \mathrm{H}, \mathrm{CH}_{2}(5 \mathrm{a}\right.$ or $5 \mathrm{~b}, 4)$ ); $1.20-1.12$ (m, 1H, $\mathrm{CH}_{2}(5 \mathrm{a}$ or $5 \mathrm{~b})$ ); 0.87 (t, $\left.J=7.4 \mathrm{~Hz}, 3 \mathrm{H}, \mathrm{CH}_{3}(6)\right) ; 0.86$ (d, $\left.J=6.5 \mathrm{~Hz}, 3 \mathrm{H}, \mathrm{CH}_{3}(7)\right)$

${ }^{13}$ C NMR: $\quad\left(126 \mathrm{MHz}, \mathrm{CDCl}_{3}\right)$

203.27 (C(1)); $41.71(\mathrm{C}(2)) ; 33.96$ (C(4)); 29.13 (C(5)); 28.45 (C(3)); 18.84 $(\mathrm{C}(7)) ; 11.26(\mathrm{C}(6))$

IR: (neat)

2867 (s); $2700(\mathrm{~s}) ; 1725$ (s)

MS: (ESI)

$114\left(\left(\mathrm{M}^{+}\right)\right.$2); 86 (65); 70 (100); 67 (14); 58 (19); 57 (42); 55 (55)

Opt. rot. : $\quad[\alpha]_{\mathrm{D}}^{24} 7.61$ (heptane, $\left.\mathrm{c}=1.29\right)$

HRMS: $\quad\left(\right.$ for $\mathrm{C}_{17} \mathrm{H}_{14} \mathrm{O}$ ) 
Calcd: $\quad 114.1045$

Found: $\quad 114.1043$

Data for 24:

bp: $\quad 100{ }^{\circ} \mathrm{C}$ at $1 \times 10^{-5} \mathrm{mmHg}(\mathrm{ABT})$

${ }^{1}$ H NMR: $\quad\left(500 \mathrm{MHz}, \mathrm{CDCl}_{3}\right)$

7.50 (dt, $J=11.9,1.2 \mathrm{~Hz}, 1 \mathrm{H}, \mathrm{CH}(3),(Z$-isomer)); 7.16 (d, $J=11.5 \mathrm{~Hz}, 1 \mathrm{H}$, $\mathrm{CH}(3),(E$-isomer)); 6.34 (ddt, $J=14.4,11.6$, and $1.4 \mathrm{~Hz}, 1 \mathrm{H}, \mathrm{CH}(4),(E$-isomer)); 6.27 (ddt, $J=11.6,11.2$, and $1.4 \mathrm{~Hz}, 1 \mathrm{H}, \mathrm{CH}(4),(Z$-isomer)); 6.08 (dt, $J=14.4$, $7.2 \mathrm{~Hz}, 2 \mathrm{H}, \mathrm{CH}_{2}(5),(E$-isomer)); 5.83 (dt, $J=11.2,7.2 \mathrm{~Hz}, 1 \mathrm{H}, \mathrm{CH}(5),(Z-$ isomer)); 4.22 (q, $J=7.2 \mathrm{~Hz}, 2 \mathrm{H}, \mathrm{CH}_{2}(13),(Z$-isomer)); 4.20 (q, $J=7.2 \mathrm{~Hz}, 2 \mathrm{H}$, $\mathrm{CH}_{2}$ (13), (E-isomer)); 2.34-2.10 (m, 2H, $\mathrm{CH}_{2}(6),(Z$ and $E$ - isomer)); 1.93 (d, $J=$ $0.8 \mathrm{~Hz}, 3 \mathrm{H}, \mathrm{CH}_{3}(12)$, (Z-isomer)); 1.92 (d, $J=0.8 \mathrm{~Hz}, 3 \mathrm{H}, \mathrm{CH}_{3}(12)$, (E-isomer)); 1.30 (t, $J=7.0 \mathrm{~Hz}, 3 \mathrm{H}, \mathrm{CH}_{3}(14),\left(E\right.$-isomer)); $1.49-1.10$ (m, $5 \mathrm{H}, \mathrm{CH}_{2}(7,9)$ and $\mathrm{CH}(8),(Z$ and $E$ - isomer)); 0.88 (d, $J=6.0 \mathrm{~Hz}, 3 \mathrm{H}, \mathrm{CH}(11),(E$-isomer)); 0.87 (t, $J=8.0 \mathrm{~Hz}, 3 \mathrm{H}, \mathrm{CH}(10),(E$-isomer $))$

\section{${ }^{13} \mathrm{C}$ NMR: $\quad\left(126 \mathrm{MHz}, \mathrm{CDCl}_{3}\right)$}

168.66 (C(1), ( $E$-isomer)); 143.42 (C(5), ( $E$-isomer)); 140.21 (C(3), (Z-isomer)); 138.58 (C(3), (E-isomer)); 132.81 (C(5), (Z-isomer)); 125.77 (C(4), ( $E$-isomer)); 124.98 (C(2), (E-isomer)); 123.63 (C(4), (Z-isomer)); 60.50 (C(13), (Z-isomer)); 60.40 (C(13), (E-isomer)); 36.24 (C(7), (Z-isomer)); 35.72 (C(7), (E-isomer)); 33.94 (C(8), (E-isomer)); 30.89 (C(6), $(E$-isomer)); 29.32 (C(9), $(E$-isomer)); 18.98 (C(11), (E-isomer)); 14.32 (C(12), (E-isomer)); 12.53 (C(14), (E-isomer)); 11.29 (C(10), (E-isomer))

IR: (neat)

3032 (w); 2961(s); 2922 (s); 2856 (m); 1706 (s); 1639 (s); 1610 (w); 1462 (m); 1367 (m); $1284(\mathrm{~m}) ; 1241$ (s); $1166(\mathrm{w}) ; 1105$ (s); 972 (s)

MS: $\quad(\mathrm{EI}, 70 \mathrm{eV})$

224 ((M+ (95); 195 (11); 180 (12); 179 (80); 168 (25); 167 (32); 150 (16); 149 (20); 141 (96); 140 (60); 139 (100); 128 (54); 126 (22); 125 (36); 122 (28); 113 (61); 111 (95); 110 (54); 102 (21); 100 (20); 97 (36); 91 (30); 79 (61); 69 (25); 57 (33) 
TLC: $\quad R_{f} 0.39\left(10: 1\right.$ hexanes/EtOAc) $\left[\mathrm{SiO}_{2}, \mathrm{UV}\right]$

Analysis: $\quad \mathrm{C}_{14} \mathrm{H}_{24} \mathrm{O}_{2}(224.43)$

Calcd: $\quad$ C, $74.95 \% ; \quad H, 10.78 \%$

Found: $\quad$ C, $75.00 \% ; \quad H, 10.79 \%$

\section{Preparation of (S)-2,8-Dimethyldeca-2E,4E-dien-ol (25) ${ }^{18}$ [CSR-VIII-8]}

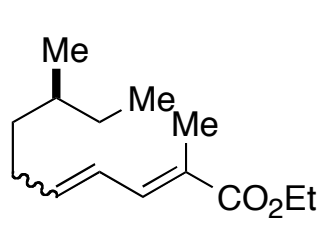

24

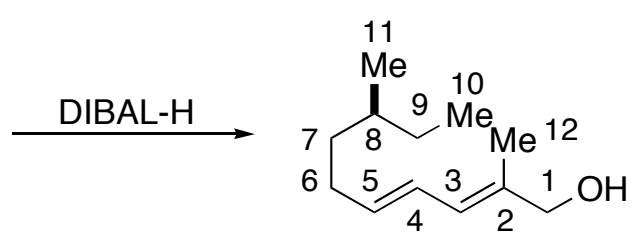

25

A 100-mL, one-necked, recovery-flask, containing a magnetic stir-bar and an argon inlet with a septum, was charged with ester 24 (1.54 g, $6.86 \mathrm{mmol}, 1.0$ equiv) and THF (15 mL). The solution was cooled to $0{ }^{\circ} \mathrm{C}$ using an ice bath. Once at $0{ }^{\circ} \mathrm{C}$, a solution of DIBAL-H $(15.1 \mathrm{~mL}$, $15.1 \mathrm{mmol}, 1.0 \mathrm{M}$ in hexane, 2.2 equiv) was added drop wise over $15 \mathrm{~min}$. The yellow solution quickly became colorless with each drop of DIBAL-H. After the addition ( $\sim 15 \mathrm{~min})$ Celite ( $3 \mathrm{~g})$ and $\mathrm{Et}_{2} \mathrm{O}(15 \mathrm{~mL})$ were added and then $\mathrm{H}_{2} \mathrm{O}(\sim 1.2 \mathrm{~mL})$ was added slowly until a gelatinous solid formed. Diethyl ether $(10 \mathrm{~mL})$ was added and the contents were stirred vigorously to break up the gelatinous solid. The contents were filtered through Celite $(1 \mathrm{~g})$ washing the pad with $\mathrm{Et}_{2} \mathrm{O}$ $(100 \mathrm{~mL})$, and the filtrate was concentrated to a light-yellow oil. The crude product was purified by column chromatography $\left(\left(\mathrm{SiO}_{2}, 40 \times 220 \mathrm{~mm}\right)\right.$, hexanes/EtOAc, 10/1) to afford $1.06 \mathrm{~g}(85 \%$, $(E, E)-\mathbf{2 5})$ and $0.013 \mathrm{~g}(11 \%,(E, Z)-25)$ as colorless oils.

\section{Data for $(\boldsymbol{E}, \boldsymbol{E})-\mathbf{2 5}$ :}

bp: $\quad 95^{\circ} \mathrm{C}\left(8.5 \times 10^{-5} \mathrm{mmHg}, \mathrm{ABT}\right)$

${ }^{1} \mathrm{H} \mathrm{NMR}: \quad\left(500 \mathrm{MHz}, \mathrm{CDCl}_{3}\right)$

6.25 (ddt, $J=10.7,15.1$, and $1.5 \mathrm{~Hz}, 1 \mathrm{H}, \mathrm{CH}(4)) ; 6.02(\mathrm{~d}, J=11.0 \mathrm{~Hz}, 1 \mathrm{H}$, $\mathrm{CH}(3)$ ); 5.70 (dt, $J=15.0,7.1 \mathrm{~Hz}, 1 \mathrm{H}, \mathrm{CH}(5)) ; 4.01\left(\mathrm{~s}, 2 \mathrm{H}, \mathrm{CH}_{2}(1)\right.$ ); 2.18-2.05 (nfoddd, $J=15,9.0$, and $7.3 \mathrm{~Hz}, 2 \mathrm{H}, \mathrm{CH}_{2}(6)$ ); 1.78 (s, 3H, $\mathrm{CH}_{3}(12)$ ); 1.61(s, $1 \mathrm{H}$, br (OH); 1.29-1.45 (m, 3H, CH(8), $\left.\mathrm{CH}_{2}(9)\right) ; 1.11-1.24$ (m, 2H, $\left.\mathrm{CH}_{2}(7)\right) ; 0.86$ (t, J $\left.=7.3 \mathrm{~Hz}, 3 \mathrm{H}, \mathrm{CH}_{3}(10)\right) ; 0.86\left(\mathrm{~d}, J=6.8 \mathrm{~Hz}, 3 \mathrm{H}, \mathrm{CH}_{3}(11)\right)$ 
${ }^{13}$ C NMR: $\quad\left(126 \mathrm{MHz}, \mathrm{CDCl}_{3}\right)$

$135.58(\mathrm{C}(5)) ; 134.60(\mathrm{C}(2)) ; 125.60(\mathrm{C}(4)) ; 125.41(\mathrm{C}(3)) ; 68.75(\mathrm{C}(1)) ; 36.23$ $(\mathrm{C}(7)) ; 33.92(\mathrm{C}(8)) ; 30.54(\mathrm{C}(6)) ; 29.36(\mathrm{C}(9)) ; 19.03(\mathrm{C}(11)) ; 14.08(\mathrm{C}(12))$; $11.33(\mathrm{C}(10))$

IR: (neat)

3325 (s); 2961(s); 2916 (s); 2873 (s); 1461(m); 1377 (m); 1210 (w); 1144 (w); 1067 (w); 1008 (m); 967 (s); $882(\mathrm{w})$

MS: $\quad(\mathrm{EI}, 70 \mathrm{eV})$

182((M+) 100); 111(40); 98(75); 96(67); 95(24); 93(25); 86(33); 85(70); 84(38); 83(67); 82(19); 81(55); 79(34); 71(34); 70(46); 69(67); 67(21); 56 (14); 55(43); 54(29); 53(69); 50(27)

Opt. rot. : $\quad[\alpha]_{\mathrm{D}}^{24} 8.17(\mathrm{EtOH}, \mathrm{c}=1.00)$

TLC: $\quad R_{f} 0.20\left(\mathrm{CH}_{2} \mathrm{Cl}_{2}\right)\left[\mathrm{SiO}_{2}, \mathrm{UV}\right]$

HRMS: for $\left(\mathrm{C}_{12} \mathrm{H}_{22} \mathrm{O}\right)$

Calcd: $\quad 182.1667$

Found: $\quad 182.1671$

Data for $(\boldsymbol{E}, \boldsymbol{E})-\mathbf{2 5}$ :

bp: $\quad 95^{\circ} \mathrm{C}\left(8.5 \times 10^{-5} \mathrm{mmHg}, \mathrm{ABT}\right)$

${ }^{1}$ H NMR: $\quad\left(500 \mathrm{MHz}, \mathrm{CDCl}_{3}\right)$

$6.33(\mathrm{dt}, J=11.2,1.5 \mathrm{~Hz}, 1 \mathrm{H}, \mathrm{CH}(3)) ; 6.02(\mathrm{~d}, J=11.0,1.5 \mathrm{~Hz}, 1 \mathrm{H}, \mathrm{CH}(4)) ; 5.48$ (dt, $J=10.7,7.3 \mathrm{~Hz}, 1 \mathrm{H}, \mathrm{CH}(5)) ; 4.10\left(\mathrm{~d}, J=5.6 \mathrm{~Hz}, 2 \mathrm{H}, \mathrm{CH}_{2}(1)\right) ; 2.16-2.23$ (m, $\left.2 \mathrm{H}, \mathrm{CH}_{2}(6)\right) ; 1.79$ (s, 3H, $\left.\mathrm{CH}_{3}(12)\right)$; 1.32-1.43 (m, 3H, $\left.\mathrm{CH}(8), \mathrm{CH}_{2}(9)\right) ; 1.25$ (s, 1H, (br(OH)); 1.13-1.22 (m, 2H, $\left.\mathrm{CH}_{2}(7)\right) ; 0.87\left(\mathrm{t}, J=7.3 \mathrm{~Hz}, 3 \mathrm{H}, \mathrm{CH}_{3}(10)\right) ; 0.87$ (d, $\left.J=6.1 \mathrm{~Hz}, 3 \mathrm{H}, \mathrm{CH}_{3}(11)\right)$

${ }^{13}$ C NMR: $\quad\left(126 \mathrm{MHz}, \mathrm{CDCl}_{3}\right)$

$137.00(\mathrm{C}(2)) ; 133.17(\mathrm{C}(5)) ; 123.78(\mathrm{C}(4)) ; 120.40$ (C(3)); 69.12 (C(1)); 36.71 $(\mathrm{C}(7)) ; 34.31(\mathrm{C}(8)) ; 29.61(\mathrm{C}(9)) ; 25.51(\mathrm{C}(6)) ; 19.31(\mathrm{C}(11)) ; 14.21(\mathrm{C}(12))$; $11.60(\mathrm{C}(10))$

IR: (neat) 3308(s)；3020(w)；2961(s)；2916(s)；2873(s)；1461(m)；1378(m)；1211(w); 1067(w); 1009(m); 877(w) 
MS: $\quad(E I, 70 \mathrm{eV})$

182((M+ ) 100); 135(29); 123(15); 98(84); 95(59); 85(75); 83(93); 81(64); 79(37);

70(49); 69(86); 67(67); 55(53); 53(96); 50(31)

TLC: $\quad R_{f} 0.18\left(\mathrm{CH}_{2} \mathrm{Cl}_{2}\right)\left[\mathrm{SiO}_{2}, \mathrm{UV}\right]$

HRMS: for $\left(\mathrm{C}_{12} \mathrm{H}_{22} \mathrm{O}\right)$

Calcd: $\quad 182.1667$

Found: $\quad 182.1671$

\section{Preparation of (S)-2,8-Dimethyldeca-2E,4E-dienal (5) ${ }^{18}$ [CSR-VIII-18]}

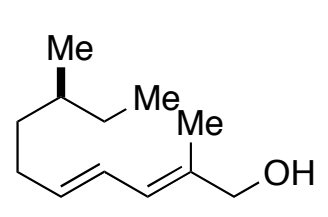

25

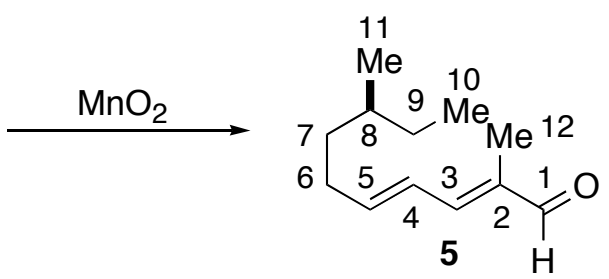

A 100-mL, one-necked, recovery-flask, containing a magnetic stir-bar, water condenser, and an argon inlet with a septum was charged with alcohol 25 (1.07 g, 5.87 mmol, 1.0 equiv), $\mathrm{CHCl}_{3}(23 \mathrm{~mL})$, and manganese dioxide $(2.04 \mathrm{~g}, 23.5 \mathrm{mmol}, 4.0$ equiv). The flask contents were flushed with argon and heated to reflux (oil bath $80{ }^{\circ} \mathrm{C}$ ) with stirring for $4 \mathrm{~h}$. The black suspension was cooled to room temperature and filtered through Celite $(2 \mathrm{~g})$ washing the pad with $\mathrm{CH}_{2} \mathrm{Cl}_{2}(50 \mathrm{~mL})$ to afford a yellow filtrate. The yellow solution was concentrated to a yellow oil which was purified by column chromatography (( $\mathrm{SiO}_{2}, 50$ x $\left.180 \mathrm{~mm}\right)$, hexane/EtOAc, 20/1) to afford $0.943 \mathrm{~g}(89 \%)$ of aldehyde 5 as a light-yellow oil.

Data for 5:

${ }^{1} \mathrm{H} \mathrm{NMR}: \quad\left(500 \mathrm{MHz}, \mathrm{CDCl}_{3}\right)$

9.41(s, 1H, CH(1)); 6.83 (d, $J=11.2 \mathrm{~Hz}, 1 \mathrm{H}, \mathrm{CH}(3)) ; 6.52$ (ddt, $J=15.0,11.1$, and $1.5 \mathrm{~Hz}, 1 \mathrm{H}, \mathrm{CH}(4)) ; 6.24(\mathrm{dt}, J=15.0,7.1 \mathrm{~Hz}, 1 \mathrm{H}, \mathrm{CH}(5)) ; 2.10-2.33(\mathrm{~m}, 2 \mathrm{H}$, $\left.\mathrm{CH}_{2}(6)\right) ; 1.83$ (s, 3H, $\left.\mathrm{CH}_{3}(12)\right) ; 1.43-1.50$ (m, 1H, $\mathrm{CH}(9 \mathrm{a}$ or $9 \mathrm{~b})$ ); 1.32-1.39 (m, $2 \mathrm{H}, \mathrm{CH}(9 \mathrm{a}$ or $9 \mathrm{~b}), \mathrm{CH}(8)) ; 1.24-1.30(\mathrm{~m}, 1 \mathrm{H}, \mathrm{CH}(7 \mathrm{a}$ or $7 \mathrm{~b})) ; 1.13-1.21(\mathrm{~m}, 1 \mathrm{H}$, $\mathrm{CH}(7 \mathrm{a}$ or $7 \mathrm{~b})) ; 0.89\left(\mathrm{~d}, J=6.4 \mathrm{~Hz}, 3 \mathrm{H}, \mathrm{CH}_{3}(11)\right) ; 0.88(\mathrm{t}, J=7.3 \mathrm{~Hz}, 3 \mathrm{H}$, $\left.\mathrm{CH}_{3}(10)\right)$

${ }^{13} \mathrm{C} \mathrm{NMR}: \quad\left(126 \mathrm{MHz}, \mathrm{CDCl}_{3}\right)$

195.23 (C(1)); 149.51 (C(3)); 146.33 (C(5)); 135.83 (C(2)); 125.65 (C(4)); 35.48 
$(\mathrm{C}(7)) ; 33.98(\mathrm{C}(8)) ; 31.10(\mathrm{C}(6)) ; 29.28(\mathrm{C}(9)) ; 18.97$ (C(11)); 11.32 (C(10));

$9.38(\mathrm{C}(12))$

IR: (neat)

3342 (w); 2960 (s); 2926 (s); 2874 (s); 2856 (m); 2709 (w); 2764 (w); 1685 (s);

1636 (s); 1462 (m); 1405 (w); 1378 (w); 1212 (m); 1008 (m); 967 (m); 835 (w); $683(\mathrm{w})$

MS: $\quad(\mathrm{EI}, 70 \mathrm{eV})$

180 ((M+) 9); 110 (16); 97 (21); 95 (100); 81 (15); 79 (10); 67 (11)

Opt. rot. : $\quad[\alpha]_{\mathrm{D}}^{24} 13.58(\mathrm{EtOH} ; \mathrm{c}=0.55)$

TLC: $\quad R_{f} 0.36\left(10: 1\right.$ hexanes/EtOAc) $\left[\mathrm{SiO}_{2}, \mathrm{UV}\right]$

HRMS: (for $\mathrm{C}_{12} \mathrm{H}_{20} \mathrm{O}$ )

Calcd:

180.1515

Found:

180.1514

Preparation of $(4 S, 11 S)$-4-Hydroxy-5,11-Dimethyltrideca-1,5E,7E-triene (27) [CSR-VIII43]
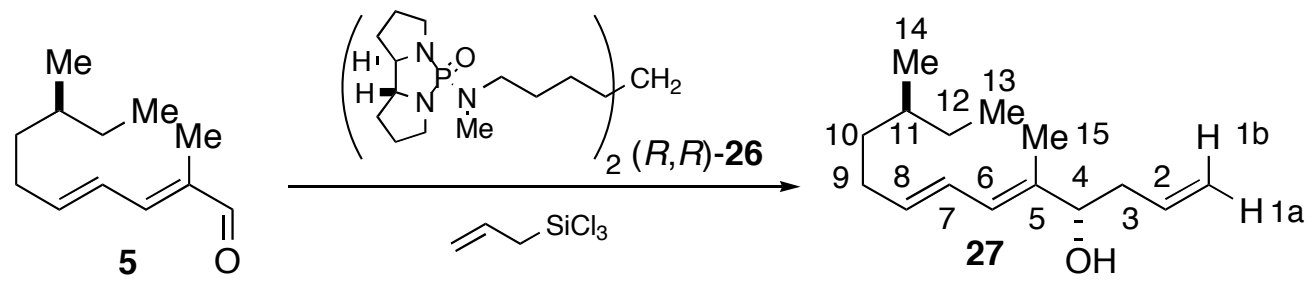

In a 5-mL, round-bottomed flask were placed a magnetic stir-bar, phosphoramide $(R, R)$ 26 (40 mg, $0.1 \mathrm{mmol}, 0.01$ equiv), $\mathrm{CH}_{2} \mathrm{Cl}_{2}(1 \mathrm{~mL})$, and $i-\operatorname{Pr}_{2} \mathrm{NEt}(1 \mathrm{~mL})$. The solution was cooled to $-70{ }^{\circ} \mathrm{C}$ using a 2-propanol/dry ice bath. To the reaction mixture was added allyltrichlorosilane $(290 \mu \mathrm{L}, 2.0 \mathrm{mmol}, 2.0$ equiv) followed by aldehyde 5 (180 mg, $1.0 \mathrm{mmol}$, 1.0 equiv) drop wise. The reaction mixture was stirred at $-70{ }^{\circ} \mathrm{C}$ for $8 \mathrm{~h}$. Then the reaction mixture was transferred via cannula into 50-mL Erlenmeyer flask, containing a vigorously stirring solution of a 1:1 mixture of saturated, aqueous $\mathrm{NaHCO}_{3}$ and saturated, aqueous KF solution $(20 \mathrm{~mL})$, at room temperature. Then the reaction flask was rinsed with $\mathrm{CH}_{2} \mathrm{Cl}_{2}(10 \mathrm{~mL})$ and this wash was transferred via cannula into the Erlenmeyer. The resulting yellow biphasic mixture was stirred vigorously for $12 \mathrm{~h}$ at room temperature. The solution was filtered through a 
layer of Celite $(1 \mathrm{~g})$, and the filtrate was transferred into a $250-\mathrm{mL}$ separatory funnel. The aqueous layer was extracted with EtOAc $(3 \times 20 \mathrm{~mL})$, and the combined organic layers were washed with brine $(20 \mathrm{~mL})$. The organic solution was dried over $\mathrm{Na}_{2} \mathrm{SO}_{4}$ and concentrated under reduced pressure to afford a yellow oil. The crude product was purified using column chromatography (( $\left.\mathrm{SiO}_{2}, 30 \times 200 \mathrm{~mm}\right)$, hexanes/EtOAc, 20/1 to 10/1) to afford $0.195 \mathrm{~g}(88 \%)$ of 27 as a clear, colorless oil.

\section{Data for 27:}

${ }^{1}$ H NMR: $\quad\left(500 \mathrm{MHz}, \mathrm{CDCl}_{3}\right)$

6.24 (ddt, $J=15.0,10.9$, and $1.5 \mathrm{~Hz}, 1 \mathrm{H}, \mathrm{CH}(7)) ; 6.02(\mathrm{~d}, J=10.9 \mathrm{~Hz}, 1 \mathrm{H}$, $\mathrm{CH}(6)$ ); 5.78 (ddt, $J=17.1,10.3$, and $7.3 \mathrm{~Hz}, 1 \mathrm{H}, \mathrm{CH}(2)$ ); 5.68 (dt, $J=16.8,7.3$ $\mathrm{Hz}, 1 \mathrm{H}, \mathrm{CH}(8)$ ); 5.14 (dd, $J=10.3,1.7 \mathrm{~Hz}, 1 \mathrm{H}, \mathrm{CH}(1 \mathrm{a})$ ); 5.10 (dq, $J=16.8,1.5$ $\mathrm{Hz}, 1 \mathrm{H}, \mathrm{CH}(1 \mathrm{~b})) ; 4.08(\mathrm{t}, J=6.8 \mathrm{~Hz}, 1 \mathrm{H}, \mathrm{CH}(4)) ; 2.27-2.40\left(\mathrm{~m}, 2 \mathrm{H}, \mathrm{CH}_{2}(3)\right)$; 2.02-2.20 (m, 2H, $\left.\mathrm{CH}_{2}(9)\right) ; 1.75\left(\mathrm{~s}, 3 \mathrm{H}, \mathrm{CH}_{3}(15)\right) ; 1.62(\mathrm{~d}, J=6.8 \mathrm{~Hz}, 1 \mathrm{H}$, (br(OH)); 1.30-1.44 (m, 3H, CH(11), $\left.\mathrm{CH}_{2}(12)\right) ; 1.12-1.23$ (m, 2H, $\left.\mathrm{CH}_{2}(10)\right) ; 0.86$ (d, $\left.J=6.8 \mathrm{~Hz}, 3 \mathrm{H}, \mathrm{CH}_{3}(14)\right) ; 0.86$ (t, $\left.J=7.1 \mathrm{~Hz}, 3 \mathrm{H}, \mathrm{CH}_{3}(13)\right)$

${ }^{13} \mathrm{C}$ NMR: $\quad\left(126 \mathrm{MHz}, \mathrm{CDCl}_{3}\right)$

$136.30(\mathrm{C}(5)) ; 135.70(\mathrm{C}(8)) ; 134.71(\mathrm{C}(2)) ; 125.63(\mathrm{C}(7)) ; 125.53(\mathrm{C}(6)) ; 117.81$ $(\mathrm{C}(1)) ; 76.20(\mathrm{C}(4)) ; 39.86(\mathrm{C}(3)) ; 36.18$ (C(10)); 33.19 (C(11)); $30.54(\mathrm{C}(9))$; $29.33(\mathrm{C}(12)) ; 19.02(\mathrm{C}(14)) ; 12.28(\mathrm{C}(15)) ; 11.32(\mathrm{C}(13))$

IR: (neat)

3368 (m); 2961 (s); 2917 (s); 2874 (s); 1641 (w); 1461 (w); 1378 (w); 997 (w); $964(\mathrm{w}) ; 911(\mathrm{~m})$

MS: $\quad(E I, 70 \mathrm{eV})$

222 ((M+) 4); 181(100); 163 (22); 121 (10); 111 (30); 97 (42); 95 (19); 93 (62); 91

(13); 83 (63); 81 (35); 79 (16); 71(18); 69 (26); 67 (21); 57 (19); 55 (39)

Opt. Rot.: $\quad[\alpha]_{\mathrm{D}}^{24} 23.22(\mathrm{c}=0.50, \mathrm{EtOH})$

TLC: $\quad R_{f} 0.22$ (hexanes/EtOAc, 10/1) $\left[\mathrm{SiO}_{2}, \mathrm{CAM}\right]$

SFC: (Chiralpak-AD, $3.0 \mathrm{~mL} / \mathrm{min}, 3.0 \% \mathrm{MeOH}, 150 \mathrm{bar}$ );

(4R)-27: $3.22 \min (4.1 \%)$; (4S)-27: $3.50 \min (95.9 \%)$ 
Analysis: $\quad \mathrm{C}_{15} \mathrm{H}_{26} \mathrm{O}(222.37)$

$\begin{array}{lll}\text { Calcd: } & \text { C, } 81.02 \% ; & \text { H, } 11.79 \% \\ \text { Found: } & \text { C, } 80.94 \% ; & \text { H, } 11.81 \%\end{array}$

Determination of the $\mathrm{C}(4)$ Configuration of (4S,11S)-4-Hydroxy-5,11-Dimethyltrideca1,5E,7E-triene (27) [CSR-VIII-29]<smiles>C=CC[C@H](O)/C(C)=C/C=C\CCC(C)CC</smiles>

27

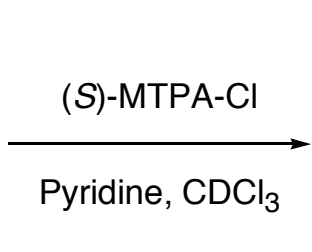

In a 5-mm NMR tube was placed a solution of allyl alcohol $27(4.0 \mathrm{mg}, 0.0180 \mathrm{mmol})$ in $\mathrm{CDCl}_{3}(0.5 \mathrm{~mL})$. To the solution was added sequentially pyridine $(0.2 \mathrm{~mL}, 0.196 \mathrm{mmol}, 11$ equiv) and (S)- $\alpha$-mehoxy- $\alpha$-trifluoromethyl-phenylacetyl chloride (8.4 $\mu \mathrm{L}, 0.045 \mathrm{mmol}, 2.5$ equiv). The reaction mixture was thoroughly mixed and stood for $2 \mathrm{~h}$ at room temperature. The crude Mosher ester $(R)-27$ was analyzed by ${ }^{1} \mathrm{H}-\mathrm{NMR}$ (Table 1 ).

Determination of the $\mathrm{C}(4)$ Configuration of $(4 S, 11 S)-4-H y d r o x y-5,11-D i m e t h y l t r i d e c a-$ 1,5E,7E-triene (27) [CSR-VIII-30]<smiles>C=CC[C@H](O)/C(C)=C/C=C\CCC(C)C</smiles>

27

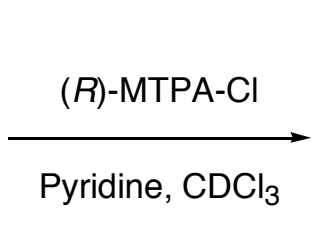

Pyridine, $\mathrm{CDCl}_{3}$

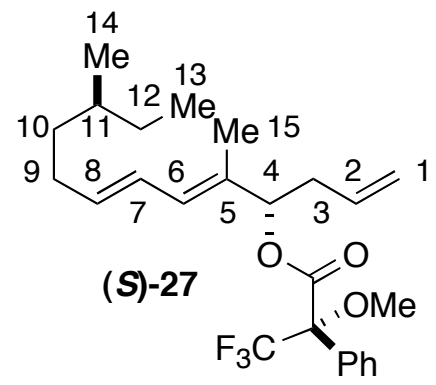

In a 5-mm NMR tube was placed a solution of allyl alcohol $27(4.0 \mathrm{mg}, 0.0180 \mathrm{mmol})$ in $\mathrm{CDCl}_{3}(0.5 \mathrm{~mL})$. To the solution was added sequentially pyridine $(0.2 \mathrm{~mL}, 0.196 \mathrm{mmol}, 11$ equiv) and $(R)$ - $\alpha$-mehoxy- $\alpha$-trifluoromethyl-phenylacetyl chloride $(8.4 \mu \mathrm{L}, 0.045 \mathrm{mmol}, 2.5$ equiv). The reaction mixture was thoroughly mixed and was stood for $2 \mathrm{~h}$ at room temperature. 
The crude Mosher ester $(S)$-27 was analyzed by ${ }^{1} \mathrm{H}-\mathrm{NMR}$ (Table 1$)$.

Table 1: Mosher Ester Analysis. Assignment of C(4) Stereocenter of 27

\begin{tabular}{ccccc}
\hline Assignment & $\delta$-27 & $\delta(S$-ester $)$ & $\delta(R$-ester $)$ & $\Delta \delta-(S-R)$ \\
\hline 7 & 6.243 & 6.154 & 6.108 & 0.046 \\
6 & 6.02 & 6.047 & 5.941 & 0.106 \\
2 & 5.78 & 5.54 & 5.65 & -0.11 \\
8 & 5.68 & 5.683 & 5.615 & 0.068 \\
1 & 5.14 & 4.952 & 5.04 & -0.088 \\
4 & 4.08 & 3.686 & 3.692 & -0.006 \\
3 & $2.27-2.40$ & 2.363 & 2.414 & -0.051 \\
9 & $2.02-2.20$ & 2.057 & 2.048 & 0.009 \\
15 & 1.75 & 1.679 & 1.533 & 0.146 \\
12,11 & $1.30-1.44$ & 1.317 & 1.317 & 0 \\
10 & $1.12-1.23$ & 1.121 & 1.121 & 0 \\
14 & 0.86 & 0.805 & 0.811 & -0.006 \\
\hline
\end{tabular}

\section{Preparation of $(5 S, 12 S)-5-H y d r o x y-6,12-D i m e t h y l t e t r a d e c a-2 E, 6 E, 8 E$-trienal (28) [CSR- VIII-31]}

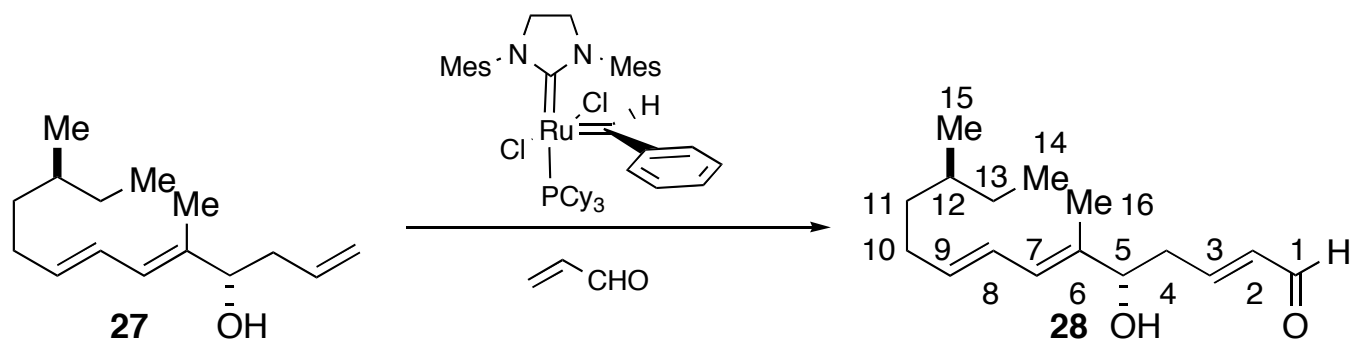

To a 5-mL Schlenk flask, purged with argon, were placed a magnetic stir-bar, Grubbs $2^{\text {nd }}$ generation catalyst (42 mg, $0.05 \mathrm{mmol}, 0.05$ equiv), $\mathrm{CH}_{2} \mathrm{Cl}_{2}(1 \mathrm{~mL})$ and acrolein $(868 \mu \mathrm{L}, 13$ mmol, 13 equiv). To a 5-mL conical-flask, flushed with argon and equipped with a magnetic stirbar and a septum was placed allyl alcohol 27 (222.3 mg, $1.0 \mathrm{mmol}, 1.0$ equiv) along with $\mathrm{CH}_{2} \mathrm{Cl}_{2}$ $(1 \mathrm{~mL})$. The solution of $\mathbf{2 7}$ was transferred via cannula into the flask. Bubbling was observed and the contents were stirred at room temperature for $2 \mathrm{~h}$. The resulting mixture was diluted with hexanes/EtOAc $(3 / 1,10 \mathrm{~mL})$ and immediately subjected to column chromatography $\left(\left(\mathrm{SiO}_{2}, 40 \mathrm{x}\right.\right.$ 200), hexanes/EtOAc, 3/1) to afford $0.223 \mathrm{~g}(90 \%)$ of 28 as a clear, yellow oil.

Data for 28:

bp: $\quad 120^{\circ} \mathrm{C}\left(3.0 \times 10^{-5} \mathrm{mmHg}, \mathrm{ABT}\right)$ 
${ }^{1}$ H NMR: $\quad\left(500 \mathrm{MHz}, \mathrm{CDCl}_{3}\right)$

9.49 (d, $J=8.1 \mathrm{~Hz}, 1 \mathrm{H}, \mathrm{CH}(1)) ; 6.83$ (dt, $J=15.6,7.2 \mathrm{~Hz}, 1 \mathrm{H}, \mathrm{CH}(3)) ; 6.22$ (dd, $J=15.0,10.7 \mathrm{~Hz}, 1 \mathrm{H}, \mathrm{CH}(8)) ; 6.17$ (dd, $J=15.6,7.3 \mathrm{~Hz}, 1 \mathrm{H}, \mathrm{CH}(2)) ; 6.04$ (d, $J$ $=10.7 \mathrm{~Hz}, 1 \mathrm{H}, \mathrm{CH}(7)) ; 5.72(\mathrm{dt}, J=15.0,7.3 \mathrm{~Hz}, 1 \mathrm{H}, \mathrm{CH}(9)) ; 4.22(\mathrm{t}, J=6.4 \mathrm{~Hz}$, 1H, $\mathrm{CH}(5))$; 2.54-2.65 (m, 2H, $\left.\mathrm{CH}_{2}(4)\right) ; 2.05-2.20$ (m, 2H, $\left.\mathrm{CH}_{2}(10)\right) ; 1.78$ (s, $1 \mathrm{H}$, (br(OH)); 1.75 (s, 3H, $\mathrm{CH}_{3}(16)$ ); 1.29-1.43 (m, 3H, CH(12), $\mathrm{CH}_{2}(13)$ ); 1.11-1.23 $\left(\mathrm{m}, 2 \mathrm{H}, \mathrm{CH}_{2}(11)\right) ; 0.86\left(\mathrm{~d}, J=6.4 \mathrm{~Hz}, 3 \mathrm{H}, \mathrm{CH}_{3}(15)\right) ; 0.86(\mathrm{t}, J=7.6 \mathrm{~Hz}, 3 \mathrm{H}$, $\left.\mathrm{CH}_{3}(14)\right)$

${ }^{13} \mathrm{C}$ NMR: $\quad\left(126 \mathrm{MHz}, \mathrm{CDCl}_{3}\right)$

194.34 (C(1)); 154.95 (C(3)); 137.01 (C(9)); 135.73 (C(6)); 134.84 (C(2)); 126.71 $(\mathrm{C}(7)) ; 125.44(\mathrm{C}(8)) ; 76.15(\mathrm{C}(5)) ; 38.44(\mathrm{C}(4)) ; 36.31$ (C(13)); $34.14(\mathrm{C}(12))$; 30.77 (C(10)); 29.54 (C(11)); 19.23 (C(15)); 12.33 (C(16)); 11.53 (C(14))

IR: (neat)

3429 (w); 2960 (m); 2922 (m); 2874 (m); 1691 (s); 1637 (w); 1461 (w); 1378 (w); $1129(\mathrm{w}) ; 1040(\mathrm{w}) ; 1012(\mathrm{w}) ; 967(\mathrm{~m})$

MS: $\quad(\mathrm{EI}, 70 \mathrm{eV})$

250 ( $\mathrm{M}^{+}$(2)); 239 (19); 232 (10); 205 (2); 181 (100); 163 (72); 148 (17); 135 (4); 121 (12); 107 (24); 95 (56); 83 (52); 69 (23); 55 (38)

Opt. Rot.: $\quad[\alpha]_{\mathrm{D}}^{24} 10.55(\mathrm{c}=0.485$, EtOH $)$

TLC: $\quad R_{f} 0.20$ (hexanes/EtOAc, 10/1) $\left[\mathrm{SiO}_{2}, \mathrm{CAM}\right]$

Analysis: $\quad \mathrm{C}_{16} \mathrm{H}_{26} \mathrm{O}_{2}(250.38)$

Calcd: $\quad$ C, $76.75 \% ; \quad H, 10.47 \%$

Found: $\quad$ C, $76.86 \% ; \quad H, 10.74 \%$

Preparation of $(5 S, 12 S)$-6,12-Dimethyl-5-(triethylsilyloxy)tetradeca-2E,6E,8E-trienal (29) [CSR-VIII-68]

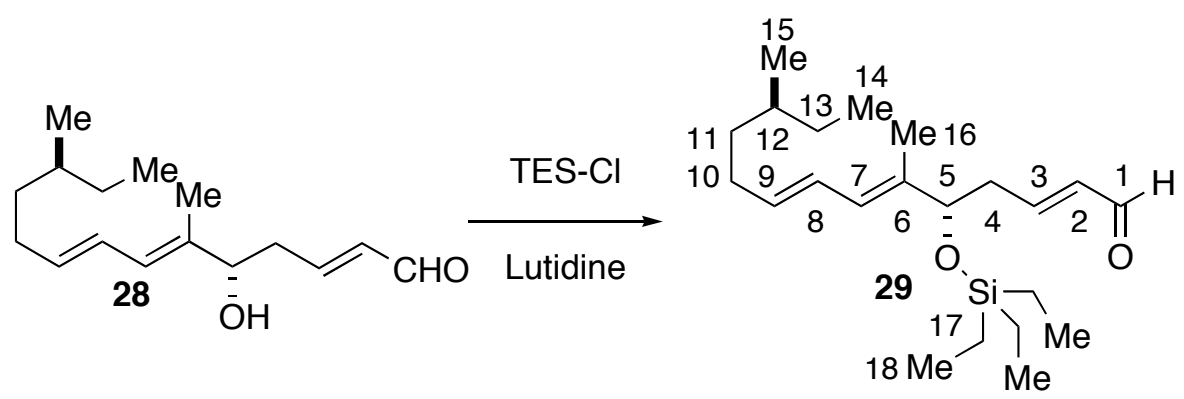


To a 35-mL, one-neck, round-bottom flask, equipped with an argon inlet with a septum, were placed aldehyde 28 (258 mg, $1.03 \mathrm{mmol}, 1.0$ equiv), $\mathrm{CH}_{2} \mathrm{Cl}_{2}(12 \mathrm{~mL})$, lutidine (360 $\mu \mathrm{L}, 6.0$ mmol, 6.0 equiv), and triethylsilyl chloride ( $519 \mu \mathrm{L}, 3.0 \mathrm{mmol}, 3.0$ equiv). The yellow solution was stirred for $7 \mathrm{~h}$. The contents were quenched with saturated, aqueous sodium bicarbonate (20 $\mathrm{mL}$ ) and transferred into a $250-\mathrm{mL}$ separatory funnel. The aqueous layer was extracted with $\mathrm{CH}_{2} \mathrm{Cl}_{2}(3 \times 10 \mathrm{~mL})$, and the combined organic extracts were washed with water $(3 \times 20 \mathrm{~mL})$ and brine $(20 \mathrm{~mL})$. The organic extracts was dried over $\mathrm{Na}_{2} \mathrm{SO}_{4}$ and concentrated to a yellow oil. The crude product was purified using column chromatography $\left(\left(\mathrm{SiO}_{2}, 30\right.\right.$ x $\left.200 \mathrm{~mm}\right)$, hexanes/EtOAc, 10/1 to 3/1) to afford $0.342 \mathrm{~g}(92 \%)$ of 29 as a clear, yellow oil.

Data for 29:

${ }^{1} \mathrm{H}$ NMR: $\quad\left(500 \mathrm{MHz}, \mathrm{CDCl}_{3}\right)$

9.48 (d, $J=7.8 \mathrm{~Hz}, 1 \mathrm{H}, \mathrm{CH}(1)) ; 6.80$ (dt, $J=15.6,8.1 \mathrm{~Hz}, 1 \mathrm{H}, \mathrm{CH}(3)) ; 6.20$ (dd, $J=15.0,10.7 \mathrm{~Hz}, 1 \mathrm{H}, \mathrm{CH}(8)) ; 6.13(\mathrm{dd}, J=15.6,8.0 \mathrm{~Hz}, 1 \mathrm{H}, \mathrm{CH}(2)) ; 5.96$ (d, $J=$ $10.8 \mathrm{~Hz}, 1 \mathrm{H}, \mathrm{CH}(7)) ; 5.67$ (dt, $J=15.0,7.3 \mathrm{~Hz}, 1 \mathrm{H}, \mathrm{CH}(9)) ; 4.16$ (t, $J=5.4 \mathrm{~Hz}$, $1 \mathrm{H}, \mathrm{CH}(5)) ; 2.46-2.61\left(\mathrm{~m}, 2 \mathrm{H}, \mathrm{CH}_{2}(4)\right) ; 2.04-2.18$ (m, 2H, $\left.\mathrm{CH}_{2}(10)\right) ; 1.71$ (s, 3H, $\left.\mathrm{CH}_{3}(16)\right)$; 1.29-1.45 (m, 3H, CH(12), $\left.\mathrm{CH}_{2}(13)\right)$; 1.12-1.25 (m, 3H, $\mathrm{CH}_{3}(11)$ ); 0.93 $\left(\mathrm{t}, J=8.0 \mathrm{~Hz}, 3 \mathrm{H} \mathrm{CH}_{3}(18)\right) ; 0.86\left(\mathrm{~d}, J=6.0 \mathrm{~Hz}, 3 \mathrm{H} \mathrm{CH}_{3}(15)\right) ; 0.86(\mathrm{t}, J=7.3 \mathrm{~Hz}$, $\left.3 \mathrm{H}, \mathrm{CH}_{3}(14)\right) ; 0.57$ (q, $\left.J=8.0 \mathrm{~Hz}, 2 \mathrm{H}, \mathrm{CH}_{2}(17)\right)$

${ }^{13} \mathrm{C} \mathrm{NMR}: \quad\left(126 \mathrm{MHz}, \mathrm{CDCl}_{3}\right)$

194.31 (C(1)); 155.75 (C(3)); 136.19 (C(6)); 135.99 (C(7)); 134.66 (C(9)); 126.12 $(\mathrm{C}(2)) ; 125.66$ (C(8)); 76.87 (C(5)); 40.25 (C(4)); 36.40 (C(13)); 34.27 (C(12)); 30.83 (C(10)); 29.60 (C(11)); 19.31 (C(15)); 12.18 (C(16)); 11.58 (C(14)); 7.06 $(\mathrm{C}(18)) ; 4.97$ (C(17))

IR: (neat) 2957 (s); 2913 (s); 2876 (s); 1697 (s); 1638 (s); 1460 (m); 1378 (w); 1333 (w); 1239 (w); $1128(\mathrm{w}) ; 1069$ (m); 1006 (s); 971(s); 743 (s)

MS: $\quad(\mathrm{EI}, 70 \mathrm{eV})$

$364\left(\mathrm{M}^{+}\right)$1)); 295 (100); 225 (8); 211 (3); 185 (3); 157 (10); 115 (53); 103 (14); 87 (59); 59 (20)

Opt. Rot.: $\quad[\alpha]_{\mathrm{D}}^{24} 9.49(\mathrm{c}=0.575, \mathrm{EtOH})$

TLC: $\quad R_{f} 0.44$ (hexanes/EtOAc, $\left.10 / 1\right)\left[\mathrm{SiO}_{2}, \mathrm{CAM}\right]$ 
Analysis: $\quad \mathrm{C}_{22} \mathrm{H}_{40} \mathrm{O}_{2} \mathrm{Si}(318.48)$
Calcd:
C, $72.47 \%$
$\mathrm{H}, 11.06 \%$
Found:
C, $72.63 \%$;
$\mathrm{H}, 8.41 \%$

\section{Preparation of Methyl (7S,14S)-8,14-Dimethyl-7-(triethylsilyloxy)hexadeca-2E,4E,8E,10E- tetraenoate (36) [CSR-VIII-77]}

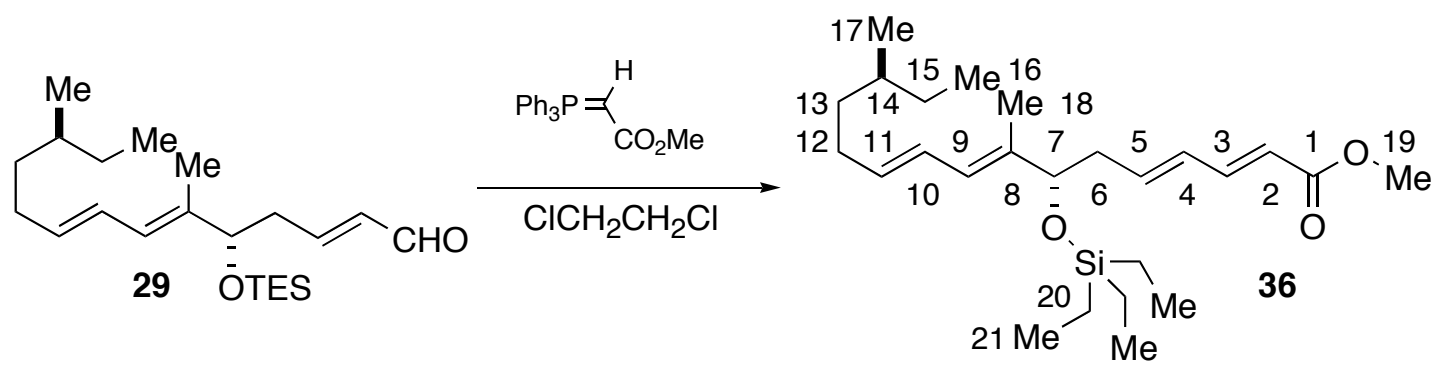

To a 50-mL, one-necked, round-bottom flask, equipped with a magnetic stir-bar, argon inlet with a septum, and water condenser, were placed aldehyde 29 (365 mg, $1.00 \mathrm{mmol}, 1.0$ equiv), 1,2-dichloroethane (5 mL). To a 5-mL one-necked, conical-flask, sealed with a septum, methyl (triphenylphosphoranylidene)acetate $(501 \mathrm{mg}, 1.5 \mathrm{mmol}, 1.5 \mathrm{equiv})$ was added along with dichloroethane $(4 \mathrm{~mL})$. The solution of ylide was transferred via cannula into the solution of aldehyde using dichloroethane $(1 \mathrm{~mL}$ ) to rinse. The contents were heated to reflux (oil bath temperature was $95^{\circ} \mathrm{C}$ ) and stirred for $18 \mathrm{~h}$. Upon cooling to room temperature the orange solution was transferred to a $125-\mathrm{mL}$ separatory funnel containing $30 \mathrm{~mL}$ of water. The aqueous layer was extracted with $\mathrm{CH}_{2} \mathrm{Cl}_{2}(3 \times 15 \mathrm{~mL})$, and the combined organic extracts were washed with brine $(10 \mathrm{~mL})$. The organic extracts was dried over $\mathrm{Na}_{2} \mathrm{SO}_{4}$, filtered, and concentrated to an orange syrup. The crude product was analyzed by ${ }^{1} \mathrm{HNMR}$ to reveal a 90:10 ratio of $(E, E, E, E)$ 36 to $(E, E, E, Z)-36$ isomers. The crude product was purified with column chromatography (( $\left.\mathrm{SiO}_{2}, 40 \times 200 \mathrm{~mm}\right)$, hexane $/ \mathrm{CH}_{2} \mathrm{Cl}_{2}, 2 / 1$ to $\left.1 / 1\right)$ to afford $0.379 \mathrm{~g}(90 \%)$ of $\left.2(E)-36\right)$ and 0.029 $\mathrm{g}(7 \%)$ of $2(Z)-36$ as a clear, yellow oils.

\section{Data for $(E, E, E, E)-\mathbf{3 6}$ :}

${ }^{1} \mathrm{H}$ NMR: $\quad\left(500 \mathrm{MHz}, \mathrm{CDCl}_{3}\right)$

$7.25(\mathrm{dd}, J=15.4,10.7 \mathrm{~Hz}, 1 \mathrm{H}, \mathrm{CH}(3)) ; 6.20(\mathrm{dd}, J=14.9,10.7 \mathrm{~Hz}, 1 \mathrm{H}$, $\mathrm{CH}(10)) ; 6.18(\mathrm{dd}, J=15.3,11.0 \mathrm{~Hz}, 1 \mathrm{H}, \mathrm{CH}(4)) ; 6.06$ (dt, $J=15.3,7.6 \mathrm{~Hz}, 1 \mathrm{H}$, $\mathrm{CH}(5)) ; 5.91$ (d, $J=15.4 \mathrm{~Hz}, 1 \mathrm{H}, \mathrm{CH}(9)) ; 5.79$ (d, $J=15.4 \mathrm{~Hz}, 1 \mathrm{H}, \mathrm{CH}(2)) ; 5.65$ $(\mathrm{dt}, J=14.9,7.0 \mathrm{~Hz}, 1 \mathrm{H}, \mathrm{CH}(11)) ; 4.05$ (t, $J=5.9 \mathrm{~Hz}, 1 \mathrm{H}, \mathrm{CH}(7)) ; 3.76$ (s, 3H, 
$\left.\mathrm{CH}_{3}(19)\right) ; 2.30-2.43\left(\mathrm{~m}, 2 \mathrm{H}, \mathrm{CH}_{2}(6)\right) ; 2.20-2.02\left(\mathrm{~m}, 2 \mathrm{H}, \mathrm{CH}_{2}(12)\right) ; 1.69$ (s, 3H, $\left.\mathrm{CH}_{3}(18)\right) ; 1.45-1.30$ (m, 3H, CH(14), $\left.\mathrm{CH}_{2}(15)\right) ; 1.25-1.12$ (m, 2H, $\left.\mathrm{CH}_{2}(13)\right) ; 0.90$ (t, $\left.J=8.1 \mathrm{~Hz}, 9 \mathrm{H}, \mathrm{CH}_{3}(21)\right) ; 0.86\left(\mathrm{~d}, J=7.0 \mathrm{~Hz}, 3 \mathrm{H}, \mathrm{CH}_{3}(17)\right) ; 0.86(\mathrm{t}, J=6.8$ $\left.\mathrm{Hz}, 3 \mathrm{H}, \mathrm{CH}_{3}(16)\right) ; 0.55$ (q, $\left.J=8.1 \mathrm{~Hz}, 6 \mathrm{H}, \mathrm{CH}_{2}(20)\right)$

${ }^{13} \mathrm{C} \mathrm{NMR}: \quad\left(126 \mathrm{MHz}, \mathrm{CDCl}_{3}\right)$

168.01 (C(1)); 145.43 (C(3)); 141.48 (C(5)); 136.93 (C(8)); 135.49 (C(11)); 130.24 (C(4)); 125.84 (C(10)); 125.74 (C(9)); 119.27 (C(2)); 77.68 (C(7)); 51.72 (C(19)); 40.68 (C(6)); 36.45 (C(13)); 34.28 (C(14)); 30.84 (C(12)); 29.61 (C(15)); 19.33 (C(17)); 12.12 (C(18)); 11.59 (C(16)); 7.07 (C(21)); 4.75 (C(20))

IR: (neat)

2956 (s); 2913 (s); 2876 (s); 1723 (s); 1645 (s); 1617 (w); 1459 (m); 1435 (w); 1345 (w); 1302 (w); 1262 (s); 1208 (m); 1135 (m); 1067 (s); 1002 (s); 965 (m); $743(\mathrm{~m})$

MS: $\quad(\mathrm{EI}, 70 \mathrm{eV})$

390 (M+-OMe, 1); 295 (100); 281(2); 225 (4); 171 (4); 115 (28); 87 (36); 59 (14)

Opt. Rot.: $\quad[\alpha]_{\mathrm{D}}^{24} 12.62\left(\mathrm{c}=0.530, \mathrm{CHCl}_{3}\right)$

TLC: $\quad R_{f} 0.47$ (hexanes/EtOAc, $\left.10 / 1\right)\left[\mathrm{SiO}_{2}, \mathrm{UV}\right]$

Analysis: $\quad \mathrm{C}_{25} \mathrm{H}_{44} \mathrm{O}_{3} \mathrm{Si}(420.70)$

Calcd: $\quad$ C, $71.37 \%$; $\quad$ H, 10.54\%

Found: $\quad$ C, $71.45 \%$; $\quad H, 10.90 \%$

Preparation of $(7 S, 14 S)-8,14-D i m e t h y l-7-(t r i e t h y l s i l y l o x y) h e x a d e c a-2 E, 4 E, 8 E, 10 E-$ tetraenoic Acid (2) [CSR-IX-57] ${ }^{18}$
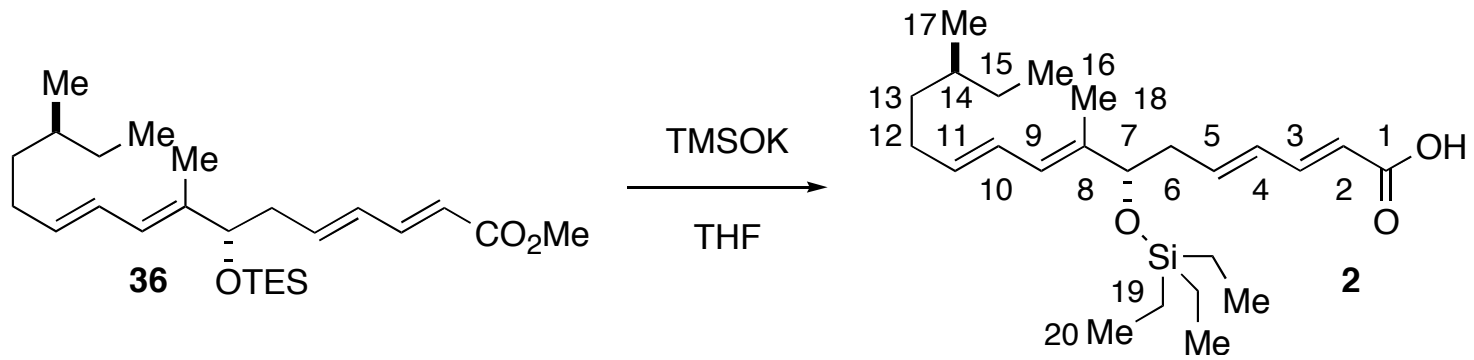

To a 25-mL, one-necked, round-bottom flask, equipped with a magnetic stir-bar, and an argon inlet with a septum were placed ester 36 (57.9 mg, $0.138 \mathrm{mmol}, 1.0$ equiv) and THF (1 
$\mathrm{mL}$ ). To a 5-mL, one-necked, conical-flask, sealed with a septum was added potassium trimethylsilanoate (177 mg, $1.38 \mathrm{mmol}, 10$ equiv) along with THF $(1.5 \mathrm{~mL})$. The solution of TMSOK was transferred via cannula into the solution of $\mathbf{3 6}$ using THF $(0.5 \mathrm{~mL})$ to rinse the flask. The reaction solution turned orange/yellow upon addition of TMSOK. The solution was stirred for $4 \mathrm{~h}$ at room temperature, then the reaction was quenched with an aqueous solution of citric acid $(0.5 \mathrm{M}, 2 \mathrm{~mL})$ and the mixture was stirred for $10 \mathrm{~min}$. The solution turned brightyellow upon addition of citric acid. The mixture was transferred to $125-\mathrm{mL}$ separatory funnel containing water $(25 \mathrm{~mL})$ and the aqueous layer was extracted with $\mathrm{Et}_{2} \mathrm{O}(3 \times 15 \mathrm{~mL})$, and the combined organic layers were washed with brine $(10 \mathrm{~mL})$ and concentrated to a yellow oil. The crude product was purified by column chromatography (( $\left.\mathrm{SiO}_{2}, 40 \times 50 \mathrm{~mm}\right)$, hexane/EtOAc, 1/1) to afford $56 \mathrm{mg}(99 \%)$ of $\mathbf{2}$ as a yellow film.

Data for 2:

${ }^{1}$ H NMR: $\quad\left(500 \mathrm{MHz}, \mathrm{CDCl}_{3}\right)$

7.32 (dd, $J=15.8,10.5 \mathrm{~Hz}, 1 \mathrm{H}, \mathrm{CH}(3))$; 6.25-6.18 (m, 2H, $\mathrm{CH}(10)$ and $\mathrm{CH}(4))$; $6.12(\mathrm{dt}, J=15.3,7.5 \mathrm{~Hz}, 1 \mathrm{H}, \mathrm{CH}(5)) ; 5.92$ (d, $J=10.5 \mathrm{~Hz}, 1 \mathrm{H}, \mathrm{CH}(9)) ; 5.78$ (d, $J=15.8 \mathrm{~Hz}, 1 \mathrm{H}, \mathrm{CH}(2)) ; 5.66(\mathrm{dt}, J=14.5,7.2 \mathrm{~Hz}, 1 \mathrm{H}, \mathrm{CH}(11)) ; 4.06(\mathrm{t}, J=6.4$ $\mathrm{Hz}, 1 \mathrm{H}, \mathrm{CH}(7)) ; 2.46-2.30$ (m, 2H, CH(6)); 2.24-2.00 (m, 2H, CH(12)); 1.88 (s, $3 \mathrm{H}, \mathrm{CH}(18))$; $1.48-1.08\left(\mathrm{~m}, 5 \mathrm{H}, \mathrm{CH}_{2}(13)\right.$ and $\mathrm{CH}_{2}(15)$ and $\left.\mathrm{CH}(14)\right) ; 0.92(\mathrm{t}, J=$ $7.9 \mathrm{~Hz}, 9 \mathrm{H}, \mathrm{CH}(20)) ; 0.86(\mathrm{~d}, J=6.8 \mathrm{~Hz}, 3 \mathrm{H}, \mathrm{CH}(17)) ; 0.86$ (t, $J=7.2 \mathrm{~Hz}, 3 \mathrm{H}$, $\left.\mathrm{CH}_{3}(16)\right) ; 0.55$ (q, $\left.J=7.9 \mathrm{~Hz}, 6 \mathrm{H}, \mathrm{CH}(19)\right)$

${ }^{13} \mathrm{C} \mathrm{NMR}: \quad\left(126 \mathrm{MHz}, \mathrm{CDCl}_{3}\right)$

$172.21(\mathrm{C}(1)) ; 147.26(\mathrm{C}(3)) ; 142.47(\mathrm{C}(5)) ; 136.59(\mathrm{C}(8)) ; 135.30(\mathrm{C}(11))$; $129.91(\mathrm{C}(4)) ; 125.58(\mathrm{C}(10)) ; 125.56(\mathrm{C}(9)) ; 118.54(\mathrm{C}(2)) ; 77.37$ (C(7)); 40.49 (C(6)); 36.20 (C(13)); 34.04 (C(14)); 30.60 (C(12)); 29.36 (C(15)); 19.08 (C(17)); $11.90(\mathrm{C}(18)) ; 11.33(\mathrm{C}(16)) ; 6.82(\mathrm{C}(19)) ; 4.78(\mathrm{C}(20))$

IR: (film)

3025 (m); 2958 (s); 2914 (s); 2876 (s); 2360 (w); 2342 (w); 1689 (s); 1639 (m);

1616 (m); 1458 (w); 1417 (m); 1378 (w); 1303 (w); 1272 (m); 1241 (w); 1149 (w); 1067 (m); $1002(\mathrm{~s}) ; 965(\mathrm{~m}) ; 742(\mathrm{~m})$

MS: $\quad(\mathrm{ESI})$

$429\left(\left(\mathrm{M}^{+}+\mathrm{Na}(32)\right) ; 360(28) ; 338(100) ; 275\right.$ (71); 261(25) 
Opt. Rot.: $\quad[\alpha]_{\mathrm{D}}^{24} 6.90\left(\mathrm{c}=0.63, \mathrm{CHCl}_{3}\right)$

TLC: $\quad R_{f} 0.29$ (hexanes/EtOAc, 7/1) $\left[\mathrm{SiO}_{2}, \mathrm{CAM}\right]$

HRMS: $\quad \mathrm{C}_{24} \mathrm{H}_{42} \mathrm{O}_{3} \mathrm{SiNa}$

Calcd: $\quad 429.2825$

Found: $\quad 429.2821$

\section{Acylation and Global Deprotection}

Preparation of 1,1-Anhydro-4,6-O-di-tert-butylsilylene-3-O-(7' $S, 14$ ' $S)-2$ " $E, 4$ ' $E, 8^{\prime \prime} E, 10$ ' $E$ 8",14"'-dimethyl-7"'-triethylsilioxy-2",4",8", 10"-hexadecatetraenoyl)-2-O-(2-trimethylsilylethoxycarbonyl)-1-(2',5'-dihydroxy-6'-hydroxymethylphenyl)- $\alpha$ - $D$-glucopyranose [CSR-IX-58]<smiles>C/C(=C\C=C/CCC(C)C)C([O-])C/C=C/C=C/C(=O)O</smiles>

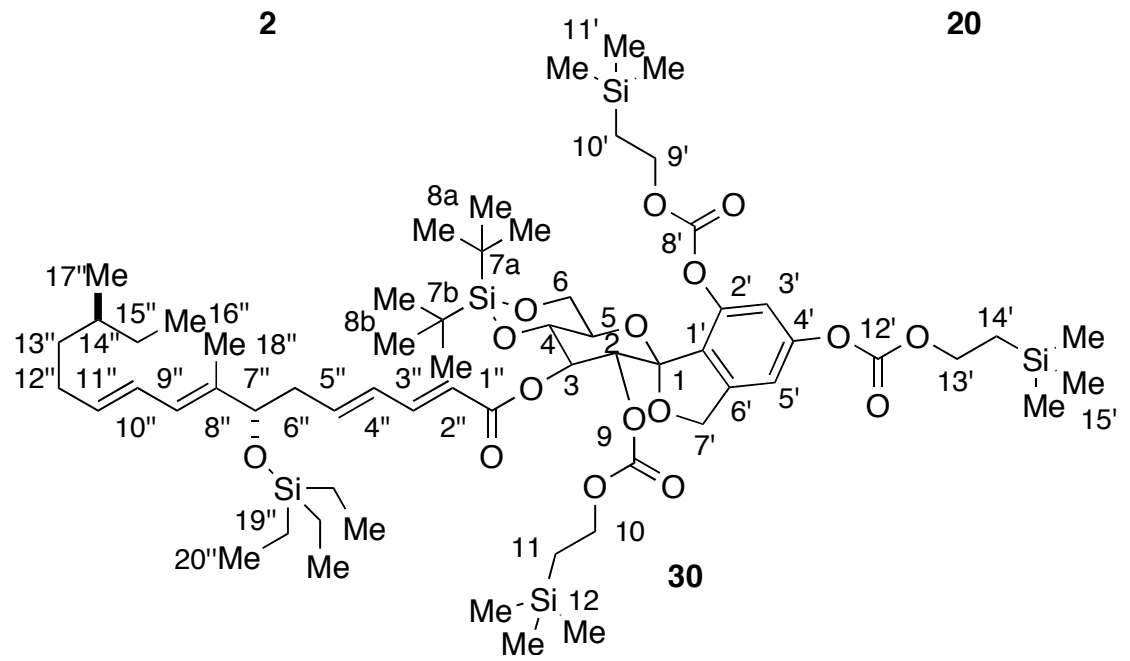

To a 10-mL Schlenk flask, flushed with argon, equipped with a magnetic stir-bar and a rubber septum was added acid 2 ( $0.048 \mathrm{~g}, 0.119 \mathrm{mmol}, 1.3$ equiv) followed by toluene $(1.7 \mathrm{~mL})$ and triethylamine $(141 \mu \mathrm{L}, 1.01 \mathrm{mmol}, 11$ equiv). To this yellow solution was added 2,4,6trichlorobenzoyl chloride ( $40 \mu \mathrm{L}, 0.258 \mathrm{~mol}, 2.8$ equiv) and the contents were stirred at room temperature for $1 \mathrm{~h}$. To a 5-mL, conical-flask, equipped with a magnetic stir-bar and an argon inlet with septum was added 20 (0.080 g, 0.092 mmol, 1.0 equiv) along with DMAP (0.029 g, $0.239 \mathrm{mmol}, 2.6$ equiv). Then toluene $(1.3 \mathrm{~mL})$ was added and the contents were stirred until all 
the DMAP had dissolved ( $\sim 5 \mathrm{~min})$. The solution of $\mathbf{2 0}$ and DMAP was cannula transferred into the Schlenk flask, washing the conical-flask with toluene $(0.2 \mathrm{~mL})$. The contents became heterogeneous upon the addition of DMAP and 20. This mixture was stirred at room temperature for $3 \mathrm{~h}$, then toluene $(7 \mathrm{~mL})$ was added, followed by saturated, aqueous $\mathrm{NaHCO}_{3}$ solution $(5 \mathrm{~mL})$. The contents of the flask were transferred to a $60-\mathrm{mL}$ separatory funnel and the aqueous layer was extracted. The aqueous extract was diluted with $\mathrm{H}_{2} \mathrm{O}(10 \mathrm{~mL})$ and extracted with toluene $(3 \times 17 \mathrm{~mL})$. The combined organic extracts were washed with $\mathrm{H}_{2} \mathrm{O}(1 \times 20 \mathrm{~mL})$, brine $(1 \times 20 \mathrm{~mL})$ and then were dried $\left(\mathrm{Na}_{2} \mathrm{SO}_{4}\right)$, filtered, and concentrated to a yellow oil. The crude product was purified using column chromatography $\left(\left(\mathrm{SiO}_{2}, 40 \times 180\right)\right.$, hexanes/EtOAc, 15:1 to $7: 1))$ to afford a viscous, colorless oil. The oil was dried under reduced pressure $(0.08$ $\mathrm{mmHg}$ ) at $40{ }^{\circ} \mathrm{C}$ for $8 \mathrm{~h}$ to afford $0.101 \mathrm{~g}(87 \%)$ of $\mathbf{3 0}$, as a colorless glass.

\section{Data for 30:}

${ }^{1}$ H NMR: $\quad\left(500 \mathrm{MHz}, \mathrm{CDCl}_{3}\right)$

$7.28\left(\mathrm{dd}, J=14.9,11.3 \mathrm{~Hz}, 1 \mathrm{H}, \mathrm{CH}\left(3^{\prime \prime}\right)\right) ; 7.11\left(\mathrm{~d}, J=1.8 \mathrm{~Hz}, 1 \mathrm{H}, \mathrm{CH}\left(3^{\prime}\right.\right.$ or $\left.\left.5^{\prime}\right)\right)$; $6.97\left(\mathrm{~d}, J=1.8 \mathrm{~Hz}, 1 \mathrm{H}, \mathrm{CH}\left(3^{\prime}\right.\right.$ or $\left.\left.5^{\prime}\right)\right)$; 6.24-6.14 (m, $2 \mathrm{H}, \mathrm{CH}\left(4^{\prime \prime}\right)$ and $\left.\mathrm{CH}\left(10^{\prime \prime}\right)\right)$; 6.07 (dt, $\left.J=15.7,7.8 \mathrm{~Hz}, 1 \mathrm{H}, \mathrm{CH}\left(5^{\prime \prime}\right)\right) ; 5.92\left(\mathrm{~d}, J=11.3 \mathrm{~Hz}, 1 \mathrm{H}, \mathrm{CH}\left(9^{\prime \prime}\right)\right) ; 5.82$ (d, $\left.J=14.9 \mathrm{~Hz}, 1 \mathrm{H}, \mathrm{CH}\left(2^{\prime \prime}\right)\right) ; 5.65(\mathrm{dt}, J=14.4,7.2 \mathrm{~Hz}, 1 \mathrm{H}, \mathrm{CH}(11 ")) ; 5.61$ (t, $J=$ $9.9 \mathrm{~Hz}, 1 \mathrm{H}, \mathrm{HC}(3)) ; 5.52$ (d, $J=9.9 \mathrm{~Hz}, 1 \mathrm{H}, \mathrm{HC}(2)) ; 5.20$ (s, $\left.2 \mathrm{H}, \mathrm{CH}_{2}\left(7^{\prime}\right)\right)$; $4.42-$ 4.29 (m, 4H, $\mathrm{CH}_{2}\left(9^{\prime}\right)$ and $\left.\mathrm{CH}_{2}\left(13^{\prime}\right)\right)$; 4.16-4.09 (m, 3H, $\mathrm{CH}(6 \mathrm{e})$ and $\left.\mathrm{CH}(10)\right)$; 4.07-3.90 (m, 4H, $\mathrm{CH}_{2}(10)$ and $\mathrm{CH}(6 \mathrm{a})$ and $\left.\mathrm{CH}(4)\right)$; 3.84-3.76 (m, 2H, $\mathrm{CH}(7 ")$ and $\mathrm{CH}(5))$; 2.44-2.27 (m, 2H, $\left.\mathrm{CH}_{2}(12 ")\right)$; 2.22-2.00 (m, 2H, $\mathrm{CH}_{2}\left(6^{\prime \prime}\right)$ ); 1.69 (s, $3 \mathrm{H}, \mathrm{CH}_{3}\left(18^{\prime \prime}\right)$ ); $1.46-1.30\left(\mathrm{~m}, 3 \mathrm{H}, \mathrm{CH}(14 ")\right.$ and $\mathrm{CH}_{2}\left(15^{\prime \prime}\right)$ ); 1.24-1.17 (m, $2 \mathrm{H}$, $\mathrm{CH}_{2}\left(13^{\prime \prime}\right)$ ); 1.18-1.09 (m, 4H, $\mathrm{CH}_{2}\left(1^{\prime}\right)$ and $\mathrm{CH}_{2}\left(14^{\prime}\right)$ ); 1.02 (s, $9 \mathrm{H}, \mathrm{C}\left(\mathrm{CH}_{3}\right)_{3}(8 \mathrm{a}$ or 8b)); 0.99 (s, 9H, C( $\left.\mathrm{CH}_{3}\right)_{3}(8 \mathrm{a}$ or $8 \mathrm{~b})$ ); 0.91 (t, $\left.J=7.8 \mathrm{~Hz}, 9 \mathrm{H}, \mathrm{CH}_{3}(20 ")\right) ; 0.87$ (d, $\left.J=6.1 \mathrm{~Hz}, 3 \mathrm{H}, \mathrm{CH}_{3}\left(17^{\prime \prime}\right)\right) ; 0.87$ (t, $\left.J=7.3 \mathrm{~Hz}, 3 \mathrm{H}, \mathrm{CH}_{3}(16 ")\right)$; 0.81-0.76 (m, 2H, $\left.\mathrm{CH}_{2}(11)\right) ; 0.54\left(\mathrm{q}, J=7.8 \mathrm{~Hz}, 6 \mathrm{H}, \mathrm{CH}_{2}\left(19^{\prime \prime}\right)\right) ; 0.09$ (s, 9H, $\mathrm{CH}_{3}\left(11^{\prime}\right)$ or $\left.\left(15^{\prime}\right)\right) ; 0.07$ (s, $9 \mathrm{H}, \mathrm{CH}_{3}\left(11^{\prime}\right)$ or $\left.\left(15^{\prime}\right)\right) ;-0.04\left(\mathrm{~s}, 9 \mathrm{H}, \mathrm{CH}_{3}(12)\right)$

\section{${ }^{13} \mathrm{C} \mathrm{NMR}: \quad\left(125 \mathrm{MHz}, \mathrm{CDCl}_{3}\right)$}

$\left.\left.166.04\left(\mathrm{C}\left(1^{\prime \prime}\right)\right) ; 154.13(9)\right) ; 153.23\left(2^{\prime}\right)\right) ; 152.88\left(\mathrm{C}\left(8^{\prime}\right.\right.$ or $\left.\left.12^{\prime}\right)\right) ; 152.46\left(\mathrm{C}\left(8^{\prime}\right.\right.$ or 12')); $146.72\left(\mathrm{C}\left(4^{\prime}\right)\right) ; 145.24\left(\mathrm{C}\left(3^{\prime \prime}\right)\right) ; 143.40\left(\mathrm{C}\left(1^{\prime}\right)\right) ; 140.99\left(\mathrm{C}\left(5^{\prime \prime}\right)\right) ; 136.74$ $\left.\left(\mathrm{C}\left(8^{\prime \prime}\right)\right) ; 135.21\left(\mathrm{C}\left(11^{\prime \prime}\right)\right) ; 130.17\left(4^{\prime \prime}\right)\right) ; 125.59\left(\mathrm{C}\left(10^{\prime \prime}\right)\right) ; 125.47\left(\mathrm{C}\left(9^{\prime \prime}\right)\right) ; 124.51$ 
$\left(\mathrm{C}\left(6^{\prime}\right)\right) ; 119.15\left(\mathrm{C}\left(2^{\prime \prime}\right)\right) ; 115.27\left(\mathrm{C}\left(3^{\prime}\right)\right) ; 111.59\left(\mathrm{C}\left(5^{\prime}\right)\right) ; 108.73(\mathrm{C}(1)) ; 77.45$ (C(7")); $77.20(\mathrm{C}(4)) ; 75.53(\mathrm{C}(2)) ; 74.53(\mathrm{C}(3)) ; 73.39$ (C(5)); 72.62 (C(9' or $\left.\left.13^{\prime}\right)\right) ; 68.92\left(\mathrm{C}\left(9^{\prime}\right.\right.$ or $\left.\left.1^{\prime}\right)\right) ; 67.72\left(\mathrm{C}\left(7^{\prime}\right)\right) ; 66.89(\mathrm{C}(6)) ; 66.61(\mathrm{C}(10)) ; 40.51$ (C(6")); 36.19 (C(13")); $34.04(\mathrm{C}(14 ")) ; 30.58\left(\mathrm{C}\left(12^{\prime \prime}\right)\right) ; 29.33$ (C(15")); 27.34 (C(8a or $8 b)) ; 26.87$ (C(8a or 8b)); 22.63 (C(7a or 7b)); 19.97 (C(7a or 7b)); 17.55 $\left(10^{\prime}\right.$ or $\left.\left.14^{\prime}\right)\right)$; $17.49\left(10^{\prime}\right.$ or $\left.\left.14^{\prime}\right)\right)$; $17.06\left(\mathrm{C}\left(13^{\prime \prime}\right)\right) ; 11.18\left(\mathrm{C}\left(18^{\prime \prime}\right)\right)$; $11.32\left(\mathrm{C}\left(16^{\prime \prime}\right)\right)$; $6.80\left(\mathrm{C}\left(19^{\prime \prime}\right)\right) ; 4.75\left(\mathrm{C}\left(20^{\prime \prime}\right)\right) ;-1.50\left(\mathrm{C}\left(11^{\prime}\right.\right.$ or $\left.\left.15^{\prime}\right)\right) ;-1.57\left(\mathrm{C}\left(11^{\prime}\right.\right.$ or $\left.\left.15^{\prime}\right)\right) ;-1.66$ $(\mathrm{C}(12))$

IR: (film)

2957 (s); 2933 (s); 2876 (s); 1764 (s); 1641 (w); 1459 (w); 1382 (w); 1250 (s); 1176 (m); 1096 (m); 1065 (m); 1012 (m); 973 (m); 859 (m); 873 (s)

MS: $\quad(\mathrm{ESI})$ 1283((M+ $+\mathrm{Na}(100)) ; 1019(13) ; 782(5) ; 489(18)$

Opt. Rot.: $\quad[\alpha]_{\mathrm{D}}^{24}-21.6\left(\mathrm{c}=0.51, \mathrm{CHCl}_{3}\right)$

TLC: $\quad R_{f} 0.12$ (hexanes/EtOAc, 10/1) $\left[\mathrm{SiO}_{2}, \mathrm{CAM}\right]$

HRMS: $\quad \mathrm{C}_{63} \mathrm{H}_{108} \mathrm{O}_{16} \mathrm{Si}_{5} \mathrm{Na}$

Calcd: $\quad 1283.6382$

Found: $\quad 1283.6431$

Preparation of 1,1-Anhydro-1,C[-6-hydroxymethyl)-2,4-phenyl]-3-O-[(7" $S, 14 " S)-8$ "-14--dimethyl-7"-(hydroxy)hexadecane-2" $E, 4 " E, 8^{\prime \prime} E, 10 " E$-tetraenoyl]- $\alpha$-D-glucopyranose or Papulacandin D [CSR-IX-95]
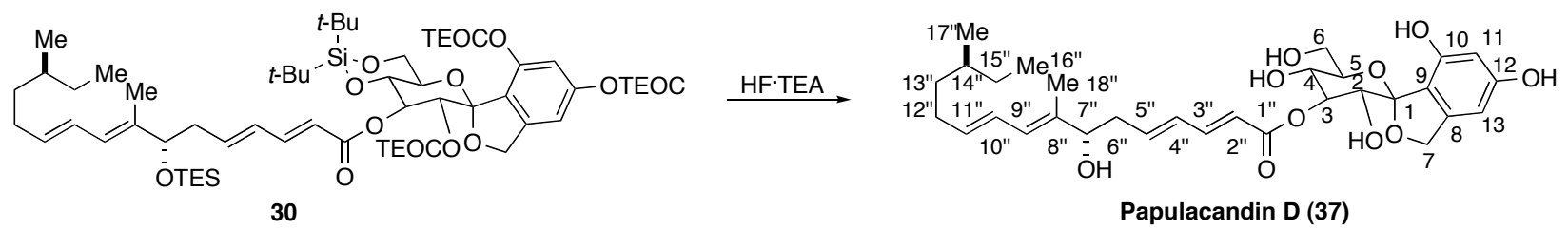

To a $25-\mathrm{mL}$, Teflon-flask, equipped with a magnetic stir-bar, an air condenser, and an argon inlet with a rubber septum) was added protected papulacandin D (30) (0.045 g, 0.037 mmol, 1.0 equiv and the apparatus was flushed with argon. To a separate plastic container equipped with a magnetic stir bar was added DMSO $(10 \mathrm{~mL})$, triethylamine $(16.29 \mathrm{~mL})$, and HF 
$(49 \%, 2.5 \mathrm{~mL})$ and the contents were stirred vigorously. Then $6 \mathrm{~mL}$ of this buffered HF solution (14.26 mmol, 400 equiv, fluoride) was added to the Teflon flask via syringe. The contents were stirred at room temperature for $5 \mathrm{~min}$, then at $60{ }^{\circ} \mathrm{C}$ (oil bath temperature) for $18 \mathrm{~h}$. The biphasic yellow reaction mixture was directly subjected to column chromatography $\left(\left(\mathrm{SiO}_{2}, 50 \times 100 \mathrm{~mm}\right)\right.$, EtOAc to EtOAc/MeOH, 9/1)). DMSO was removed form the isolated product under reduced pressure $\left(8.5 \times 10^{-5} \mathrm{mmHg}\right)$ at $30{ }^{\circ} \mathrm{C}$ for $8 \mathrm{~h}$. Then the resulting, yellow film was once again purified using column chromatography (( $\left.\mathrm{SiO}_{2}, 20 \times 10 \mathrm{~mm}\right)$, chloroform/ $\left.\left.\mathrm{MeOH}, 9 / 1\right)\right)$. After concentration the contents were dissolved in EtOAc $(10 \mathrm{~mL})$, filtered and concentrated in vacuo to afford $22 \mathrm{mg}(89 \%)$ of papulacandin D as a light-yellow foam.

Data for Papulacandin D:

${ }^{1}$ H NMR: $\quad\left(500 \mathrm{MHz}, \mathrm{CD}_{3} \mathrm{OD}\right)$

$7.30(\mathrm{dd}, J=15.8,11.5 \mathrm{~Hz}, 1 \mathrm{H}, \mathrm{CH}(3 ")) ; 6.25$ (ddt, $J=15.7,10.8$, and $1.6 \mathrm{~Hz}$, $\left.1 \mathrm{H}, \mathrm{CH}\left(4^{\prime \prime}\right)\right) ; 6.23(\mathrm{dd}, J=14.9,10.7 \mathrm{~Hz}, 1 \mathrm{H}, \mathrm{CH}(10 ")) ; 6.20$ (m, 1H, CH(11)); $6.19(\mathrm{~m}, 1 \mathrm{H}, \mathrm{CH}(13)) ; 6.12(\mathrm{dt}, J=15,4,14.9 \mathrm{~Hz}, 1 \mathrm{H}, \mathrm{CH}(5 ")) ; 5.98$ (dd, $J=$ 10.7, $\left.0.6 \mathrm{~Hz}, 1 \mathrm{H}, \mathrm{CH}\left(9^{\prime \prime}\right)\right) ; 5.92$ (d, $\left.J=15.3 \mathrm{~Hz}, 1 \mathrm{H}, \mathrm{CH}(2 ")\right) ; 5.66$ (dt, $J=14.9$, $\left.7.3 \mathrm{~Hz}, 1 \mathrm{H}, \mathrm{CH}\left(11^{\prime \prime}\right)\right) ; 5.34$ (t, $\left.J=9.7 \mathrm{~Hz}, 1 \mathrm{H}, \mathrm{CH}(3)\right) ; 5.02$ (ABq, $J=12.9 \mathrm{~Hz}$, 2H, $\left.\mathrm{CH}_{2}(7)\right) ; 4.34$ (d, $\left.J=10.1 \mathrm{~Hz}, 1 \mathrm{H}, \mathrm{CH}(2)\right) ; 4.07$ (t, $J=6.6 \mathrm{~Hz}, 1 \mathrm{H}, \mathrm{CH}\left(7^{\prime \prime}\right)$ ); 3.88 (ddd, $J=10.3,4.8$, and $2.4 \mathrm{~Hz}, 1 \mathrm{H}, \mathrm{CH}(5)$ ); 3.79-3.66 (m, $\left.2 \mathrm{H}, \mathrm{CH}_{2}(6)\right), 3.68$ (t, $J=9.8 \mathrm{~Hz}, 1 \mathrm{H}, \mathrm{CH}(4)) ; 2.42\left(\mathrm{t}, J=7.2 \mathrm{~Hz}, 2 \mathrm{H}, \mathrm{CH}_{2}(6 ")\right) ; 2.18-2.04$ (m, $2 \mathrm{H}$, $\left.\mathrm{CH}_{2}(12 ")\right) ; 1.71\left(\mathrm{~s}, 3 \mathrm{H}, \mathrm{CH}_{3}\left(18^{\prime \prime}\right)\right) ; 1.50-1.30\left(\mathrm{~m}, 2 \mathrm{H}, \mathrm{CH}_{2}(13 ")\right)$; 1.30-1.11 (m, $\left.2 \mathrm{H}, \mathrm{CH}_{2}\left(15^{\prime \prime}\right)\right) ; 1.28-1.11$ (m, $\left.1 \mathrm{H}, \mathrm{CH}(14 ")\right) ; 0.87$ (t, $J=7.3 \mathrm{~Hz}, 3 \mathrm{H}, \mathrm{CH}_{3}\left(16^{\prime \prime}\right)$ ); $0.87\left(\mathrm{~d}, J=6.5 \mathrm{~Hz}, 3 \mathrm{H}, \mathrm{CH}_{3}\left(17^{\prime \prime}\right)\right)$

\section{${ }^{13} \mathrm{C}$ NMR: $\quad\left(125 \mathrm{MHz}, \mathrm{CDCl}_{3}\right)$}

$169.15(\mathrm{C}(1 ")) ; 161.74(\mathrm{C}(12)) ; 154.86(\mathrm{C}(10)) ; 146.63(\mathrm{C}(3 ")) ; 145.73(\mathrm{C}(8))$; $\left.142.06\left(\mathrm{C}\left(5^{\prime \prime}\right)\right) ; 137.70\left(\mathrm{C}\left(8^{\prime \prime}\right)\right) ; 136.36(\mathrm{C}(11 ")) ; 131.67\left(\mathrm{C}\left(10^{\prime \prime}\right)\right) ; 127.27\left(4^{\prime \prime}\right)\right)$; $127.22\left(\mathrm{C}\left(9^{\prime \prime}\right)\right) ; 121.14\left(\mathrm{C}\left(2^{\prime \prime}\right)\right) ; 116.76(\mathrm{C}(9)) ; 112.22(\mathrm{C}(1)) ; 103.01(\mathrm{C}(11))$; 100.01 (13)); 78.46 (C(3)); 77.71 (C(7")); 75.94 (C(5)); 71.99 (C(2)); 69.87 (C(4)); 62.62 (C(6)); $40.09\left(\mathrm{C}\left(6^{\prime \prime}\right)\right) ; 37.68$ (C(13")); 35.36 (C(14")); 31.74 $\left(\mathrm{C}\left(12^{\prime \prime}\right)\right) ; 30.59\left(\mathrm{C}\left(15^{\prime \prime}\right)\right) ; 19.59\left(\mathrm{C}\left(18^{\prime \prime}\right)\right) ; 12.29\left(\mathrm{C}\left(17^{\prime \prime}\right)\right) ; 11.56\left(\mathrm{C}\left(16^{\prime \prime}\right)\right)$

IR: (film)

3351 (br, s); 2960 (s); 2855 (s); 1698 (s); 1640 (s); 1613 (s); 1463 (s); 1377 (s); 
1260 (s); 1006 (s); 978 (s); 885 (w)

MS: $\quad(E S I)$

575(( $\left.\mathrm{M}^{+}+1(75)\right) ; 359$ (100); 782 (5); 283 (13)

Opt. Rot.: $\quad[\alpha]_{\mathrm{D}}^{24} 8.78(\mathrm{c}=0.21, \mathrm{MeOH})$

TLC: $\quad R_{f} 0.46$ (chloroform/MeOH, 4/1) $\left[\mathrm{SiO}_{2}, \mathrm{UV}, \mathrm{I}_{2}\right]$

UV-Vis: $\quad \lambda_{\max }(232) ;(238) ;(260)$

HRMS: $\quad \mathrm{C}_{31} \mathrm{H}_{43} \mathrm{O}_{10}$

Calcd: $\quad 575.2856$

Found: $\quad 575.2874$ 
Table 2: ${ }^{13} \mathrm{C}$ NMR Spectroscopic Properties

\begin{tabular}{|c|c|c|c|c|}
\hline Carbon No. & Natural $(\mathrm{ppm})^{19}$ & CSR-IX-95 (ppm) & Synthetic $(\mathrm{ppm})^{18}$ & Difference (ppm) \\
\hline 1 & 111.9 & 112.22 & 112.1 & 0.12 \\
\hline 2 & 78.2 & 71.99 & 71.9 & 0.09 \\
\hline 3 & 75.5 & 78.46 & 78.4 & 0.06 \\
\hline 4 & 73.7 & 69.87 & 69.8 & 0.07 \\
\hline 5 & 71.7 & 75.94 & 75.8 & 0.14 \\
\hline 6 & 62.2 & 62.62 & 62.5 & 0.12 \\
\hline 7 & 69.5 & 73.95 & 73.8 & 0.15 \\
\hline 8 & 145.3 & 145.73 & 145.5 & 0.23 \\
\hline 9 & 116.4 & 116.76 & 116.7 & 0.06 \\
\hline 10 & 161.4 & 154.86 & 154.7 & 0.16 \\
\hline 11 & 100.0 & 103.01 & 103.0 & 0.01 \\
\hline 12 & 154.4 & 161.74 & 161.5 & 0.24 \\
\hline 13 & 103.0 & 100.10 & 99.9 & 0.2 \\
\hline $1 "$ & 169.0 & 169.15 & 169.0 & 0.15 \\
\hline $2^{\prime \prime}$ & 120.7 & 121.14 & 120.9 & 0.24 \\
\hline $3 "$ & 146.4 & 146.63 & 146.4 & 0.23 \\
\hline $4 "$ & 127.1 & 127.27 & 127.1 & 0.17 \\
\hline $5 "$ & 137.4 & 142.06 & 141.8 & 0.26 \\
\hline $6^{\prime \prime}$ & 39.8 & 40.09 & 40.0 & 0.09 \\
\hline $7 "$ & 77.3 & 77.71 & 77.5 & 0.21 \\
\hline $8^{\prime \prime}$ & 136.0 & 137.7 & 137.5 & 0.2 \\
\hline $9^{\prime \prime}$ & 131.3 & 127.22 & 127.0 & 0.22 \\
\hline $10^{\prime \prime}$ & 126.9 & 131.67 & 131.5 & 0.17 \\
\hline $11^{\prime \prime}$ & 141.8 & 136.36 & 136.2 & 0.16 \\
\hline $12^{\prime \prime}$ & 31.4 & 31.74 & 31.6 & 0.14 \\
\hline $13^{\prime \prime}$ & 37.3 & 37.68 & 37.5 & 0.18 \\
\hline $14 "$ & 35.0 & 35.36 & 35.2 & 0.16 \\
\hline $15^{\prime \prime}$ & 30.3 & 30.59 & 30.4 & 0.19 \\
\hline $16^{\prime \prime}$ & 11.6 & 11.86 & 11.7 & 0.16 \\
\hline $17 "$ & 19.4 & 12.29 & 12.2 & 0.09 \\
\hline $18^{\prime \prime}$ & 12.2 & 19.59 & 19.4 & 0.19 \\
\hline
\end{tabular}


Table 3: ${ }^{1} \mathrm{H}$ NMR Spectroscopic Properties

\begin{tabular}{|c|c|c|c|}
\hline $\begin{array}{l}\text { Hydrogen } \\
\text { No. }\end{array}$ & Natural (ppm) ${ }^{19}$ & CSR-IX-95 (ppm) & Synthetic (ppm) ${ }^{18}$ \\
\hline 2 & $4.34(\mathrm{~d}, J=10 \mathrm{~Hz})$ & $4.34(\mathrm{~d}, J=10.1 \mathrm{~Hz})$ & $4.33(\mathrm{~d}, J=10.0 \mathrm{~Hz})$ \\
\hline 3 & $5.34(\mathrm{t}, J=10 \mathrm{~Hz})$ & $5.34(\mathrm{t}, J=9.7 \mathrm{~Hz})$ & $5.34(\mathrm{t}, J=9.7 \mathrm{~Hz})$ \\
\hline 4 & - & $3.68(\mathrm{t}, J=9.8 \mathrm{~Hz})$ & $3.68(\mathrm{t}, 9.7 \mathrm{~Hz})$ \\
\hline 5 & - & $\begin{array}{c}3.88(\mathrm{ddd}, J=10.3,4.8 \\
\text { and } 2.4 \mathrm{~Hz})\end{array}$ & $\begin{array}{c}3.87(\mathrm{ddd}, J=10.1 \\
4.8, \text { and } 2.3 \mathrm{~Hz})\end{array}$ \\
\hline 6 & - & $3.79-3.66(\mathrm{~m})$ & $3.70-3.66(\mathrm{~m})$ \\
\hline 7 & $5.03(\mathrm{ABq}, J=12 \mathrm{~Hz})$ & $\begin{array}{c}5.02(\mathrm{ABq}, J=12.9 \\
\mathrm{Hz})\end{array}$ & $\begin{array}{c}5.03(\mathrm{ABq}, J=12.6 \\
\mathrm{Hz})\end{array}$ \\
\hline 11 & $6.19(\mathrm{~s})$ & $6.20(\mathrm{~m})$ & $6.20(\mathrm{~m})$ \\
\hline 13 & $6.20(\mathrm{~s})$ & $6.19(\mathrm{~m})$ & $6.19(\mathrm{~m})$ \\
\hline $2^{\prime \prime}$ & - & $5.92(\mathrm{~d}, J=15.3 \mathrm{~Hz})$ & $5.92(\mathrm{~d}, J=15.3 \mathrm{~Hz})$ \\
\hline $3 "$ & - & $\begin{array}{c}7.30(\mathrm{dd}, J=15.8 \mathrm{~Hz} \\
11.5 \mathrm{~Hz})\end{array}$ & $\begin{array}{c}7.30(\mathrm{dd}, J=15.2 \\
10.1 \mathrm{~Hz})\end{array}$ \\
\hline $4 "$ & - & $\begin{array}{c}6.25(\mathrm{ddt}, J=15.7 \\
10.8, \text { and } 1.6 \mathrm{~Hz})\end{array}$ & $\begin{array}{c}6.25(\mathrm{ddt}, J=15.0 \\
10.8, \text { and } 1.3 \mathrm{~Hz})\end{array}$ \\
\hline $5^{\prime \prime}$ & - & $\begin{array}{c}6.12(\mathrm{dt}, J=15.4 \text { and } \\
14.9 \mathrm{~Hz})\end{array}$ & $\begin{array}{c}6.12(\mathrm{dt}, J=15.2 \\
14.7 \mathrm{~Hz})\end{array}$ \\
\hline $6 "$ & - & $2.42(\mathrm{t}, J=7.2 \mathrm{~Hz})$ & $2.42(\mathrm{t}, J=7.0 \mathrm{~Hz})$ \\
\hline $7 "$ & - & $4.07(\mathrm{t}, J=6.6 \mathrm{~Hz})$ & $4.07(\mathrm{t}, J=6.6 \mathrm{~Hz})$ \\
\hline 9" & - & $\begin{array}{c}5.98(\mathrm{dd}, J=10.7,0.6 \\
\mathrm{Hz})\end{array}$ & $\begin{array}{c}6.00(\mathrm{dd}, J=10.8 \\
0.7 \mathrm{~Hz})\end{array}$ \\
\hline $10^{\prime \prime}$ & - & $\begin{array}{c}6.23(\mathrm{dd}, J=14.9,10.7 \\
\mathrm{Hz})\end{array}$ & $\begin{array}{c}6.23(\mathrm{dd}, J=14.7 \\
10.7 \mathrm{~Hz})\end{array}$ \\
\hline $11 "$ & - & $\begin{array}{c}5.66(\mathrm{dt}, J=7.3 \mathrm{~Hz} \\
14.9 \mathrm{~Hz})\end{array}$ & $\begin{array}{c}5.66(\mathrm{dt}, J=7.0 \mathrm{~Hz} \\
15.0 \mathrm{~Hz})\end{array}$ \\
\hline $12^{\prime \prime}$ & - & $2.18-2.04(\mathrm{~m})$ & $2.18-2.04(\mathrm{~m})$ \\
\hline $13^{\prime \prime}$ & - & $1.50-1.30(\mathrm{~m})$ & $1.49-1.29(\mathrm{~m})$ \\
\hline $14 "$ & - & $1.28-1.11(\mathrm{~m})$ & $1.28-1.11(\mathrm{~m})$ \\
\hline $15^{\prime \prime}$ & - & $1.30-1.10(\mathrm{~m})$ & $1.30-1.10(\mathrm{~m})$ \\
\hline $16^{\prime \prime}$ & - & $0.87(\mathrm{t}, J=7.3 \mathrm{~Hz})$ & $0.87(\mathrm{t}, J=7.3 \mathrm{~Hz})$ \\
\hline $17 "$ & - & $0.87(\mathrm{~d}, J=6.5 \mathrm{~Hz})$ & $0.87(\mathrm{~d}, J=6.6 \mathrm{~Hz})$ \\
\hline $18^{\prime \prime}$ & - & $1.71(\mathrm{~s})$ & $1.71(\mathrm{~d}, J=0.5 \mathrm{~Hz})$ \\
\hline
\end{tabular}


Table 4: Physiochemical Properties

\begin{tabular}{ccc}
\hline Property & Natural $^{19}$ & CSR-IX-p95 \\
\hline Appearance & white foam & light yellow foam \\
m.p. & $127 \sim 130{ }^{\circ} \mathrm{C}$ & $126-128{ }^{\circ} \mathrm{C}$ \\
Spec. Opt. Rot. & $+7 \pm 1{ }^{\circ}(\mathrm{MeOH})$ & $8.78(\mathrm{c}=0.21, \mathrm{MeOH})$ \\
UV $\lambda_{\max (\mathrm{nm})}$ & $230,235,261(\mathrm{EtOH})$ & $232,238,260(\mathrm{EtOH})$ \\
& $0.58($ silica gel, & $0.46($ silica gel, \\
TLC & $\left.\mathrm{CHCl}_{3} / \mathrm{MeOH}, 4 / 1\right)$ & $\left.\mathrm{CHCl}_{3} / \mathrm{MeOH}, 4 / 1\right)$ \\
\hline
\end{tabular}




\section{References}

(1) Ukai, T.; Kawazura, H.; Ishii, Y.; Bonnett, J. J.; Ibers, J. A. J. Organomet. Chem. 1974, 253-266.

(2) Ishii, Y.; Hasegawa, S.; Kimura, S. Itoh, K. J. Organomet. Chem. 1974, 411-418.

(3) Hoye, T. R.; Aspass, A. W.; Eklov, B. M.; Ryba, T. D. Org. Lett. 2005, 7, 2205-2208.

(4) Gilman, H.; Cartledge, F. K.; Sim, S. Y. J. Organomet. Chem. 1963, 8-14.

(5) McDonald, R. N.; Steppel, R. N.; Dorsey, J. E. Org. Synth. Coll. Vol. 6, 276-277.

(6) Brown, H. C.; Kramer, G. W.; Levy, A. B.; Midland, M. M. Organic Synthesis via Boranes; Wiley: New York, 1973, 21-244.

(7) Krishnamurthy, S.; Brown, H. C.; J. Org. Chem. 1980, 45, 849-856.

(8) Yamazaki, N.; Dokoshi, W.; Kibayashi, C. Org. Lett. 2001, 3, 193-196.

(9) Takaya, H.; Ohta, T.; Inoue, S.-I.; Tokunaga, M.; Kitamura, M.; Noyori, R. Org. Synth. Coll. Vol. 9, 169-175.

(10) Kira, M.; Hino, T.; Sakurai, H. Tetrahedron Lett. 1989, 30, 1099-1102.

(11) (a) Denmark, S. E.; Fu, J. Lawler, M. J. Org. Synth. Vol. 83, 121-130. (b) Denmark, S. E.; Fu, J.; Coe, D. M.; Su, X.; Pratt, N.; Griedel, B. D. J. Org. Chem. 2006, 71, 1513-1522.

(12) Corey, E. J.; Hopkins, P. B. Tetrahedron Lett. 1982, 23, 4871-4874.

(13) Li, Y.; Hesse, M. Helv. Chim. Acta. 2003, 86, 310-323.

(14) Gioeli, C.; Balgobin, N.; Josephson, S.; Chattopadhyaya, J. B. Tetrahedron Lett. 1981, 22, 969-972.

(15) Roth.W.; Pigman, W. Methods Carbohydr. Chem. 1963, 2, 405-408.

(16) Aratani, N.; Takagi, A; Yanagawa, Y.; Matsumoto, T.; Kawai, T.; Yoon, Z. S.; Kim, D.; Osuka, A. Chem.-Eur. J. 2005, 11, 3389-3404.

(17) Boucard. V.; Larrieu. K.; Lubin-Germain, N.; Uziel, J; Augé, J. Synlett, 2003, 12, 18341837.

(18) Barrett, A. G. M.; Peña, M.; Willardsen, J.A. J. Org. Chem. 1996, 61, 1082-1100.

(19) (a) Traxler, P.; Gruner, J.; Auden, J. A. J. Antibiotics 1977, 30, 289-296. (b) Traxler, P.; Fritz, H.; Fuhrer, H.; Richter, W. J. Antibiotics 1980, 33, 967-978 
Denmark, Regens, Kobayashi

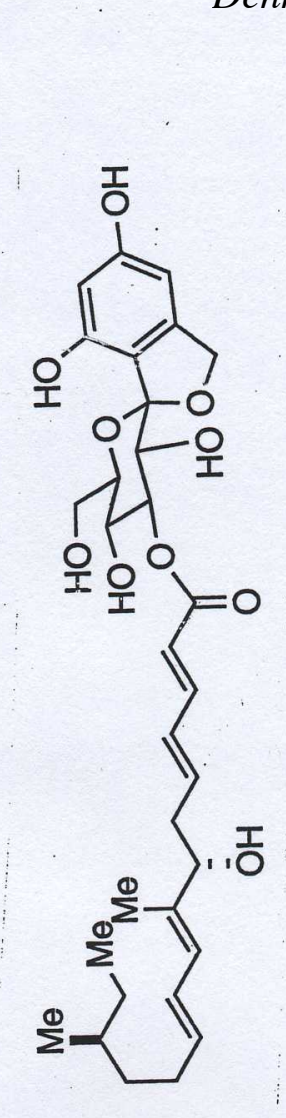

$$
\text { , Regens, Kobayashi }
$$
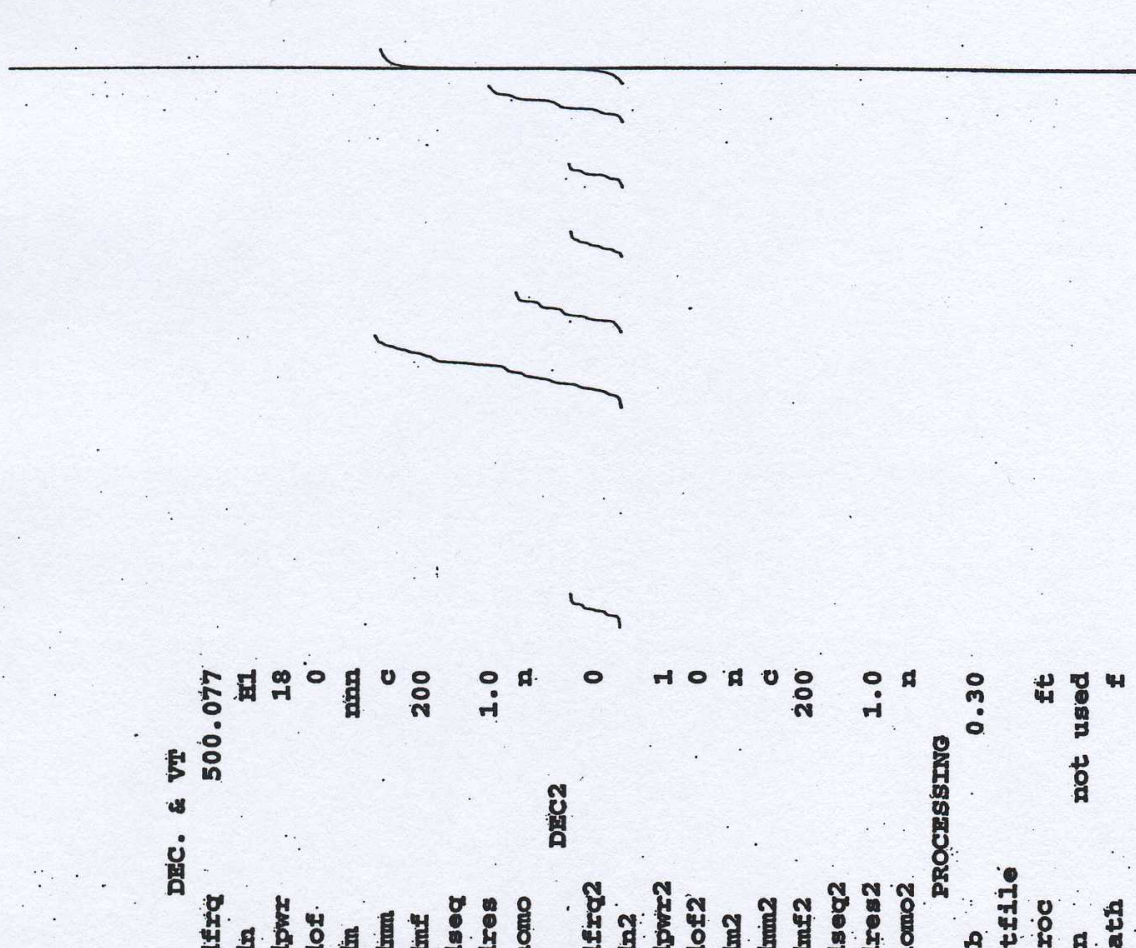

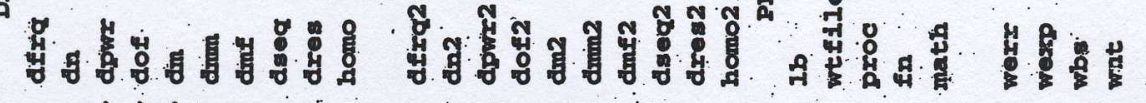

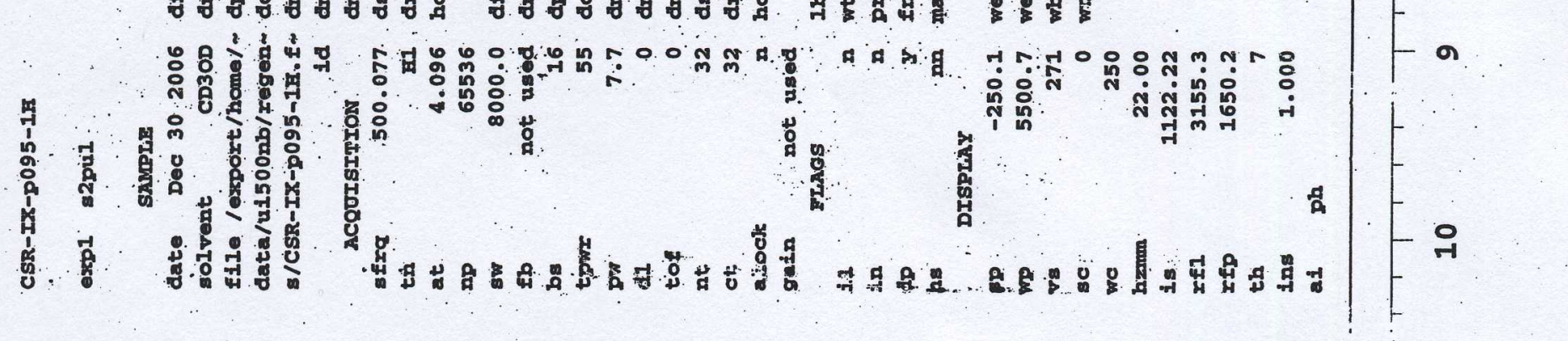




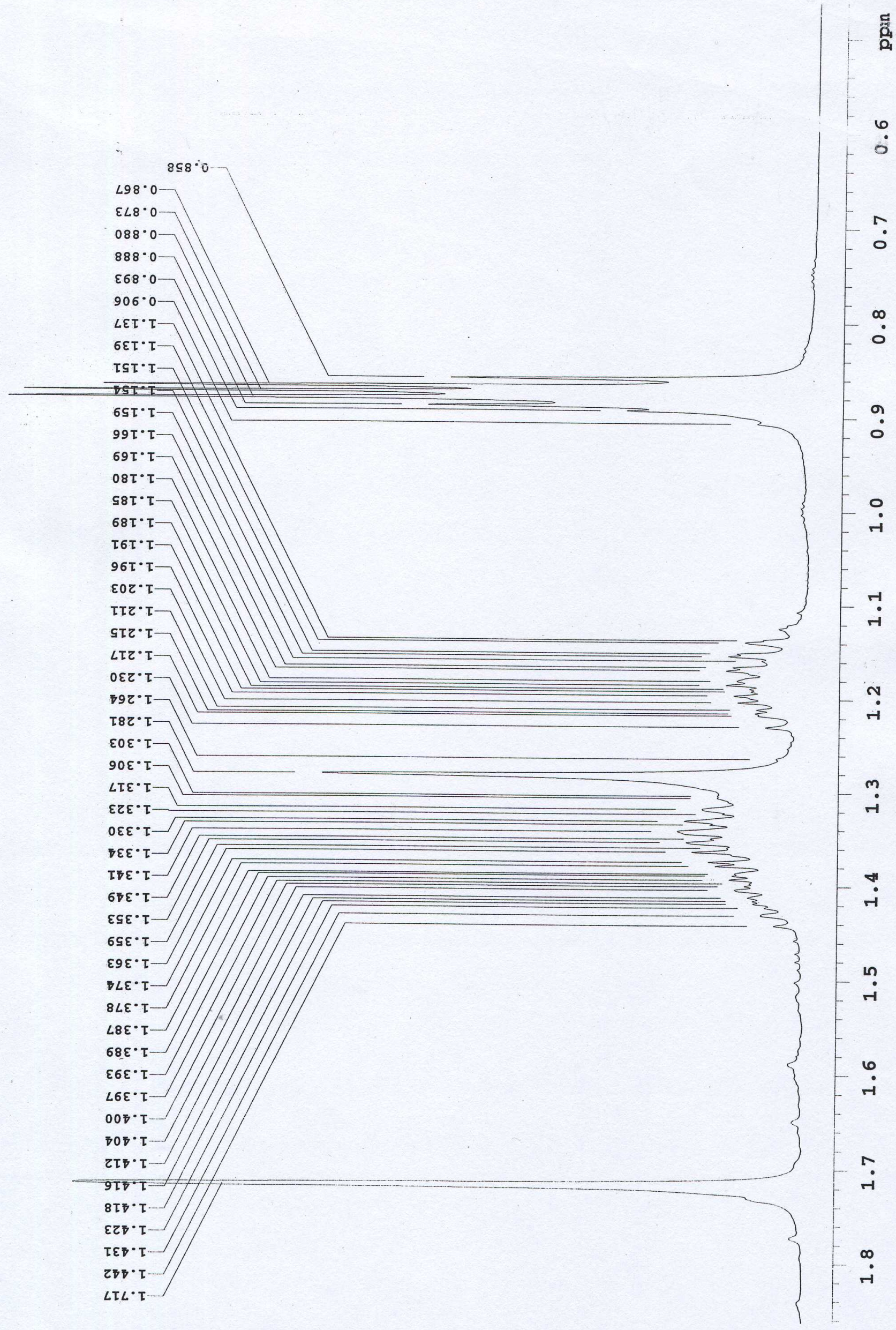


Denmark, Regens, Kobayashi

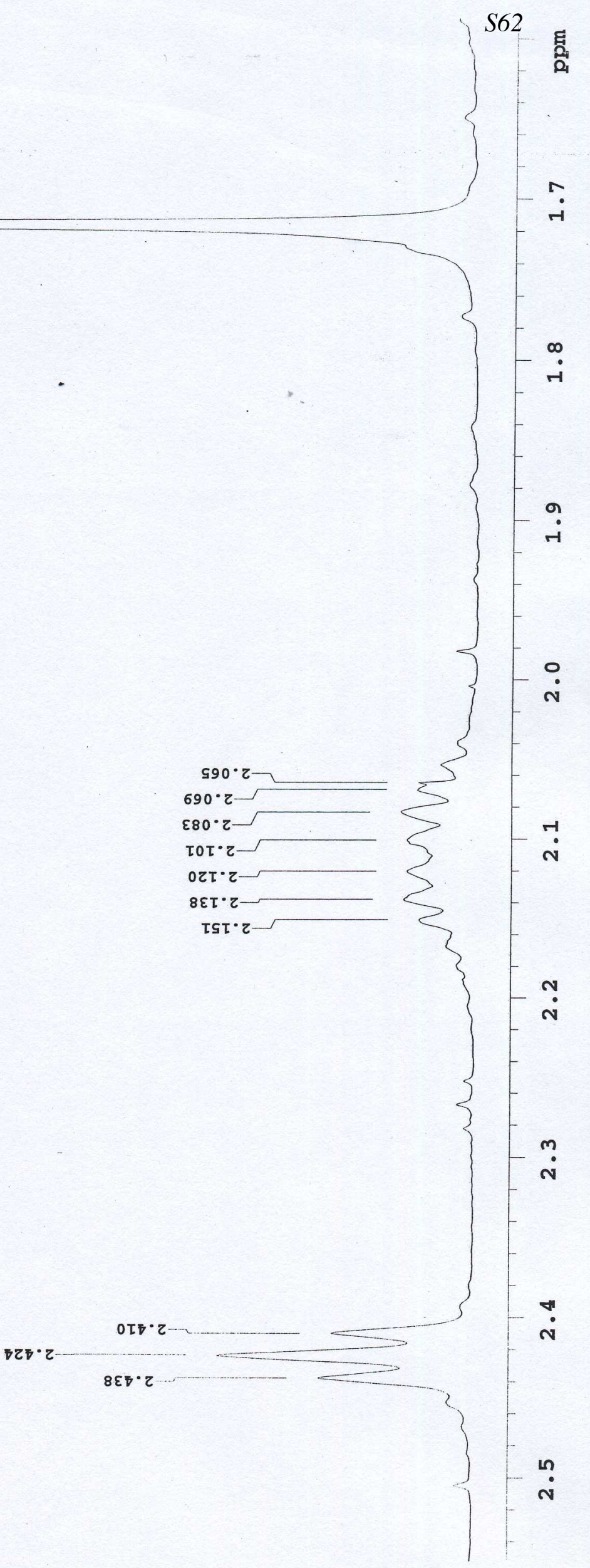




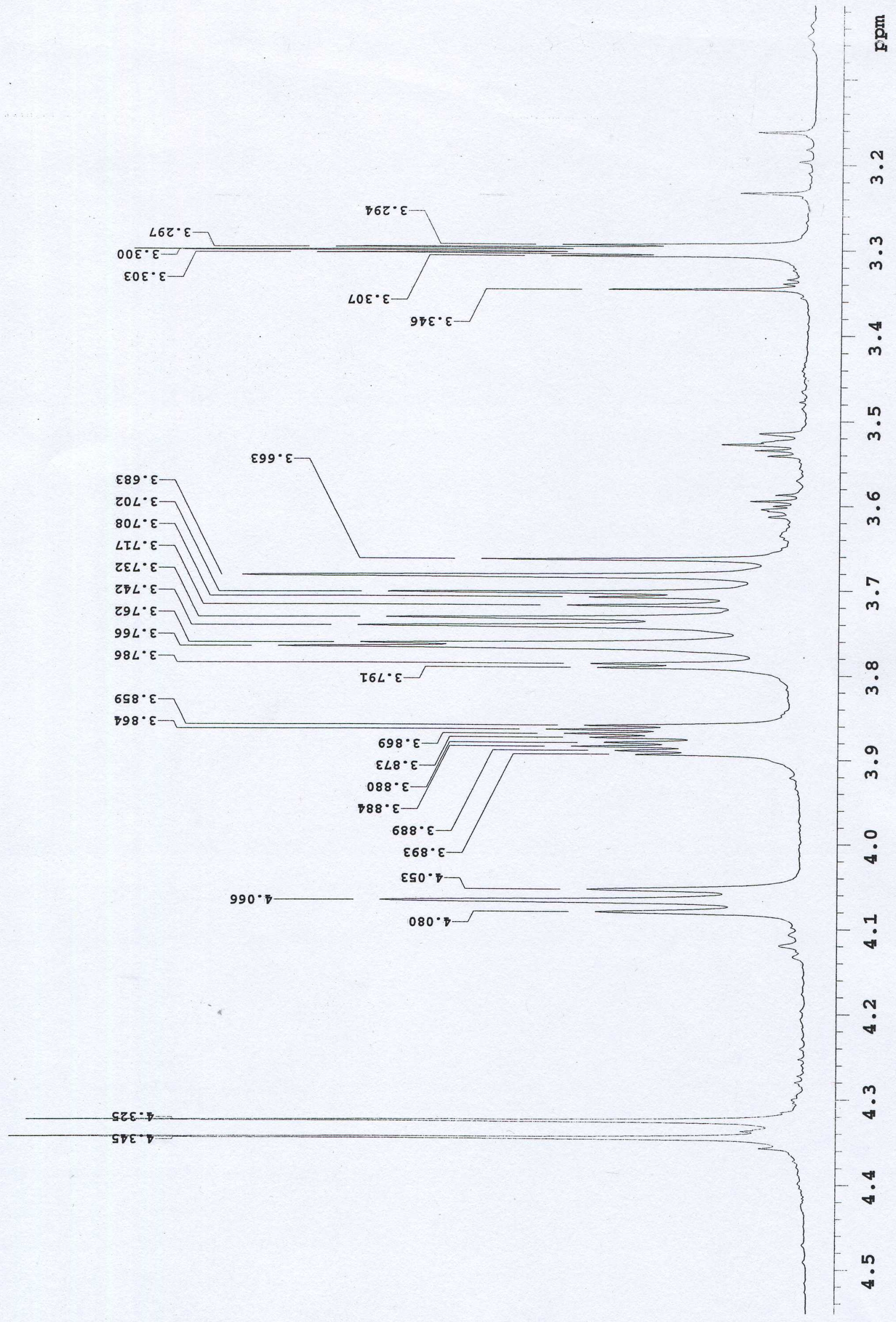


Denmark, Regens, Kobayashi

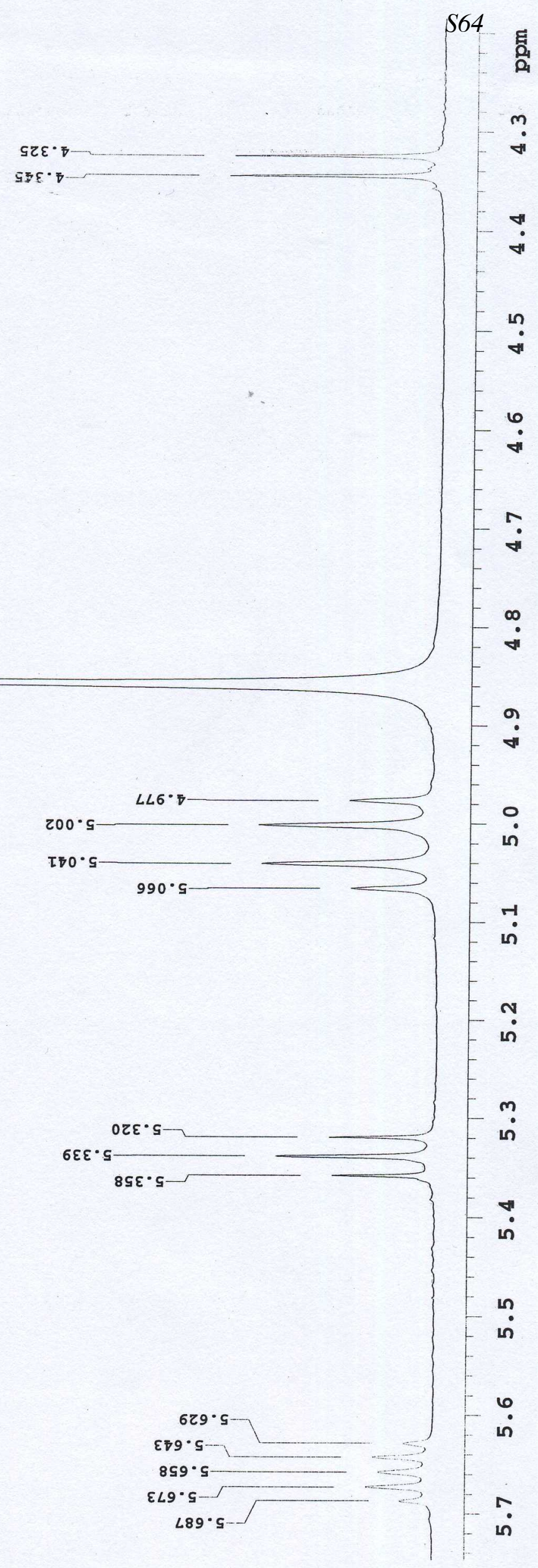


Denmark, Regens, Kobayashi

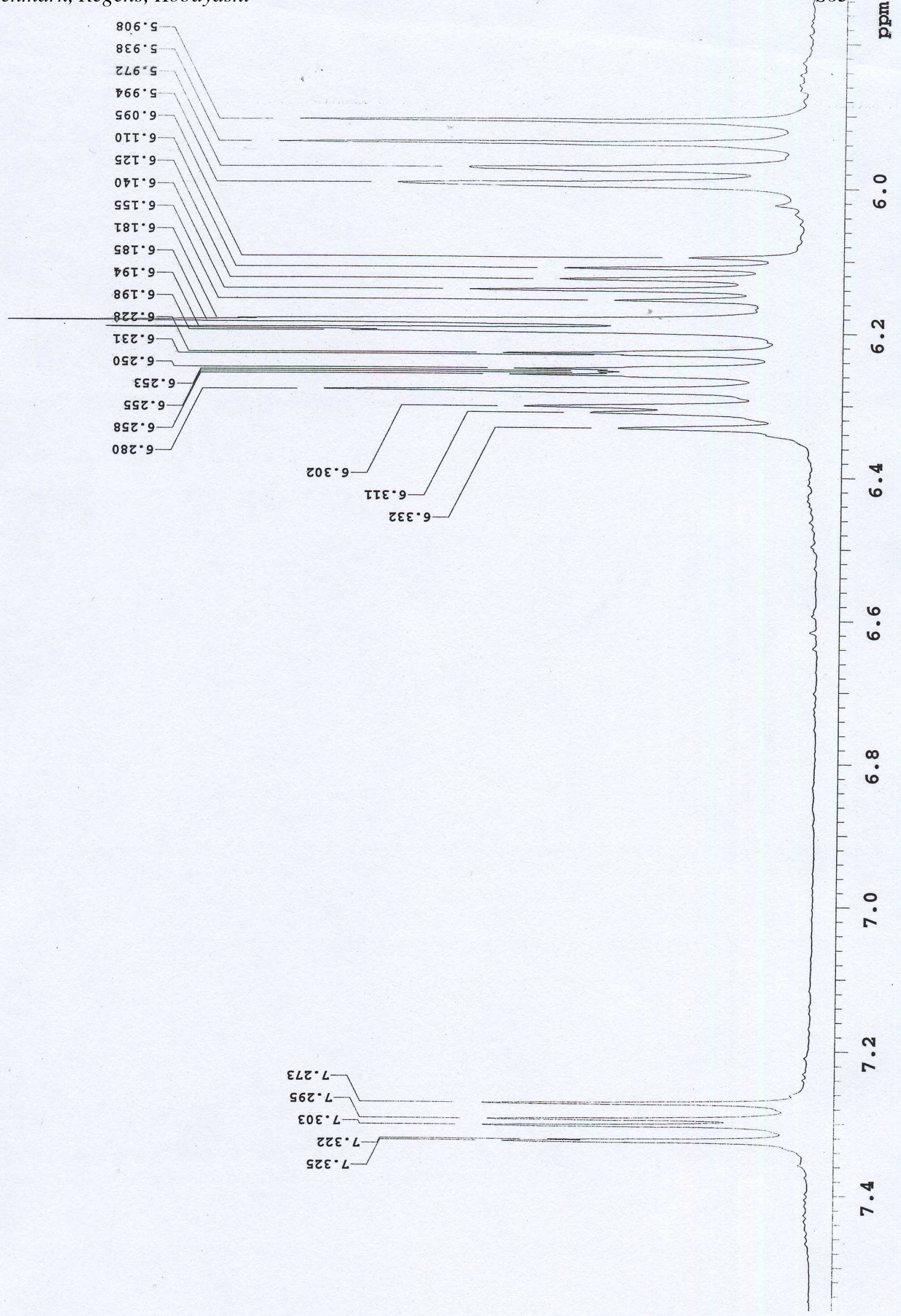


Denmark, Regens, Kobayashi
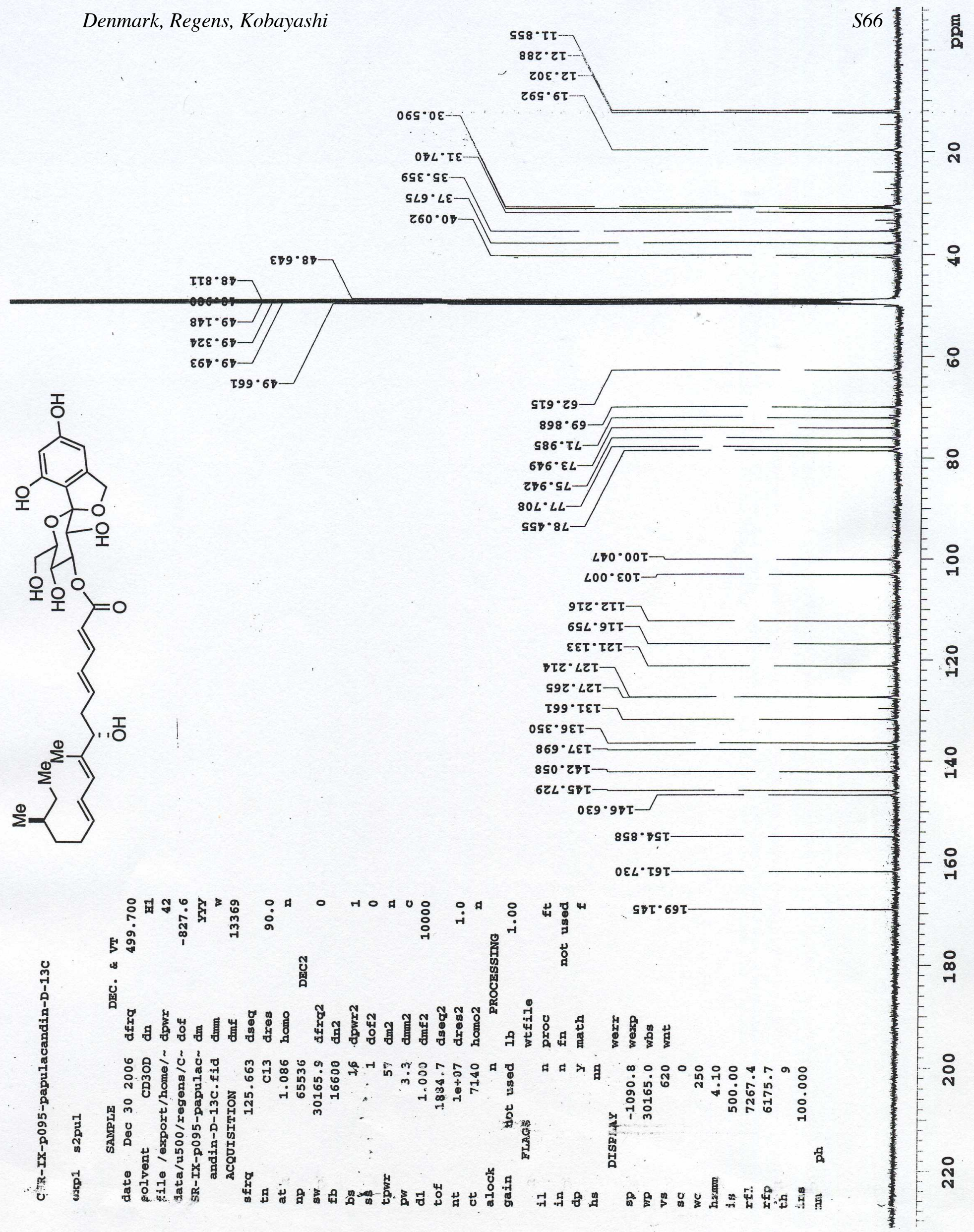